\title{
SHARP INTERFACE LIMIT FOR STOCHASTICALLY PERTURBED MASS CONSERVING ALLEN-CAHN EQUATION
}

\author{
BY TADAHISA FUNAKI ${ }^{1}$ AND SATOSHI YOKOYAMA \\ Waseda University
}

This paper studies the sharp interface limit for a mass conserving AllenCahn equation, added an external noise and derives a stochastically perturbed mass conserving mean curvature flow in the limit. The stochastic term destroys the precise conservation law, instead the total mass changes like a Brownian motion in time. For our equation, the comparison argument does not work, so that to study the limit we adopt the asymptotic expansion method, which extends that for deterministic equations used originally in de Mottoni and Schatzman [Interfaces Free Bound. 12 (2010) 527-549] for the nonconservative case and then in Chen et al. [Trans. Amer. Math. Soc. 347 (1995) 1533-1589] for the conservative case. Differently from the deterministic case, each term except the leading term appearing in the expansion of the solution in a small parameter $\varepsilon$ diverges as $\varepsilon$ tends to 0 , since our equation contains the noise which converges to a white noise and the products or the powers of the white noise diverge. To derive the error estimate for our asymptotic expansion, we need to establish the Schauder estimate for a diffusion operator with coefficients determined from higher order derivatives of the noise and their powers. We show that one can choose the noise sufficiently mild in such a manner that it converges to the white noise and at the same time its diverging speed is slow enough for establishing a necessary error estimate.

\section{CONTENTS}

1. Introduction and main results . . . . . . . . . . . . . . . . . . . . 561

1.1. Stochastic mass conserving Allen-Cahn equation and its background . . . . . . . . 561

1.2. Limit dynamics, conservation law and related results . . . . . . . . . . . . . . . . . . 562

1.3. Formulation of main results . . . . . . . . . . . . . . . . . . . . . . . 564

2. Asymptotic expansion of the solution of $(1.1) \ldots \ldots \ldots$. . . . . . . . . . . 567

2.1. Heuristic argument for the derivation of (1.4) . . . . . . . . . . . . . . . . . . 567

2.2. Signed distance from $\gamma_{t}$ and parametrization of $\gamma_{t} \ldots \ldots$. . . . . . . . . . . 569

2.3. Formal expansion of the solution $u^{\varepsilon} \ldots \ldots \ldots \ldots$. . . . . . . . . . . . . 572

2.4. Inductive scheme to determine coefficients . . . . . . . . . . . . . . . . . . . . . . . 574

2.5. Heuristic argument for the equation with spatially dependent noise . . . . . . . . . . 578

3. Approximate solutions and their estimates . . . . . . . . . . . . . . . . . . . . 579

3.1. Approximate solutions . . . . . . . . . . . . . . . . . . . . . . . 579

3.2. Derivation of $(2.43) \ldots \ldots \ldots \ldots \ldots \ldots \ldots$

Received October 2016; revised December 2017.

${ }^{1}$ Supported in part by the JSPS KAKENHI Grant Number (B) 26287014.

MSC2010 subject classifications. Primary 60H15; secondary 35K93, 74A50.

Key words and phrases. Sharp interface limit, Allen-Cahn equation, mean curvature flow, asymptotic expansion, mass conservation law, stochastic perturbation. 
3.3. Estimates for $u_{k}$ and $u_{k}^{ \pm} \ldots \ldots \ldots \ldots \ldots \ldots$

3.3.1. Bounds for spatial derivatives of $b, X_{0}, d$ and $\mathbf{S} \ldots \ldots \ldots 55$

3.3.2. Bounds for time derivatives of $X_{0}, \partial^{\mathbf{m}} X_{0}$ and $\mathbf{S} \ldots \ldots \ldots 86$

3.3.3. Schauder estimates for $h_{k} \ldots \ldots \ldots$. . . . . . . . . . 586

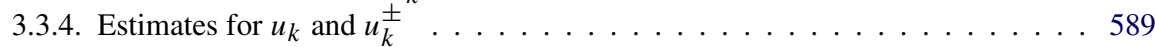

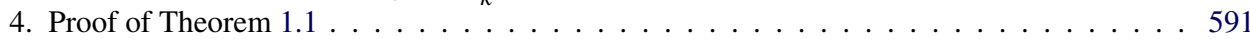

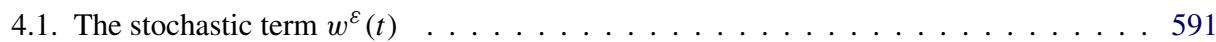

4.2. Error estimate . . . . . . . . . . . . . . . . . . . . . . . 593

4.3. The Allen-Cahn operator . . . . . . . . . . . . . . . . . . . . . . . . 593

4.4. Estimate for the difference between $u_{K}^{\varepsilon}$ and $u^{\varepsilon} \ldots \ldots \ldots \ldots \ldots 59 \ldots \ldots$

4.5. Proof of Theorem 1.1 . . . . . . . . . . . . . . . . . . . . . . . 598

5. Local existence and uniqueness for the limit dynamics (1.4) _ . . . . . . . . . . . 599

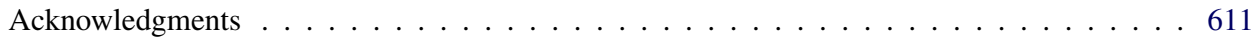

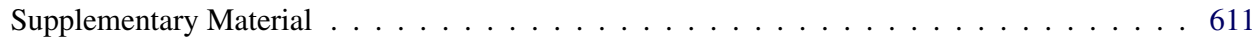

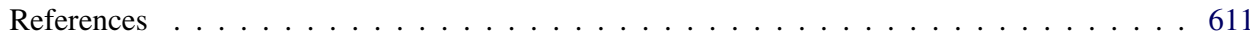

1. Introduction and main results. First, we introduce the stochastic mass conserving Allen-Cahn equation. Our goal is to discuss its sharp interface limit. The mass conservation law forces us to introduce a scaling for the noise different from the stochastic Allen-Cahn equation and prevents the comparison argument, which was useful for the stochastic Allen-Cahn equation. Our method is the asymptotic expansion, in which powers of derivatives of the noise appear repeatedly, and our main effort is devoted to controlling these diverging terms. The evolutional law of the limit hypersurface is described by the mass conserving mean curvature flow with a nonlocal multiplicative white noise term.

1.1. Stochastic mass conserving Allen-Cahn equation and its background. We consider the solution $u^{\varepsilon}=u^{\varepsilon}(t, x)$ of the following stochastic partial differential equation (1.1) in a bounded domain $D$ in $\mathbb{R}^{n}$ having a smooth boundary วD:

$$
\begin{cases}\frac{\partial u^{\varepsilon}}{\partial t}=\Delta u^{\varepsilon}+\varepsilon^{-2}\left(f\left(u^{\varepsilon}\right)-f_{D} f\left(u^{\varepsilon}\right)\right)+\alpha \dot{w}^{\varepsilon}(t) & \text { in } D \times \mathbb{R}_{+}, \\ \frac{\partial u^{\varepsilon}}{\partial v}=0 & \text { on } \partial \times \mathbb{R}_{+}, \\ u^{\varepsilon}(0, \cdot)=g^{\varepsilon}(\cdot) & \text { in } D,\end{cases}
$$

where $\varepsilon>0$ is a small parameter, $\alpha>0, v$ is the inward normal vector on $\partial D$, $\mathbb{R}_{+}=[0, \infty)$,

$$
f_{D} f\left(u^{\varepsilon}\right)=\frac{1}{|D|} \int_{D} f\left(u^{\varepsilon}(t, x)\right) d x,
$$

$g^{\varepsilon}$ are continuous functions having the property (1.12) stated below, or more precisely, satisfying the conditions (4.19)-(4.21). The noise $\dot{w}^{\varepsilon}(t)$ is the derivative of $w^{\varepsilon}(t) \equiv w^{\varepsilon}(t, \omega) \in C^{\infty}\left(\mathbb{R}_{+}\right)$in $t$ defined on a certain probability space $(\Omega, \mathcal{F}, P)$ 
such that $w^{\varepsilon}(t)$ converges to a one-dimensional standard Brownian motion $w(t)$ as $\varepsilon \downarrow 0$ in a suitable sense; see (1.8) and Assumption 1.1 below. We assume that the reaction term $f \in C^{\infty}(\mathbb{R})$ is bistable and satisfies the following three conditions:

$$
f( \pm 1)=0, \quad f^{\prime}( \pm 1)<0, \quad \int_{-1}^{1} f(u) d u=0
$$

(ii) $f$ has only three zeros \pm 1 and one another between \pm 1 ,

(iii) there exists $\bar{c}_{1}>0$ such that $f^{\prime}(u) \leq \bar{c}_{1}$ for every $u \in \mathbb{R}$.

The last equality in (i) is called the balance condition. A typical example is $f(u)=$ $u-u^{3}$. From (i) and (ii), it follows that there exists a unique increasing solution $m: \mathbb{R} \longrightarrow(-1,1)$ of

$$
m^{\prime \prime}+f(m)=0 \quad \text { on } \mathbb{R}, \quad m( \pm \infty)= \pm 1, \quad m(0)=0,
$$

and $m$ is called the traveling wave (or standing wave) solution. The function $m=$ $m(\rho), \rho \in \mathbb{R}$, satisfies

$$
\partial_{\rho}^{k}(m(\rho) \mp 1)=O\left(e^{-\zeta|\rho|}\right) \quad \text { as } \rho \rightarrow \pm \infty
$$

for $k=0,1,2, \ldots$, where $\zeta=\min \left\{\sqrt{-f^{\prime}(-1)}, \sqrt{-f^{\prime}(1)}\right\}>0$.

A mass conserving Allen-Cahn equation without noise, that is, (1.1) with $\alpha=0$, is introduced by Rubinstein and Sternberg [24]. Then the existence and uniqueness results for (1.1) with $\alpha=0$ are established by [5], [6], [19] and its sharp interface limit as $\varepsilon \downarrow 0$ is studied by Chen et al. [3]. Note that the general theory of partial differential equations [17] provides the well-posedness and the smoothness of the solutions for equation (1.1) having an additional smooth external force term $\alpha \dot{w}^{\varepsilon}(t)$. On the other hand, for a stochastic Allen-Cahn equation, that is, (1.1) without the averaged reaction term, the sharp interface limit is studied by Funaki [10], [11], Lions and Souganidis [22] and Weber [26]; see also [12], [13] which give a brief survey. The study of the sharp interface limit of the stochastic mass conserving Allen-Cahn equation (1.1) is a natural extension and combination of these two problems. Note that the noise term considered in [11], [26] was scaled as $\varepsilon^{-1} \alpha \dot{w}^{\varepsilon}(t)$. This is very different from $\alpha \dot{w}^{\varepsilon}(t)$ in (1.1). In other words, our dynamics are more sensitive to the noise. We will give a heuristic explanation for this difference in Section 2.1. Note also that the comparison argument used in [11], [26] does not work for equation (1.1). This is the main technical difference between our equation and the stochastic Allen-Cahn equation.

1.2. Limit dynamics, conservation law and related results. Our goal is to show that the solution $u^{\varepsilon}(t, x)$ of (1.1) converges as $\varepsilon \downarrow 0$ to $\chi_{\gamma_{t}}(x)$ with certain hypersurface $\gamma_{t}$ in $D$, where $\chi_{\gamma}(x)=+1$ or -1 according to the outside or inside of the hypersurface $\gamma$, if this holds for the initial data $g^{\varepsilon}$ with a certain $\gamma_{0}$, and the time evolution of $\gamma_{t}$ is governed by

$$
V=\kappa-f_{\gamma_{t}} \kappa+\frac{\alpha|D|}{2\left|\gamma_{t}\right|} \circ \dot{w}(t), \quad t \in[0, \sigma],
$$


up to a certain stopping time $\sigma>0$ (a.s.), where $V$ is the inward normal velocity of $\gamma_{t}, \kappa$ represents the mean curvature of $\gamma_{t}$ multiplied by $n-1,\left|\gamma_{t}\right|$ denotes the surface area of $\gamma_{t}, f_{\gamma_{t}} \kappa=\frac{1}{\left|\gamma_{t}\right|} \int_{\gamma_{t}} \kappa d \bar{s}, d \bar{s}$ stands for the volume element of $\gamma_{t}, \dot{w}(t)$ is the white noise process and $\circ$ means the Stratonovich stochastic integral. When $\alpha=0$, equation (1.4) coincides with the limit of the mass conserving Allen-Cahn equation studied in [3]. Note that, in [3], the sign of $V$ is taken opposite. When $\alpha=0$, the mass of the solution $u^{\varepsilon}$ of (1.1) is conserved, namely,

$$
\frac{1}{|D|} \int_{D} u^{\varepsilon}(t, x) d x=C,
$$

holds for some constant $C \in \mathbb{R}$. On the other hand, in the case where the fluctuation caused by $\alpha w^{\varepsilon}(t)$ is added, the rigid mass conservation law is destroyed and in place of (1.5), we have the conservation law in a stochastic sense

$$
\frac{1}{|D|} \int_{D} u^{\varepsilon}(t, x) d x=C+\alpha w^{\varepsilon}(t), \quad t \in \mathbb{R}_{+},
$$

which can be derived by integrating (1.1) over $D$ :

$$
\frac{1}{|D|} \int_{D}\left(\partial_{t} u^{\varepsilon}(t, x)-\alpha \dot{w}^{\varepsilon}(t)\right) d x=0 .
$$

Equation (1.6) implies that the total mass per volume behaves like a Brownian motion multiplied by $\alpha$ as $\varepsilon$ tends to 0 .

At least heuristically, the limit dynamics (1.4) is consistent to the conservation law (1.7) if the coefficient $c$ of the noise $\dot{w}(t)$ is given by $c=\frac{\alpha|D|}{2\left|\gamma_{t}\right|}$ as in (1.4). In fact, if $V>0$, the $(-1)$-phase transfers into $(+1)$-phase with speed $V$ per unit time. If $V<0$, an opposite transition occurs. Therefore, the rate of change of the total mass is given by $\partial_{t} \int_{D} u^{\varepsilon}(t, x) d x \approx 2 \int_{\gamma_{t}} V$. If $V$ is governed by the equation of the form of (1.4) with a coefficient $c$ in front of the noise $\dot{w}(t), 2 \int_{\gamma_{t}} V$ is equal to $2 c\left|\gamma_{t}\right| \dot{w}(t)$ and, for this to be consistent to (1.7) asymptotically as $\varepsilon \downarrow 0$, we would have $2 c\left|\gamma_{t}\right|=\alpha|D|$, and this implies $c=\frac{\alpha|D|}{2\left|\gamma_{t}\right|}$. See Section 2.1 for more detailed heuristic derivation of (1.4).

Equation (1.1) with $\alpha=0$ and without the averaged reaction term is called the Allen-Cahn equation. It is well known that the mean curvature flow $V=\kappa$ appears in the limit for this equation; cf., [1], [13]. For the stochastic Allen-Cahn equation, that is, (1.1) without the averaged reaction term and with the noise differently scaled as we mentioned above, the limit dynamics are given by $V=\kappa+\bar{\sigma} \alpha \dot{w}(t)$, where $\bar{\sigma}\left(\equiv c_{0}\right)$ is the inverse surface tension defined in (2.30) or in Section 2.1 below; see [11], (1.5). In this case, a simple additive noise appears in the limit, while in our case the limit dynamics (1.4) has a multiplicative noise and its coefficient contains a nonlocal term $\left|\gamma_{t}\right|^{-1}$. This is due to the effect of the conservation law. 
1.3. Formulation of main results. We take an integer $K$ satisfying $K>$ $\max (n+2,6)$ and fix it throughout the paper. This will be necessary for the proof of Theorem 4.9 later. Let $w^{\varepsilon}=w^{\varepsilon}(t) \equiv w^{\varepsilon}(t, \omega), 0<\varepsilon \leq 1, t \in \mathbb{R}_{+}, \omega \in \Omega$ be a family of $\left(\mathcal{F}_{t}\right)$-adapted stochastic processes defined on a probability space $(\Omega, \mathcal{F}, P)$ equipped with the filtration $\left(\mathcal{F}_{t}\right)_{t \geq 0}$, which satisfy that $w^{\varepsilon}(0)=0$, $w^{\varepsilon}(\cdot) \in C^{\infty}\left(\mathbb{R}_{+}\right)$in $t$ a.s. $\omega$ and

$$
\lim _{\varepsilon \downarrow 0}\left\|w^{\varepsilon}-w\right\|_{C^{\theta}([0, T])}=0 \quad \text { a.s., }
$$

for every $T>0$ and some $\theta \in\left(0, \frac{1}{2}\right)$, where $w(t)$ is an $\left(\mathcal{F}_{t}\right)$-Brownian motion satisfying $w(0)=0$ and

$$
\|v\|_{C^{\theta}([0, T])}=\sup _{t \in[0, T]}|v(t)|+\sup _{\substack{0 \leq s, t \leq T \\ s \neq t}} \frac{|v(t)-v(s)|}{|t-s|^{\theta}} .
$$

To prove our main theorem, we need two assumptions formulated as follows. The first assumption is on the speed of blowing up of the time derivatives of the noises, which is slow enough to control the diverging terms appearing in the expansion of $u^{\varepsilon}(t, x)$ in $\varepsilon$.

ASSUMPTION 1.1. For every $T>0$, there exists $H_{\varepsilon} \geq 1,0<\varepsilon \leq 1$, such that

$$
\begin{array}{rlrl}
\sup _{t \in[0, T], \omega \in \Omega}\left|\frac{d^{k}}{d t^{k}} w^{\varepsilon}(t, \omega)\right| & \leq H_{\varepsilon}, & & k=1,2, \ldots, n_{1}(K)+1, \\
\lim _{\varepsilon \downarrow 0} H_{\varepsilon} & =\infty, \quad & \lim _{\varepsilon \downarrow 0} \frac{H_{\varepsilon}^{2 n_{1}(K)}}{\log \log |\log \varepsilon|}=0,
\end{array}
$$

where $n_{1}(K) \in \mathbb{N}$ is the number determined from $K$ by Proposition 3.6 below.

Two examples of our mild noise $w^{\varepsilon}$ satisfying (1.8) and Assumption 1.1 will be given in Section 4.1.

The second assumption concerns the local existence and uniqueness for the limit dynamics (1.4) and the Wong-Zakai-type convergence theorem for the solutions as the noises converge.

ASSUMPTION 1.2. There exist stopping times $\sigma^{\varepsilon}$ and $\sigma$ such that $V^{\alpha \dot{w}^{\varepsilon}}$ (resp., $V$ ), the solution of (2.6) below with $v=\alpha \dot{w}^{\varepsilon}$ [resp. (1.4)], exists uniquely in $\left[0, \sigma^{\varepsilon}\right]$ (resp., $\left.[0, \sigma]\right)$. In addition, $\sigma^{\varepsilon}>0$ and $\sigma>0$ hold a.s. Furthermore, for every $T>0$ and $m \in \mathbb{N}$, the joint variable $\left(\sigma^{\varepsilon}, d^{\varepsilon}\left(t \wedge \sigma^{\varepsilon}\right)\right) \in \mathbb{R}_{+} \times C\left([0, T], C^{m}(\mathcal{O})\right)$ converges in this space to $(\sigma, d(t \wedge \sigma))$ as $\varepsilon \downarrow 0$ in a.s.-sense, where $d^{\varepsilon}(t)$ [resp., $d(t)]$ is the signed distance determined by the hypersurface $\gamma_{t}^{\alpha \dot{w}^{\varepsilon}}$ (resp., $\gamma_{t}$ ), which is negative inside $\gamma_{t}^{\alpha \dot{w}^{\varepsilon}}$ (resp., $\gamma_{t}$ ), and $\mathcal{O}$ is an open neighborhood of $\gamma_{0}$; see Theorem 1.1 and [26]. The signed distance $d(t)=d(t, x)$ is defined as $d(t, x)=\operatorname{sgn}(x) \inf _{y \in \gamma_{t}}|x-y|$ and $\operatorname{sgn}(x)=1$ or -1 if $x$ is outside of $\gamma_{t}$ or inside of $\gamma_{t}$, respectively. $d^{\varepsilon}(t, x)$ is similarly defined. 
Note that the smoothness of various constructions in the evolution of hypersurfaces is guaranteed by this assumption. We will show in Section 5 that Assumption 1.2 holds in the sense of law under a two-dimensional setting as long as the limit curve $\gamma_{t}$ stays convex. Applying Skorohod's representation theorem for joint variables $\left(w^{\varepsilon}, \sigma^{\varepsilon}, d^{\varepsilon}\left(t \wedge \sigma^{\varepsilon}\right)\right)$, by changing the probability space $(\Omega, \mathcal{F}, P)$ if necessary, one can realize the convergence in a.s.-sense as in (1.8) and Assumption 1.2. One of the reasons to formulate Assumption 1.2 in a general setting is that we have a hope to prove it in the future. Another reason is that one can clearly separate the methods in the theory of partial differential equations and those in stochastic analysis by formulating Assumption 1.2.

Assumption 1.2 implies uniform bounds on spatial derivatives of the distance functions in $\varepsilon>0$ locally in time; see Section 3.3.1. We also need bounds on $t$ derivatives of the hypersurface $\gamma_{t}^{\alpha \dot{w}^{\varepsilon}}$ by means of a certain norm of the noise $\dot{w}^{\varepsilon}$. This will be formulated precisely as Assumption 3.1 in Section 3.3.2 and shown under two-dimensional settings in Section 5.

The aim of this paper is to prove the following theorem.

THEOREM 1.1. Let $\gamma_{0}$ be a smooth hypersurface in $D$ without boundary with finitely many connected components and it has the form $\gamma_{0}=\partial D_{0}$ with a smooth domain $D_{0}$ such that $\bar{D}_{0} \subset D$. Suppose that a local solution $\Gamma=\bigcup_{0 \leq t<\sigma}\left(\gamma_{t} \times\{t\}\right)$ of (1.4) up to the stopping time $\sigma>0$ (a.s.) satisfying $\gamma_{t} \subset D$ for all $t \in[0, \sigma]$ uniquely exists (a.s.). Furthermore, let us assume three Assumptions 1.1, 1.2 and 3.1. Then one can find a family of continuous functions $\left\{g^{\varepsilon}(\cdot)\right\}_{\varepsilon \in(0,1]}$ satisfying

$$
\lim _{\varepsilon \downarrow 0} g^{\varepsilon}(x)=\chi_{\gamma_{0}},
$$

such that $u^{\varepsilon}\left(t \wedge \sigma^{\varepsilon} \wedge \tau, \cdot\right)$ converges to $\chi_{\gamma_{t \wedge \sigma \wedge \tau}}(\cdot)$ in $C\left(\mathbb{R}_{+}, L^{2}(D)\right)$ as $\varepsilon \downarrow 0$ in a.s.-sense, where $u^{\varepsilon}$ is the solution of (1.1) with initial value $g^{\varepsilon}$ and $\tau=\tau(\omega)>0$ is that given below Assumption 3.1.

If Assumption 1.2 holds in the sense of law, by the observation we gave above, Theorem 1.1 holds also in the sense of law. More precise conditions for $g^{\varepsilon}$ are formulated in (4.19)-(4.21).

The right-hand side of the first equation in (1.1) contains the averaged reaction term, and hence, as we already pointed out, one cannot directly apply the comparison principle to estimate its solution. This is a difference from the stochastic Allen-Cahn equation treated in [11], [26]. Our method for the proof of Theorem 1.1 is an extension of the asymptotic expansion used originally in de Mottoni and Schatzman [4] and then in [3].

Recall that $w(t)$ is not in $C^{1}$-class in $t$ and, therefore, for any smooth sequence $\left\{w^{\varepsilon}(t)\right\}_{0<\varepsilon \leq 1}$ converging toward $w(t)$, the products or the powers of its time derivative $\left\{\dot{w}^{\varepsilon}(t)\right\}_{0<\varepsilon \leq 1}$ and higher order time derivatives always diverge as 
$\varepsilon \downarrow 0$. Due to this, each term in inner and outer solutions constructed by the asymptotic expansion except its leading term explodes as $\varepsilon \downarrow 0$ because it contains some powers of the time derivatives of $w^{\varepsilon}$. [Here, the inner solution is defined through the expansion of $u^{\varepsilon}$ near the interface in a stretched (microscopic) spatial variable $\rho \in \mathbb{R}$, which is introduced to relax the sharp transition of $u^{\varepsilon}$ near the interface, rather than the original (macroscopic) variable $x \in D$, while the outer solution determines the boundary condition of the inner solution at $\rho= \pm \infty$; see Section 2.3 for more details. The meaning of the outer solution becomes clear in Section 2.1.] However, by choosing the sequence $\dot{w}^{\varepsilon}(t)$ in a suitable manner that its divergent speed is slow enough as in Assumption 1.1, one can control the diverging terms. Indeed, the speed of divergence of each $k$ th inner and outer solutions having prefactor $\varepsilon^{k}$ can be controlled once we can make the divergent speed of the powers of time derivatives of $w^{\varepsilon}(t)$ slower than $\varepsilon^{-k}$. This is one of the key points in the proof of Theorem 1.1.

The paper is organized as follows. In Section 2, we first give a heuristic derivation of the evolutional law (1.4) of $\gamma_{t}$, as a result of the combination of (2.4) and (2.30). Then we introduce the asymptotic expansion of the solution $u^{\varepsilon}$ of equation (1.1) in $\varepsilon$ in detail. It turns out that one needs to analyze the asymptotic expansion up to the $K$ th order term with $K>\max (n+2,6)$; cf. Lemma 2 of [3] and Theorem 4.9 below. We briefly touch the stochastic mass conserving Allen-Cahn equation with a spatially dependent noise with vanishing spatial average, and compare the scaling of the noise to our case. In Section 3, we define an approximate solution and show the estimates on each term in the asymptotic expansion. This is accomplished by carefully studying the Schauder estimate for a diffusion operator with diverging coefficients. In particular, we need to study how the constants in the Schauder estimate depend on the coefficients, especially the norm of the noise $\dot{w}^{\varepsilon}$. As a result, we find out that Assumption 1.1 for $\dot{w}^{\varepsilon}$ is enough to control the expansion of $u^{\varepsilon}$. Then in Section 4, we give the proof of Theorem 1.1. In Section 5, we discuss the stochastic partial differential equation (SPDE) corresponding to (1.4) under the situation that $n=2$ and the interfaces stay convex and show that the SPDE corresponding to (1.4) has a unique local solution in such case. Assumption 1.2 in law sense and Assumption 3.1 are shown in this situation.

Proofs of all the lemmas in Sections 3-5, with some exceptions, are deferred to the Supplementary Materials [14].

REMARK 1.1. Weber [26] established the sharp interface limit for the stochastic Allen-Cahn equation in a.s.-sense with the choice of $\dot{w}^{\varepsilon}(t)$ such as the first example given in Section 4.1. In his argument, it was essential that the SPDE describing the dynamics of the limit hypersurface $\gamma_{t}$ in terms of the signed distance $d$ has an additive noise. This is not the case for our limit dynamics (1.4) and his argument does not work or at least requires substantial modifications for showing Assumption 1.2 under a more general setting than we discuss in Section 5. 


\section{Asymptotic expansion of the solution of (1.1).}

2.1. Heuristic argument for the derivation of (1.4). Before starting the proof of Theorem 1.1, it might be worthy to give a heuristic derivation of the evolutional law (1.4) of the limit hypersurface $\gamma_{t}$ from the stochastically perturbed mass conserving Allen-Cahn equation (1.1).

Note that the scaling for the noise term in (1.1) is essentially different from that for the stochastic Allen-Cahn equation. In fact, without the term $-f_{D} f\left(u^{\varepsilon}\right)$ in (1.1), the proper scaling for the noise term was $\varepsilon^{-1} \alpha \dot{w}^{\varepsilon}(t)$ rather than $\alpha \dot{w}^{\varepsilon}(t)$; see [11]. We give a heuristic argument to explain the reason for this difference; see also Section 2.5 for a spatially dependent noise. In particular, we will see that the averaged reaction term behaves as $f_{D} f\left(u^{\varepsilon}\right)=O(\varepsilon)$ as $\varepsilon \downarrow 0$ so that $\varepsilon^{-2}-$ $\int_{D} f\left(u^{\varepsilon}\right) \approx \varepsilon^{-1} \lambda_{0}(t)$ with a certain $\lambda_{0}(t)$, and the evolution of $\lambda_{0}(t)$ is governed by the noise $\alpha \dot{w}^{\varepsilon}(t)$ of $O(1)$.

Our basic ansatz is that $u^{\varepsilon}(t, x) \approx \pm 1$ as $\varepsilon \downarrow 0$. We actually assume this at $t=0$ as in (1.12). This implies $f\left(u^{\varepsilon}\right) \approx 0$ so that $a^{\varepsilon}(t):=f_{D} f\left(u^{\varepsilon}\right)$ should be small; see Remark 2.1 below. Conversely, if $a^{\varepsilon}(t)$ is small, the main term of the reaction term becomes $\varepsilon^{-2} f\left(u^{\varepsilon}\right)$ so that the solution $u^{\varepsilon}$ is pushed toward \pm 1 and we can expect our ansatz to be true. Anyway, this observation suggests that, instead of $m$ defined by (1.2), it might be better to consider the perturbed traveling wave solutions with the reaction term $f$ replaced by $f-a^{\varepsilon}(t)+\varepsilon^{2} v(t)$ and $v(t)=\alpha \dot{w}^{\varepsilon}(t)$. We denote $v(t)$ for $\alpha \dot{w}^{\varepsilon}(t)$, since this term could be regarded as $O(1)$ as $\varepsilon \downarrow 0$; see (2.5) below. More precisely, for $a \in \mathbb{R}$ with small $|a|$, define the traveling wave solution $m=m(\rho ; a), \rho \in \mathbb{R}$ and its speed $c=c(a)$ by

$$
\left\{\begin{array}{l}
m^{\prime \prime}(\rho)+c m^{\prime}(\rho)+\{f(m(\rho))-a\}=0, \quad \rho \in \mathbb{R}, \\
m( \pm \infty)=m_{ \pm}^{*}, \quad m(0)=0,
\end{array}\right.
$$

where $m_{ \pm}^{*} \equiv m_{ \pm}^{*}(a)= \pm 1+O(a)(a \rightarrow 0)$ are solutions of $f\left(m_{ \pm}^{*}\right)-a=0$. It is easy to see that $c_{0}:=c^{\prime}(0)=2 / \int_{\mathbb{R}} m^{\prime}(\rho)^{2} d \rho$; see below. Another expression of $c_{0}$ is also known: $c_{0}=\frac{\sqrt{2}}{\int_{-1}^{1} \sqrt{V(u)} d u}$ where $V(u)=\int_{u}^{1} f(v) d v$; see [11].

Our guess for the behavior of the solution $u^{\varepsilon}$ of (1.1) is the following:

$$
u^{\varepsilon}(t, x)=m\left(\frac{d(t, x)}{\varepsilon} ; a^{\varepsilon}(t)-\varepsilon^{2} v(t)\right)+O(\varepsilon),
$$

as $\varepsilon \downarrow 0$, where $d(t, x)$ is the signed distance between $x \in D$ and the limit hypersurface $\gamma_{t}$. Then, denoting $m\left(\cdot ; a^{\varepsilon}(t)-\varepsilon^{2} v(t)\right)$ simply by $m$, we have

$$
\begin{aligned}
0= & \int_{D}\left\{\Delta u^{\varepsilon}+\frac{1}{\varepsilon^{2}}\left(f\left(u^{\varepsilon}\right)-f_{D} f\left(u^{\varepsilon}\right)\right)\right\} d x \\
\approx & \frac{1}{\varepsilon^{2}} \int_{D}\left\{m^{\prime \prime}\left(\frac{d}{\varepsilon}\right)+\varepsilon m^{\prime}\left(\frac{d}{\varepsilon}\right) \Delta d+f\left(m\left(\frac{d}{\varepsilon}\right)\right)-a^{\varepsilon}(t)+\varepsilon^{2} v(t)\right\} d x \\
& -|D| v(t)
\end{aligned}
$$




$$
\begin{aligned}
& =\frac{1}{\varepsilon^{2}} \int_{D}\left\{-c\left(a^{\varepsilon}(t)-\varepsilon^{2} v(t)\right) m^{\prime}\left(\frac{d}{\varepsilon}\right)+\varepsilon m^{\prime}\left(\frac{d}{\varepsilon}\right) \Delta d\right\} d x-|D| v(t) \\
& \approx \frac{1}{\varepsilon^{2}} \int_{D} m^{\prime}\left(\frac{d}{\varepsilon}\right)\left\{-c_{0} a^{\varepsilon}(t)+\varepsilon \Delta d\right\} d x-|D| v(t) \\
& \approx \frac{1}{\varepsilon^{2}} \int_{\mathbb{R}} \int_{\gamma_{t}} m^{\prime}\left(\frac{r}{\varepsilon}\right)\left\{-c_{0} a^{\varepsilon}(t)+\varepsilon \kappa(t, \bar{s})+\varepsilon r b(t, \bar{s})\right\} d r d \bar{s}-|D| v(t) \\
& \approx \int_{\mathbb{R}} \int_{\gamma_{t}} m^{\prime}(\rho)\left\{-\frac{1}{\varepsilon} c_{0} a^{\varepsilon}(t)+\kappa(t, \bar{s})+\varepsilon \rho b(t, \bar{s})\right\} d \rho d \bar{s}-|D| v(t) \\
& =\left(m_{+}^{*}-m_{-}^{*}\right) \int_{\gamma_{t}}\left\{-c_{0} \frac{a^{\varepsilon}(t)}{\varepsilon}+\kappa(t, \bar{s})\right\} d \bar{s}-|D| v(t)+O(\varepsilon) .
\end{aligned}
$$

Here, the first line is a consequence of $\int_{D} \Delta u^{\varepsilon} d x=0$ which holds under the Neumann condition at $\partial D$, we apply (2.2) for the second line noting that $\Delta u^{\varepsilon} \approx$ $\Delta\left(m\left(\frac{d}{\varepsilon}\right)\right)=\frac{1}{\varepsilon^{2}} m^{\prime \prime}\left(\frac{d}{\varepsilon}\right)|\nabla d|^{2}+\frac{1}{\varepsilon} m^{\prime}\left(\frac{d}{\varepsilon}\right) \Delta d$ and $|\nabla d|=1$ at least near $\gamma_{t}$, the third line follows from (2.1) with $a=a^{\varepsilon}(t)-\varepsilon^{2} v(t)$, the fourth line by $c\left(a^{\varepsilon}-\varepsilon^{2} v\right)=$ $c_{0} a^{\varepsilon}+O\left(\left(a^{\varepsilon}\right)^{2}\right)+O\left(\varepsilon^{2}\right)$ and $c(0)=0$, the fifth line from (30), (40) of [3], that is, $\Delta d=\kappa(t, \bar{s})+r b(t, \bar{s})+O(\varepsilon)$ with $b(t, \bar{s})=-\nabla d \cdot \nabla \Delta d$ for $\bar{s} \in \gamma_{t}$ and the sixth line follows by the change of variables $r=\varepsilon \rho$. The above computation implies that $a^{\varepsilon}(t)$ should be of order $O(\varepsilon)$ and, defining $\lambda_{0}(t)$ as

$$
\frac{1}{\varepsilon} a^{\varepsilon}(t) \equiv \frac{1}{\varepsilon} f_{D} f\left(u^{\varepsilon}(t, \cdot)\right)=\lambda_{0}(t)+O(\varepsilon),
$$

since $m_{ \pm}^{*}= \pm 1+O\left(a^{\varepsilon}(t)-\varepsilon^{2} v(t)\right)= \pm 1+O(\varepsilon)$, we obtain

$$
2 c_{0} \lambda_{0}(t)\left|\gamma_{t}\right|=2 \int_{\gamma_{t}} \kappa d \bar{s}-|D| v(t)
$$

in the limit $\varepsilon \downarrow 0$.

It will be clear that this condition is necessary for the first term of (3.13) to vanish and used in (2.29) to determine $\lambda_{0}(t)$; note that $c_{0}=\bar{\sigma}:=2\left(\int_{\mathbb{R}} m^{\prime}(\rho)^{2} d \rho\right)^{-1}$ holds as we will see below, where $\bar{\sigma}$ is called the inverse surface tension. In particular, (2.2) and (2.3) suggest

$$
u^{\varepsilon}(t, x) \underset{x=(\bar{s}, r), r \rightarrow \pm \infty}{\sim} m_{ \pm}^{*}\left(a^{\varepsilon}(t)-\varepsilon^{2} v(t)\right) \sim \pm 1+\varepsilon \frac{\lambda_{0}(t)}{f^{\prime}( \pm 1)},
$$

since $0=f\left(m_{ \pm}^{*}\right)-a \sim f^{\prime}( \pm 1)\left(m_{ \pm}^{*} \mp 1\right)-a$, which implies $m_{ \pm}^{*} \sim \pm 1+\frac{a}{f^{\prime}( \pm 1)}$. This exactly coincides with the formula (2.34) for the asymptotic behavior of the outer solutions.

Once (2.4) is obtained, (1.4) could be derived from

$$
V=\kappa-\bar{\sigma} \lambda_{0}(t) \quad \text { on } \gamma_{t},
$$

which is obtained as a solvability condition for $u_{0}$ appearing in the expansion of $u^{\varepsilon}$; see (2.30) below. Later, we will consider the expansion of $u^{\varepsilon}$ based on $m\left(\frac{d(t, x)}{\varepsilon} ; 0\right)$ 
with $a=0$ rather than that introduced in (2.2), since the leading orders are the same.

We finally comment on the identity $c_{0}=\bar{\sigma}$. The smoothness of $c(a)$ in $a$ is shown in the Appendix of [7]. We compute for $m=m(\cdot ; a)$ as

$$
\begin{aligned}
0 & =-\int_{\mathbb{R}} \frac{1}{2}\left\{\left(m^{\prime}\right)^{2}\right\}^{\prime} d \rho=-\int_{\mathbb{R}} m^{\prime \prime} \cdot m^{\prime} d \rho \\
& =\int_{\mathbb{R}}\left\{c(a) m^{\prime}+f(m)-a\right\} m^{\prime} d \rho \\
& =c(a) \int_{\mathbb{R}}\left(m^{\prime}\right)^{2} d \rho+\int_{m_{-}^{*}}^{m_{+}^{*}} f(s) d s-a\left(m_{+}^{*}-m_{-}^{*}\right) .
\end{aligned}
$$

Take the derivative of both sides in $a$ and set $a=0$ noting that $m^{\prime}=m^{\prime}(\rho ; a)$ and $m_{ \pm}^{*}=m_{ \pm}^{*}(a)$. This leads to the identity $c_{0}=\bar{\sigma}$ since $m_{ \pm}^{*}(a)= \pm 1+O(a)$ as $a \rightarrow 0$.

REMARK 2.1. If the condition (1.12) does not hold for the initial data $g^{\varepsilon}$, $a^{\varepsilon}(0)$ is not small in general. In this case, $a^{\varepsilon}(t)$ may not be small as well. This means that the reaction term $f(u)-a^{\varepsilon}(t)$ does not satisfy the balance condition, that is, the last equality in the condition (i) for $f$. Thus, the situation is more close to that of Gärtner [15] and others discussed at least in the nonrandom case; see Section 4.1 of [13] and also Hilhorst et al. [18]. In particular, the proper time scale and the limit dynamics should be totally different from ours.

2.2. Signed distance from $\gamma_{t}$ and parametrization of $\gamma_{t}$. Let us start more precise discussions. The expansion of the solution $u^{\varepsilon}(t, x)$ of (1.1) in $\varepsilon$ will be given only in $\varepsilon$ appearing in the reaction term and not that in the noise term. To make this clear, we consider the following equation with an external force $v(t)$, which is deterministic (nonrandom) such that $v \in C^{\infty}\left(\mathbb{R}_{+}\right)$:

$$
\begin{cases}\frac{\partial u^{\varepsilon}}{\partial t}=\Delta u^{\varepsilon}+\varepsilon^{-2}\left(f\left(u^{\varepsilon}\right)-f_{D} f\left(u^{\varepsilon}\right)\right)+v(t) & \text { in } D \times \mathbb{R}_{+}, \\ \frac{\partial u^{\varepsilon}}{\partial v}=0 & \text { on } \partial D \times \mathbb{R}_{+}, \\ u^{\varepsilon}(\cdot, 0)=g^{\varepsilon}(\cdot) & \text { in } D .\end{cases}
$$

Needless to say, the solution of (1.1) is the same as that of (2.5) with $v=\alpha \dot{w}^{\varepsilon}$. In addition, we consider the hypersurface $\left\{\gamma_{t}^{v}\right\}$ whose evolution is governed by

$$
V^{v}=\kappa-f_{\gamma_{t}^{v}} \kappa+\frac{|D|}{2\left|\gamma_{t}^{v}\right|} v(t),
$$

where $V^{v}$ is the inward normal velocity of $\gamma_{t}^{v}$. From Assumption 1.2, (2.6) has a unique solution for $t \leq T^{v}$ with some $T^{v}>0$. Under these settings, we will first 
expand the solution $u^{\varepsilon}=u^{\varepsilon, v}$ of (2.5) in $\varepsilon$ based on the solution $\gamma_{t}=\gamma_{t}^{v}$ of (2.6). Next, we will estimate each term appearing in the expansion by a suitable norm of $v$; see Proposition 3.6 and Lemma 4.3. Finally, in Sections 4.2-4.5, we will apply these lemmas taking $v(t)=\alpha \dot{w}^{\varepsilon}(t)$.

In Sections 2.2 and 2.3, we copy several notation and computations from [3]. Let $d=d^{v}(t, x)$ be the signed distance of $x \in D$ to the hypersurface $\gamma_{t}$, which is negative inside $\gamma_{t}$; cf. Bellettini [1], Chapter 1. To parametrize the hypersurface $\gamma_{t}$ on a fixed reference manifold, we go along with [1], Chapter 16 as follows. Let $\mathcal{S} \subset \mathbb{R}^{n}$ be an oriented compact smooth $(n-1)$-dimensional submanifold without boundary and with finitely many connected components being smoothly embedded in $\mathbb{R}^{n}$. For each $s=\left(s^{l}\right)_{l=1}^{n} \in \mathcal{S}$, except some singular points, $s^{n}$ is represented by other coordinates such that $s^{n}=s^{n}\left(s^{1}, \ldots, s^{n-1}\right)$, and thus we can take $s=$ $\left(s^{l}\right)_{l=1}^{n-1}$ as a local coordinate of $\mathcal{S}$. Near singular points for $s^{n}$, one may take other coordinates, for example, $\left(s^{l}\right)_{l=2}^{n}$, but we denote it by $s=\left(s^{l}\right)_{l=1}^{n-1}$ for simplicity. Then $\left\{\frac{\partial}{\partial s^{l}}\right\}_{l=1}^{n-1}$ forms a basis of the tangent space $T_{S} \mathcal{S}$ at $s \in \mathcal{S}$. We parametrize $\gamma_{t}, t \in[0, T]$ as $x=X_{0}(t, s)$ by $s=\left(s^{l}\right)_{l=1}^{n-1} \in \mathcal{S}$ such that $X_{0} \in C^{\infty}([0, T] \times$ $\left.\mathcal{S}, \mathbb{R}^{n}\right)$ and the map $X_{0}(t, \cdot): \mathcal{S} \rightarrow \gamma_{t}$ is homeomorphic for every $t \in[0, T]$. See Remark 2.2 below to see that this is possible. In particular, $\left(\frac{\partial X_{0}(t, s)}{\partial s^{1}}, \ldots, \frac{\partial X_{0}(t, s)}{\partial s^{n-1}}\right)$ forms a basis of the tangent space to $\gamma_{t}$ at $x=X_{0}(t, s)$ for each $s \in \mathcal{S}$.

We denote by $\mathbf{n}(t, s)$ the unit outer normal vector on $\gamma_{t}$ so that

$$
\mathbf{n}(t, s)=\nabla d\left(t, X_{0}(t, s)\right) .
$$

We define the Jacobian of the map $X_{0}(t, \cdot)$ as

$$
J^{0}(t, s)=\operatorname{det}\left[\mathbf{n}(t, s), \partial_{s^{1}} X_{0}(t, s), \ldots, \partial_{s^{n-1}} X_{0}(t, s)\right] .
$$

REMARK 2.2. Bellettini ([1], page 251) introduces the parametrization $\varphi_{\varepsilon}(t, s)$ of the hypersurface $\tilde{\gamma}_{t}^{\varepsilon}$ determined by (2.18) below. Our parametrization $X_{0}(t, s)$ of $\gamma_{t}$ by $\mathcal{S}$ is similar and corresponds to $\varphi_{0}(t, s)$ [i.e., $\varphi_{\varepsilon}(t, s)$ with $\varepsilon=0$ ] in [1]; see especially Remark 3.6 and Definition 16.1 of [1]. Chen et al. [3], (31) considers the parametrization by $U$ instead of our $\mathcal{S}$ satisfying

$$
J^{0}(t, s)=1,
$$

but under this parametrization the set $U$ changes in $t$. To avoid this inconvenience, we follow [1].

Let $\delta>0$ be small enough such that the signed distance function $d(t, x)$ from $\gamma_{t}$ is smooth in the $3 \delta$-neighborhood of $\gamma_{t}=\gamma_{t}^{v}$ (recall Assumption 1.2) and the distance between $\gamma_{t}$ and $\partial D$ is larger than $3 \delta$ for every $t \in\left[0, T^{v}\right]$. A local coordinate $(r, s) \in(-3 \delta, 3 \delta) \times \mathcal{S}$ of $x$ in a tubular neighborhood of $\gamma_{t}$ is defined by

$$
x=X_{0}(t, s)+r \mathbf{n}(t, s)=: X(t, r, s) .
$$


In other words, $X_{0}(t, s)$ is the point on $\gamma_{t}$ closest from $x$. Then its inverse function on a tubular neighborhood of $\gamma_{t}$ is given by

$$
r=d(t, x), s=\mathbf{S}(t, x)=\left(S^{1}(t, x), \ldots, S^{n-1}(t, x)\right) .
$$

In particular, since $r=d\left(t, X_{0}(t, s)+r \mathbf{n}(t, s)\right)$, by differentiating this in $r$, we have

$$
\nabla d\left(t, X_{0}(t, s)+r \mathbf{n}(t, s)\right) \cdot \mathbf{n}(t, s)=1, \quad r \in(-3 \delta, 3 \delta) .
$$

Since $|\nabla d(t, x)|=1$ for $x$ close to $\gamma_{t}$, this implies

$$
\nabla d\left(t, X_{0}(t, s)+r \mathbf{n}(t, s)\right)=\mathbf{n}(t, s), \quad r \in(-3 \delta, 3 \delta) .
$$

Changing coordinates from $(t, x)$ to $(t, r, s)$ for a function $\phi=\phi(t, x)$, we associate another function $\tilde{\phi}=\tilde{\phi}(t, r, s)$ as

$$
\tilde{\phi}(t, r, s)=\phi\left(t, X_{0}(t, s)+r \mathbf{n}(t, s)\right),
$$

or equivalently

$$
\phi(t, x)=\tilde{\phi}(t, d(t, x), \mathbf{S}(t, x)) .
$$

Let $V(t, s)$ be the inward normal velocity of the interface $\gamma_{t}$ at $X_{0}(t, s)$,

$$
V(t, s)=-\partial_{t} X_{0}(t, s) \cdot \mathbf{n}(t, s) .
$$

From $(2.7)$ and $d\left(t, X_{0}(t, s)\right)=0$, we see that

$$
V(t, s)=\partial_{t} d\left(t, X_{0}(t, s)\right) .
$$

Then we have

$$
\begin{aligned}
& \partial_{t} \phi(t, x)=\left(V \partial_{r}+\partial_{t}^{\Gamma}\right) \tilde{\phi}(t, d(t, x), \mathbf{S}(t, x)), \\
& \nabla \phi(t, x)=\left(\mathbf{n}(t, \mathbf{S}(t, x)) \partial_{r}+\nabla^{\Gamma}\right) \tilde{\phi}(t, d(t, x), \mathbf{S}(t, x)), \\
& \Delta \phi(t, x)=\left(\partial_{r}^{2}+\Delta d(t, x) \partial_{r}+\Delta^{\Gamma}\right) \tilde{\phi}(t, d(t, x), \mathbf{S}(t, x)),
\end{aligned}
$$

where the superscripts $\Gamma$ mean the derivatives tangential to the hypersurface $\gamma^{v}$ seen under the coordinate $s \in \mathcal{S}$ :

$$
\begin{aligned}
& \partial_{t}^{\Gamma} \tilde{\phi}=\left(\partial_{t}+\sum_{i=1}^{n-1} S_{t}^{i} \partial_{s^{i}}\right) \tilde{\phi}, \\
& \nabla^{\Gamma} \tilde{\phi}=\left(\sum_{i=1}^{n-1} \partial_{1} S^{i} \partial_{s^{i}}, \ldots, \sum_{i=1}^{n-1} \partial_{n} S^{i} \partial_{s^{i}}\right) \tilde{\phi}, \\
& \Delta^{\Gamma} \tilde{\phi}=\left(\sum_{i=1}^{n-1} \Delta S^{i} \partial_{s^{i}}+\sum_{i, j=1}^{n-1} \nabla S^{i} \cdot \nabla S^{j} \partial_{s^{i} s^{j}}^{2}\right) \tilde{\phi},
\end{aligned}
$$


see (33) in [3] recalling that the sign of $V$ is opposite. We denote by $\kappa_{1}, \ldots, \kappa_{n-1}, 0$ the eigenvalues of the Hessian $D_{x}^{2} d(t, x)$ with corresponding normalized eigenvectors $\tau_{1}, \ldots, \tau_{n-1}, \nabla d$; note that $D_{x}^{2} d(t, x) \nabla d(t, x)=0$ so that 0 is an eigenvalue of the Hessian and $\nabla d$ is the corresponding eigenvector; cf. (28) in [3]. Set

$$
\kappa(t, s):=(n-1) \bar{\kappa}_{\gamma_{t}}=\sum_{i=1}^{n-1} \kappa_{i}=\Delta d\left(t, X_{0}(t, s)\right),
$$

where $\bar{\kappa}_{\gamma_{t}}$ is the mean curvature of $\gamma_{t}$ at $x=X_{0}(t, s)$. We denote

$$
b(t, s):=-\left.\nabla d \cdot \nabla \Delta d(t, x)\right|_{x=X_{0}(t, s)}=\sum_{i=1}^{n-1} \kappa_{i}^{2} .
$$

2.3. Formal expansion of the solution $u^{\varepsilon}$. In this subsection, we briefly recall in our setting the method of construction of inner and outer solutions given in [3]. Equation (2.5) is expressed as

$$
0=f\left(u^{\varepsilon}(t, x)\right)+\varepsilon^{2}\left(-\partial_{t} u^{\varepsilon}(t, x)+\Delta u^{\varepsilon}(t, x)+v(t)\right)-\varepsilon \lambda_{\varepsilon}(t),
$$

where $\lambda_{\varepsilon}(t):=\varepsilon^{-1} a^{\varepsilon}(t)$ is given in (2.3), that is,

$$
\lambda_{\varepsilon}(t):=\varepsilon^{-1} f_{D} f\left(u^{\varepsilon}(t, \cdot)\right) .
$$

Note that (2.15) combined with the conservation law

$$
\partial_{t} \int_{D} u^{\varepsilon}(t, x) d x=|D| v(t)
$$

implies (2.16) and, therefore, (2.5).

Our first guess for the behavior of the solution $u^{\varepsilon}$ of (2.5) was given by (2.2). The term $m$ in the right-hand side of (2.2) attains zeros exactly at $\gamma_{t}=\{d(t, x)=$ $0\}$. However, with the error term $O(\varepsilon)$, it is reasonable to expect the zeros $Z_{t}^{\varepsilon} \equiv$ $\left\{x \in D \mid u^{\varepsilon}(t, x)=0\right\}$ of $u^{\varepsilon}$ is close to $\gamma_{t}$ and has a distance of $O(\varepsilon)$ from $\gamma_{t}$. This suggests to introduce a new variable $h_{\varepsilon}(t, s)$ and a set

$$
\tilde{\gamma}_{t}^{\varepsilon}=\left\{X(t, r, s) \mid r=\varepsilon h_{\varepsilon}(t, s), s \in \mathcal{S}\right\},
$$

which, we expect, is almost $Z_{t}^{\varepsilon}$; cf. (35) of [3]. We will expand $u^{\varepsilon}, \lambda_{\varepsilon}$ and $h_{\varepsilon}$ in $\varepsilon$; see (2.24) and (2.25) below. In fact, $h_{\varepsilon}(t, s)$ will be determined in such a way that the leading term in the expansion of $\tilde{u}^{\varepsilon}(t, \rho, s)$, which is defined from $u^{\varepsilon}(t, x)$ by shifting by $\varepsilon h^{\varepsilon}$, is exactly $m(\rho)$ as we will see in (2.24).

Let us define the stretched variable $\rho=\rho^{\varepsilon}(t, x)$ as

$$
\rho^{\varepsilon}(t, x)=\frac{d(t, x)-\varepsilon h_{\varepsilon}(t, \mathbf{S}(t, x))}{\varepsilon},
$$

which is nearly the distance between $x$ and $\tilde{\gamma}_{t}^{\varepsilon}$ (more precisely, the distance between $x$ and $\gamma_{t}$ minus that between $\tilde{\gamma}_{t}^{\varepsilon}$ and $\gamma_{t}$ measured at the projected point of 
$x$ onto $\gamma_{t}$ ) divided by $\varepsilon>0$; see below for the reason to introduce the stretched (microscopic) variable. Then the variables $(t, x)$ and $(t, \rho, s)$ are related by

$$
\begin{aligned}
x & =\hat{X}(t, \rho, s)=X\left(t, \varepsilon\left(\rho+h_{\varepsilon}(t, s)\right), s\right) \\
& =X_{0}(t, s)+\varepsilon\left(\rho+h_{\varepsilon}(t, s)\right) \mathbf{n}(t, s) .
\end{aligned}
$$

Furthermore, its Jacobian $J^{\varepsilon}(t, \rho, s)$ defined by $d x=\varepsilon J^{\varepsilon}(t, \rho, s) d \rho d s$ is written as

$$
J^{\varepsilon}(t, \rho, s)=J^{0}(t, s)\left(1+\Delta d(t, s)\left(\varepsilon\left(\rho+h_{\varepsilon}(t, s)\right)\right)\right.
$$

$$
\left.+\sum_{i=2}^{n-1}\left(\varepsilon\left(\rho+h_{\varepsilon}(t, s)\right)\right)^{i} j_{i}(t, s)\right)
$$

with some given functions $j_{i}$ depending on $\gamma_{t}$; see [3], (44), page 537 and also page 538. By the change of variable formula [see [3], (39)], we obtain

$$
\begin{aligned}
\partial_{t} u^{\varepsilon}(t, x)= & \left(V(t, \mathbf{S}(t, x)) \varepsilon^{-1}\right. \\
& \left.-\partial_{t}^{\Gamma} h_{\varepsilon}(t, \mathbf{S}(t, x))\right) \partial_{\rho} \tilde{u}^{\varepsilon}(t, \rho(t, x), \mathbf{S}(t, x)) \\
& +\partial_{t}^{\Gamma} \tilde{u}^{\varepsilon}(t, \rho(t, x), \mathbf{S}(t, x)), \\
\Delta u^{\varepsilon}(t, x)= & \left(\varepsilon^{-2}+\left|\nabla^{\Gamma} h_{\varepsilon}(t, \mathbf{S}(t, x))\right|^{2}\right) \partial_{\rho}^{2} \tilde{u}^{\varepsilon}(t, \rho(t, x), \mathbf{S}(t, x)) \\
& +\left(\Delta d(t, x) \varepsilon^{-1}-\Delta^{\Gamma} h_{\varepsilon}(t, \mathbf{S}(t, x))\right) \partial_{\rho} \tilde{u}^{\varepsilon}(t, \rho(t, x), \mathbf{S}(t, x)) \\
& -2 \nabla^{\Gamma} h_{\varepsilon}(t, \mathbf{S}(t, x)) \cdot \nabla^{\Gamma} \partial_{\rho} \tilde{u}^{\varepsilon}(t, \rho(t, x), \mathbf{S}(t, x)) \\
& +\Delta^{\Gamma} \tilde{u}^{\varepsilon}(t, \rho(t, x), \mathbf{S}(t, x)),
\end{aligned}
$$

where $\tilde{u}^{\varepsilon}=\tilde{u}^{\varepsilon}(t, \rho, s)$ is the function $u^{\varepsilon}=u^{\varepsilon}(t, x)$ viewed under the coordinate $(t, \rho, s)$ defined by (2.19). In the following, we will denote $\tilde{u}^{\varepsilon}$ by $u$ for simplicity. Therefore, from (2.15), we have

$$
\begin{aligned}
0= & {\left[\partial_{\rho}^{2} u+f(u)\right]+\varepsilon\left[(-V(t, s)+\Delta d) \partial_{\rho} u-\lambda_{\varepsilon}(t)\right] } \\
& +\varepsilon^{2}\left[\left(\Delta^{\Gamma} u-\partial_{t}^{\Gamma} u\right)+\left(\partial_{t}^{\Gamma} h_{\varepsilon}-\Delta^{\Gamma} h_{\varepsilon}\right) \partial_{\rho} u\right] \\
& +\varepsilon^{2}\left[\left|\nabla^{\Gamma} h_{\varepsilon}\right|^{2} \partial_{\rho}^{2} u-2 \nabla^{\Gamma} h_{\varepsilon} \cdot \nabla^{\Gamma} \partial_{\rho} u\right]+\varepsilon^{2} v(t) .
\end{aligned}
$$

Suppose that $u$ and $h_{\varepsilon}$ have the inner asymptotic expansions:

$$
\begin{aligned}
& u(t, \rho, s)=m(\rho)+\varepsilon u_{0}(t, \rho, s)+\varepsilon^{2} u_{1}(t, \rho, s)+\varepsilon^{3} u_{2}(t, \rho, s)+\cdots, \\
& \varepsilon h_{\varepsilon}(t, s)=\varepsilon h_{1}(t, s)+\varepsilon^{2} h_{2}(t, s)+\varepsilon^{3} h_{3}(t, s)+\cdots,
\end{aligned}
$$

for $(t, \rho, s) \in\left[0, T^{v}\right] \times \mathbb{R} \times \mathcal{S}$, respectively, where $m$ is the standing wave solution determined by (1.2). On the other hand, assume that $\lambda_{\varepsilon}$ and $u^{ \pm}$have the outer 
asymptotic expansions:

$$
\begin{aligned}
& \lambda_{\varepsilon}(t)=\lambda_{0}(t)+\varepsilon \lambda_{1}(t)+\varepsilon^{2} \lambda_{2}(t)+\varepsilon^{3} \lambda_{3}(t)+\cdots, \\
& u^{ \pm}(t)= \pm 1+\varepsilon u_{0}^{ \pm}(t)+\varepsilon^{2} u_{1}^{ \pm}(t)+\varepsilon^{3} u_{2}^{ \pm}(t)+\cdots,
\end{aligned}
$$

for $t \in\left[0, T^{v}\right]$, respectively. As we mentioned in Section 1.3 after formulating Theorem 1.1, the inner expansion of $u^{\varepsilon}$ is that given near the interface. The transition from $(+1)$-phase to $(-1)$-phase near the interface is sharp. To relax this, we observe the solution $u^{\varepsilon}$ in a stretched spatial variable $\rho$ and make its expansion in this scale. To complete this procedure, we need to determine the boundary values of the inner solution at $\rho= \pm \infty$ (i.e., the limits as $\rho \rightarrow \pm \infty$ ), and the outer solution provides this information. This was already seen for $u_{0}^{ \pm}(t)$ in the heuristic argument in Section 2.1.

Furthermore, let us note that $\Delta d(t, x)$ is expanded into

$$
\begin{aligned}
& \left.\Delta d(t, x)\right|_{x=X_{0}(t, s)+\varepsilon\left(\rho+h_{\varepsilon}(t, s)\right) \mathbf{n}(t, s)} \\
& \quad=\kappa(t, s)-\varepsilon\left(\rho+h_{\varepsilon}(t, s)\right) b(t, s)+\sum_{i \geq 2} \varepsilon^{i} b_{i}(t, s)\left(\rho+h_{\varepsilon}(t, s)\right)^{i},
\end{aligned}
$$

where $b_{i}(t, s), i \geq 2$ are some functions depending only on $\gamma_{t}$; see (40) of [3].

2.4. Inductive scheme to determine coefficients. In this subsection, for a fixed $K>\max (n+2,6)$, we construct functions $\left\{u_{k}\right\}_{k=0}^{K},\left\{h_{k}\right\}_{k=0}^{K},\left\{\lambda_{k}\right\}_{k=0}^{K}$ and $\left\{u_{k}^{ \pm}\right\}_{k=0}^{K}$ appearing in the above expansions (2.24) and (2.25) of $u, h_{\varepsilon}, \lambda_{\varepsilon}$ and $u^{ \pm}$defined for $t \in\left[0, T^{v}\right]$ in such a manner that all $k$ th order terms [i.e., those of order $O\left(\varepsilon^{k}\right)$ ] vanish when we substitute these formal expansions in (2.23), where we set $h_{0}=0$ for convenience. In fact, setting

$$
v_{k}=\left(u_{k}, h_{k}, \lambda_{k}, u_{k}^{ \pm}\right), \quad k=0,1, \ldots, K,
$$

$v_{k}$ will be inductively determined as follows: For $k=0, \lambda_{0}$ will be defined by (2.29) below, $u_{0}$ by (2.32) and $u_{0}^{ \pm}$by (2.34), respectively. This determines $v_{0}$. For $k \geq 1$, once we know $\left\{v_{i}\right\}_{i=0}^{k-1}, h_{k}$ is determined by solving equation (2.44) and $\lambda_{k}$ by (2.43) knowing $h_{k}$ additionally, respectively. Furthermore, $u_{k}$ is defined by (2.38) knowing $h_{k}$ and $\lambda_{k}$, while $u_{k}^{ \pm}$are determined by (2.41).

When we insert the expansion of $u$ in (2.24) into (2.23), the term of order $O(1)$ needs to satisfy $m^{\prime \prime}(\rho)+f(m(\rho))=0$ so that we took the leading term of $u$ as $m(\rho)$.

Let us start the procedure to determine $\nu_{0}$. For the term of order $O(\varepsilon)$ in $(2.23)$ to vanish, recalling (2.26), we have

$$
\mathcal{L} u_{0}=(-V+\kappa) m^{\prime}-\lambda_{0}(t),
$$

where $\mathcal{L}$ is the linearized operator of $-\left(\partial_{\rho}^{2} u+f(u)\right)$ around $m$ defined as

$$
\mathcal{L} u_{0}=-\partial_{\rho}^{2} u_{0}-f^{\prime}(m) u_{0}, \quad \rho \in \mathbb{R} .
$$


Suggested by (2.4), we first define $\lambda_{0}(t)$ as

$$
\lambda_{0}(t)=\frac{1}{\bar{\sigma}\left|\gamma_{t}^{v}\right|} \int_{\gamma_{t}^{v}} \kappa(t, \bar{s}) d \bar{s}-\frac{|D|}{2 \bar{\sigma}\left|\gamma_{t}^{v}\right|} v(t) .
$$

Note that $\int_{\gamma_{t}^{v}} \kappa(t, \bar{s}) d \bar{s}=\int_{\mathcal{S}} \kappa(t, s) J^{0}(t, s) d s$ and $\left|\gamma_{t}^{v}\right|=\int_{\mathcal{S}} J^{0}(t, s) d s$ by the change of variables $\bar{s}=X_{0}(t, s)$. Then, from the solvability condition $\int \mathcal{L} u_{0} \times$ $m^{\prime} d \rho=0$ for (2.28), $V$ needs to satisfy

$$
-V(t, s)+\kappa(t, s)=\lambda_{0}(t) \bar{\sigma}, \quad(t, s) \in\left[0, T^{v}\right] \times \mathcal{S},
$$

where $\bar{\sigma}=2\left(\int_{\mathbb{R}} m^{\prime}(\rho)^{2} d \rho\right)^{-1}$. This combined with (2.29) leads to the evolutional law (2.6) of $\gamma_{t}^{v}$.

Next, to determine $u_{0}$, we note the following fact: Since $m_{1}(\rho):=m^{\prime}(\rho)$ and $m_{2}(\rho):=m^{\prime}(\rho) \int_{0}^{\rho} \frac{1}{m^{\prime}(y)^{2}} d y$ are linearly independent two solutions of SturmLiouville second-order differential equation $\mathcal{L} u(\rho)=0$ with Wronskian $m_{1}^{\prime} m_{2}-$ $m_{1} m_{2}^{\prime}=-1$, the solution of the equation $\mathcal{L} u(\rho)=g(\rho)$ satisfying $u(0)=0$ is unique and given by

$$
\begin{aligned}
u(\rho)= & \left(-\int_{0}^{\rho} m^{\prime}(y) g(y) d y\right) m^{\prime}(\rho) \int_{0}^{\rho} \frac{d y}{m^{\prime}(y)^{2}} \\
& +\left(\int_{0}^{\rho} m^{\prime}(y)\left(\int_{0}^{y} \frac{d z}{m^{\prime}(z)^{2}}\right) g(y) d y\right) m^{\prime}(\rho) .
\end{aligned}
$$

Let us determine $u_{0}(t, \rho, s)$ and $u_{0}^{ \pm}(t)$. The function $u_{0}(t, \rho, s)$ satisfies (2.28). To solve this equation, let $\theta_{1}$ be a unique solution of $\mathcal{L} \theta_{1}=1-m^{\prime} \bar{\sigma}$, namely, from (2.31) with $g=1-m^{\prime} \bar{\sigma}$,

$$
\begin{aligned}
\theta_{1}(\rho)= & \left(-\int_{0}^{\rho} m^{\prime}(y)\left(1-m^{\prime}(y) \bar{\sigma}\right) d y\right) m^{\prime}(\rho) \int_{0}^{\rho} \frac{1}{m^{\prime}(y)^{2}} d y \\
& +\left(\int_{0}^{\rho} m^{\prime}(y)\left(\int_{0}^{y} \frac{1}{m^{\prime}(z)^{2}} d z\right)\left(1-m^{\prime}(y) \bar{\sigma}\right) d y\right) m^{\prime}(\rho) .
\end{aligned}
$$

Then, noting (2.30), $u_{0}(t, \rho, s)$ is given by

$$
u_{0}(t, \rho, s)=-\lambda_{0}(t) \theta_{1}(\rho) .
$$

Furthermore, since $\lim _{\rho \rightarrow \pm \infty}\left|g(t, \rho, s)+\lambda_{0}(t)\right|=O\left(e^{-\zeta|\rho|}\right), \zeta>0$ holds for the right-hand side $g(t, \rho, s)$ of (2.28), by Lemma 3 in [3], it follows that

$$
\lim _{\rho \rightarrow \pm \infty} \partial_{\rho}^{m} \partial_{s}^{\mathbf{n}_{2}} \partial_{t}^{l}\left(u_{0}(t, \rho, s)-\frac{\lambda_{0}(t)}{f^{\prime}( \pm 1)}\right)=O\left(e^{-\zeta|\rho|}\right),
$$

for all $(m, \mathbf{n}, l) \in \mathbb{Z}_{+} \times \mathbb{Z}_{+}^{n-1} \times \mathbb{Z}_{+}$. Therefore, we define

$$
u_{0}^{ \pm}(t):=\frac{\lambda_{0}(t)}{f^{\prime}( \pm 1)}
$$


Now, let us determine $v_{k}$ for $k \geq 1$ assuming that $\left\{v_{i}\right\}_{i=0}^{k-1}$ are known. For the term of order $O\left(\varepsilon^{k+1}\right)$ in (2.23) to vanish, it is necessary to have

$$
\begin{aligned}
\mathcal{L} u_{k}= & A^{k-1}+\left(\partial_{t}^{\Gamma}-\Delta^{\Gamma}-b(t, s)\right) h_{k} m^{\prime}-b(t, s) \rho m^{\prime} 1_{\{k=1\}} \\
& +\left(2 \cdot 1_{\{k \geq 2\}}+1_{\{k=1\}}\right) \nabla^{\Gamma} h_{1} \cdot \nabla^{\Gamma} h_{k} m^{\prime \prime}-\lambda_{k}(t)+v(t) 1_{\{k=1\}},
\end{aligned}
$$

where $b(t, s)$ is defined by (2.14), $A^{k-1}=A^{k-1}\left(\lambda_{0}, u_{i}, h_{i}, 0 \leq i \leq k-1\right)$ is given by

$$
\begin{aligned}
A^{k-1}= & \lambda_{0}(t) \bar{\sigma} \partial_{\rho} u_{k-1} \\
& +\sum_{l=2}^{k} b_{l}(t, s) \sum_{\substack{i_{1}, \ldots, i_{l} \geq 1, j \geq-1, i_{1}+\cdots+i_{l}+j=k-1}} \tilde{h}_{i_{1}} \cdots \tilde{h}_{i_{l}} \partial_{\rho} u_{j} \\
& -\partial_{t}^{\Gamma} u_{k-2}+\Delta^{\Gamma} u_{k-2} \\
& +\sum_{i=1}^{k-1}\left(\partial_{t}^{\Gamma} h_{i}-\Delta^{\Gamma} h_{i}-b(t, s) \tilde{h}_{i}\right) \partial_{\rho} u_{k-1-i} \\
& +\sum_{\substack{i, j \geq 1, i+j \leq k+1,(i, j) \neq(k, 1),(1, k)}}^{\nabla^{\Gamma} h_{i} \cdot \nabla^{\Gamma} h_{j} \partial_{\rho}^{2} u_{k-(i+j)}} \\
& -2 \sum_{i=1}^{k-1} \nabla^{\Gamma} h_{i} \cdot \nabla^{\Gamma} \partial_{\rho} u_{k-1-i} \\
& +\sum_{l=2}^{k+1} \frac{1}{l !} f^{(l)}(m) \sum_{\substack{i_{1}, \ldots, i_{l} \geq 0, i_{1}+\cdots+i_{l}=k+1-l}} u_{i_{1}} \cdots u_{i_{l}},
\end{aligned}
$$

$\tilde{h}_{i}(t, s):=h_{i}(t, s)+\rho 1_{\{i=1\}}, 1 \leq i \leq K, u_{-1}=m$ and $b_{l}(t, s)$ are defined by (2.26). In particular, $A^{0}\left(\lambda_{0}, u_{0}\right)=\lambda_{0}(t) \bar{\sigma} \partial_{\rho} u_{0}+\frac{1}{2} f^{\prime \prime}(m) u_{0}^{2}$, since $\partial_{t} m=$ $\Delta^{\Gamma} m=0$. We have used (2.26) and (2.30) to have the expansion of the term $-V+\Delta d$.

From the solvability condition $\int \mathcal{L} u_{k} m^{\prime} d \rho=0$ for (2.35) and noting that $\int_{\mathbb{R}} m^{\prime \prime} m^{\prime} d \rho=0$, it follows that $\lambda_{k}$ and $h_{k}$ should satisfy

$$
\begin{aligned}
\lambda_{k}(t)= & \frac{1}{\bar{\sigma}}\left(\partial_{t}^{\Gamma}-\Delta^{\Gamma}-b(t, s)\right) h_{k}+\frac{1}{2} \int_{\mathbb{R}} A^{k-1} m^{\prime}(\rho) d \rho \\
& +\left\{v(t)-\frac{1}{2} b(t, s) \int_{\mathbb{R}} \rho\left(m^{\prime}(\rho)\right)^{2} d \rho\right\} 1_{\{k=1\}} .
\end{aligned}
$$

The next task is to determine $u_{k}(t, \rho, s)$ and $u_{k}^{ \pm}(t)$. Recall that $k \geq 1$ is fixed. From (2.31), it follows that the linear equation $\mathcal{L} u_{k}=\widetilde{A}^{k}$ satisfying $u(t, 0, s)=0$ 
has a unique solution given by

$$
\begin{aligned}
u_{k}(t, \rho, s)= & \left(-\int_{0}^{\rho} m^{\prime}(y) \widetilde{A}^{k}(t, y, s) d y\right) m^{\prime}(\rho) \int_{0}^{\rho} \frac{d y}{m^{\prime}(y)^{2}} \\
& +\left(\int_{0}^{\rho} m^{\prime}(y)\left(\int_{0}^{y} \frac{d z}{m^{\prime}(z)^{2}}\right) \widetilde{A}^{k}(t, y, s) d y\right) m^{\prime}(\rho),
\end{aligned}
$$

where $\widetilde{A}^{k}(t, \rho, s)$ denotes the right-hand side of (2.35). Especially, supposing that $u_{0}^{ \pm}, u_{1}^{ \pm}, \ldots, u_{k-1}^{ \pm}$are determined inductively and $\left\{u_{i}\right\}_{i=0}^{k-1}$ satisfy the following (2.39) with $i$ instead of $k$, from Lemma 3 in [3] and noting that $h_{k}$ are independent of $\rho$ and $m^{\prime}, \rho m^{\prime}, m^{\prime \prime}, \partial_{\rho} u_{i}, \partial_{\rho}^{2} u_{i}, \Delta^{\Gamma} u_{k-2}$ tend to 0 as $|\rho| \rightarrow \infty$, it is easy to check

$$
\partial_{\rho}^{m} \partial_{s}^{\mathbf{n}} \partial_{t}^{l}\left(u_{k}(t, \rho, s)-\frac{g_{k}^{ \pm}(t)}{f^{\prime}( \pm 1)}\right)=O\left(e^{-\zeta|\rho|}\right) \quad \text { as } \rho \rightarrow \pm \infty,
$$

for $(t, s)$, where

$$
\begin{aligned}
g_{k}^{ \pm}(t)= & -\left(\left.A^{k-1}\right|_{\rho= \pm \infty}-\lambda_{k}(t)+v(t) 1_{\{k=1\}}\right) \\
= & -\sum_{j=2}^{k+1} \frac{1}{j !} \sum_{\substack{0 \leq i_{1} \leq i_{2} \leq \cdots \leq i_{j}, i_{1}+i_{2}+\cdots+i_{j}=k-(j-1)}} f^{(j)}( \pm 1) \frac{u_{i_{1}}^{ \pm}(t) \cdots u_{i_{j}}^{ \pm}(t)}{f^{\prime}( \pm 1)^{j}} \\
& +\partial_{t}^{\Gamma} u_{k-2}^{ \pm}(t)+\lambda_{k}(t)-v(t) 1_{\{k=1\}} .
\end{aligned}
$$

This suggests to define

$$
u_{k}^{ \pm}(t):=\frac{g_{k}^{ \pm}(t)}{f^{\prime}( \pm 1)}
$$

We will determine $\lambda_{k}(t)$ in such a way that the term of order $O\left(\varepsilon^{k}\right)$ of the integral

$$
\int_{D}\left(\partial_{t} u_{k}^{\varepsilon}(t, x)-v(t)\right) d x
$$

vanishes, where $u_{k}^{\varepsilon}$ is an approximate solution defined as (3.2) later. In fact, this results in

$$
\begin{aligned}
\lambda_{k}(t)= & \frac{1}{\bar{\sigma}\left|\gamma_{t}^{v}\right|} \int_{\mathcal{S}}\left(\left(\Delta d\left(t, X_{0}(t, s)\right)-\bar{\sigma} \lambda_{0}(t)\right) \Delta d\left(t, X_{0}(t, s)\right) h_{k}(t, s)\right. \\
& \left.-b(t, s) h_{k}(t, s)+\frac{\bar{\sigma}}{2} \int_{\mathbb{R}} A^{k-1} m^{\prime}(\rho) d \rho\right) J^{0}(t, s) d s \\
& +\left(v(t)-\frac{1}{2\left|\gamma_{t}^{v}\right|} \int_{\mathbb{R}} \rho\left(m^{\prime}(\rho)\right)^{2} d \rho \int_{\mathcal{S}} b(t, s) J^{0}(t, s) d s\right) 1_{\{k=1\}} \\
& +\frac{1}{2 \bar{\sigma}\left|\gamma_{t}^{v}\right|} B_{k-1}
\end{aligned}
$$


where $B_{k-1}=B_{k-1}\left(u_{i}, u_{i}^{ \pm}, h_{i}, 0 \leq i \leq k-1\right)$ is a term which is determined from $u_{i}, u_{i}^{ \pm}$and $h_{i}, 0 \leq i \leq k-1$; see Section 3.2, in particular, (3.11) for $B_{k-1}$ and (3.14) below. From (2.37) and (2.43), we define $h_{k}$ as the solution of the following equation:

$$
\begin{aligned}
\left(\partial_{t}^{\Gamma}-\Delta^{\Gamma}-b(t, s)\right) h_{k}(t, s) & \\
- & \frac{1}{\left|\gamma_{t}^{v}\right|} \int_{\mathcal{S}}\left(\left(\Delta d\left(t, X_{0}\left(t, s^{\prime}\right)\right)-\bar{\sigma} \lambda_{0}(t)\right) \Delta d\left(t, X_{0}\left(t, s^{\prime}\right)\right)-b\left(t, s^{\prime}\right)\right) \\
\times & h_{k}\left(t, s^{\prime}\right) J^{0}\left(t, s^{\prime}\right) d s^{\prime} \\
= & -\frac{\bar{\sigma}}{2} \int_{\mathbb{R}} A^{k-1} m^{\prime}(\rho) d \rho+\frac{\bar{\sigma}}{2\left|\gamma_{t}^{v}\right|} \iint_{\mathcal{S} \times \mathbb{R}} A^{k-1} m^{\prime}(\rho) J^{0}\left(t, s^{\prime}\right) d s^{\prime} d \rho \\
& +\left(\frac{\bar{\sigma}}{2}\left(b(t, s)-\frac{1}{\left|\gamma_{t}^{v}\right|} \int_{\mathcal{S}} b\left(t, s^{\prime}\right) J^{0}\left(t, s^{\prime}\right) d s^{\prime}\right) \int_{\mathbb{R}} \rho\left(m^{\prime}(\rho)\right)^{2} d \rho\right) 1_{\{k=1\}} \\
& +\frac{1}{2\left|\gamma_{t}^{v}\right|} B_{k-1} .
\end{aligned}
$$

This is a linear equation for $h_{k}, k \geq 1$; note that the right-hand side is determined from $\lambda_{0}, u_{i}, u_{i}^{ \pm}$and $h_{i}$ with $0 \leq i \leq k-1$. When $k=1$, nonlinear term $\left|\nabla^{\Gamma} h_{1}\right|^{2}$ appears in (2.35), however, this disappears by the relation $\int_{\mathbb{R}} m^{\prime}(\rho) m^{\prime \prime}(\rho) d \rho=0$. We will study equation (2.44) in Section 3.3.3.

2.5. Heuristic argument for the equation with spatially dependent noise. To digress a little, we discuss the equation with a spatially dependent noise at a heuristic level. Let an $x$-dependent noise $\dot{w}^{\varepsilon}(t, x)$, which is also denoted by $v(t, x)$ as above, is given, and assume that it is smooth in $x$ and satisfies the vanishing condition $\int_{D} v(t, x) d x=0$. With such noise, consider the equation

$$
\frac{\partial u^{\varepsilon}}{\partial t}=\Delta u^{\varepsilon}+\varepsilon^{-2}\left(f\left(u^{\varepsilon}\right)-f_{D} f\left(u^{\varepsilon}\right)\right)+\varepsilon^{-1} v(t, x) \quad \text { in } D \times \mathbb{R}_{+},
$$

instead of (1.1) or (2.5). This equation has the precise mass conservation law (1.5) differently from (1.6) for equation (1.1). The scaling of the noise should be as in (2.45) and this is different from that for (1.1), but similar to the stochastic AllenCahn equation as we mentioned at the end of Section 1.1 and at the beginning of Section 2.1. We expect that the sharp interface limit holds for (2.45) and the evolution of the limit hypersurface $\gamma_{t}$ is governed by the equation

$$
V=\kappa-f_{\gamma_{t}} \kappa+\bar{\sigma}\left(\dot{w}(t, x)-f_{\gamma_{t}} \dot{w}(t)\right), \quad x \in \gamma_{t} .
$$

The noise in the limit dynamics is simply additive, but the volume inside $\gamma_{t}$ is preserved. 
The heuristic argument to derive (2.46) from (2.45) goes as follows. Our guess for the behavior of the solution $u^{\varepsilon}$ of (2.45) is modified from (2.2) to the following:

$$
u^{\varepsilon}(t, x)=m\left(\frac{d(t, x)}{\varepsilon} ; a^{\varepsilon}(t)-\varepsilon v(t, x)\right)+O(\varepsilon) .
$$

Note that $\varepsilon^{2} v(t)$ is now replaced by $\varepsilon v(t, x)$. Then, in the computation below (2.2), the first line is the same and the lines below are modified as follows:

$$
\begin{aligned}
0 \approx & \frac{1}{\varepsilon^{2}} \int_{D}\left\{m^{\prime \prime}\left(\frac{d}{\varepsilon}\right)+\varepsilon m^{\prime}\left(\frac{d}{\varepsilon}\right) \Delta d+f\left(m\left(\frac{d}{\varepsilon}\right)\right)-a^{\varepsilon}(t)+\varepsilon v(t, x)\right\} d x \\
& -2 \int_{D} \frac{\partial m^{\prime}}{\partial a}\left(\frac{d}{\varepsilon}\right) \nabla d \cdot \nabla v d x+O(\varepsilon) \\
\approx & \frac{1}{\varepsilon^{2}} \int_{D} m^{\prime}\left(\frac{d}{\varepsilon}\right)\left\{-c\left(a^{\varepsilon}(t)-\varepsilon v(t, x)\right)+\varepsilon \Delta d\right\} d x \\
\approx & \left(m_{+}^{*}-m_{-}^{*}\right) \int_{\gamma_{t}}\left\{-c_{0} \frac{a^{\varepsilon}(t)}{\varepsilon}+\kappa(t, \bar{s})\right\} d \bar{s}+\left(m_{+}^{*}-m_{-}^{*}\right) \int_{\gamma_{t}} c_{0} v(t, \bar{s}) d \bar{s} .
\end{aligned}
$$

In the first line, we have used the vanishing condition $\int_{D} v(t, x) d x=0$ and need to compute the derivatives of $m$ in $a$, since it contains $v(t, x)$. However, the integral containing $\frac{\partial m^{\prime}}{\partial a}$ is expected to behave as $O(\varepsilon)$. Therefore, instead of (2.4), we finally obtain

$$
2 c_{0} \lambda_{0}(t)\left|\gamma_{t}\right|=2 \int_{\gamma_{t}} \kappa(t) d \bar{s}+2 c_{0} \int_{\gamma_{t}} v(t, \bar{s}) d \bar{s} .
$$

On the other hand, instead of (2.30), we would have

$$
V=\kappa-\bar{\sigma}\left(\lambda_{0}(t)-v(t, \bar{s})\right) \quad \text { on } \gamma_{t} .
$$

Indeed, to show this, we compute similar to Section 2.4. In particular, noting that $\varepsilon^{2} v(t)$ in (2.23) is replaced by $\varepsilon v(t, x),(2.28)$ is changed as

$$
\mathcal{L} u_{0}=(-V+\kappa) m^{\prime}-\lambda_{0}(t)+v(t, s), \quad s \in \mathcal{S} .
$$

Then, since $\int \mathcal{L} u_{0} m^{\prime} d \rho=0, \bar{\sigma} \equiv c_{0}=2\left(\int_{\mathbb{R}} m^{\prime}(\rho)^{2} d \rho\right)^{-1}$ and $\int_{\mathbb{R}} m^{\prime} d \rho=2$, we obtain (2.49). Recalling $c_{0}=\bar{\sigma}$, we obtain (2.46) with $v$ instead of $\dot{w}$ from (2.48) and (2.49).

We will not discuss the case with the spatially dependent noise in the present paper, since the mean curvature motion with a spatial dependent noise is still not well studied.

\section{Approximate solutions and their estimates.}

3.1. Approximate solutions. Once all $\left\{v_{i}\right\}_{i=0}^{K}$ are determined, for $k=0,1$, $\ldots, K$, we can define approximations $u_{k, \varepsilon}^{\text {in }}, u_{k, \varepsilon, \pm}^{\text {out }}, h_{k}^{\varepsilon}, \lambda_{k}^{\varepsilon}$ and $\rho_{k, \varepsilon}$ of $u$ both in 
the sense of inner and outer solutions, $h_{\varepsilon}, \lambda_{\varepsilon}$ and $\rho_{\varepsilon}$, respectively, by cutting the expansions (2.24) and (2.25) after the $k+1$ th or $k$ th terms:

$$
\begin{aligned}
u_{k, \varepsilon}^{\text {in }}(t, x) & :=m(\rho)+\sum_{i=0}^{k} \varepsilon^{i+1} u_{i}(t, \rho, \mathbf{S}(t, x)), \\
u_{k, \varepsilon, \pm}^{\text {out }}(t) & := \pm 1+\sum_{i=0}^{k} \varepsilon^{i+1} u_{i}^{ \pm}(t), \\
\varepsilon h_{k}^{\varepsilon}(t, s) & =\sum_{i=1}^{k} \varepsilon^{i} h_{i}(t, s), \\
\lambda_{k}^{\varepsilon}(t) & :=\sum_{i=0}^{k} \varepsilon^{i} \lambda_{i}(t), \\
\rho & =\rho_{k, \varepsilon}(t, x):=\frac{1}{\varepsilon}\left(d(t, x)-\varepsilon h_{k}^{\varepsilon}(t, \mathbf{S}(t, x))\right) .
\end{aligned}
$$

Let $\eta \in C^{\infty}(\mathbb{R})$ be a function satisfying the conditions: $\eta(s)=1$ for $|s| \leq \delta$, $\eta(s)=0$ for $|s| \geq 2 \delta$ and $0 \leq s \eta^{\prime}(s) \leq 4$ for $\delta \leq|s| \leq 2 \delta$. Then define the approximate solutions $u_{k}^{\varepsilon}(t, x)$ as follows by connecting the inner and outer approximate solutions:

$$
\begin{aligned}
u_{k}^{\varepsilon}(t, x):= & \eta(d(t, x)) u_{k, \varepsilon}^{i n}(t, x) \\
& +(1-\eta(d(t, x)))\left(u_{k, \varepsilon,+}^{\text {out }}(t) 1_{\{d>0\}}+u_{k, \varepsilon,-}^{\text {out }}(t) 1_{\{d<0\}}\right) .
\end{aligned}
$$

3.2. Derivation of (2.43). Now we consider the integral (2.42), which is the left-hand side of (1.7) times $|D|$ with $u^{\varepsilon}$ and $\alpha \dot{w}^{\varepsilon}$ replaced by $u_{k}^{\varepsilon}$ and $v$, respectively, and expand it in $\varepsilon$ to obtain (2.43). Let us decompose the time derivative of the mass of $u_{k}^{\varepsilon}$ over $D$ into

$$
\begin{aligned}
\int_{D} \partial_{t} u_{k}^{\varepsilon}(t, x) d x= & \int_{|d(t, x)| \geq 3 \delta} \partial_{t} u_{k}^{\varepsilon}(t, x) d x \\
& +\int_{|\rho| \geq \frac{\delta}{\varepsilon}} \partial_{t} u_{k}^{\varepsilon}(t, x) d x+\int_{|\rho|<\frac{\delta}{\varepsilon}} \partial_{t} u_{k}^{\varepsilon}(t, x) d x \\
= & I+I I+I I I,
\end{aligned}
$$

where $\delta>0$ is chosen in Section 2.2, $\left\{|\rho| \geq \frac{\delta}{\varepsilon}\right\}=\left\{x \in V_{3 \delta}^{t}:|\rho(t, x)| \geq \frac{\delta}{\varepsilon}\right\},\{|\rho|<$ $\left.\frac{\delta}{\varepsilon}\right\}=\left\{x \in V_{3 \delta}^{t}:|\rho(t, x)|<\frac{\delta}{\varepsilon}\right\}$ and $V_{3 \delta}^{t}:=\{x \in D:|d(t, x)|<3 \delta\}$. From now on, we choose a sufficiently small $\varepsilon_{0}^{*}=\varepsilon_{0}^{*}(\delta, K, v)>0$ such that

$$
\sup _{s \in \mathcal{S}, t \in[0, T]}\left|\varepsilon h_{k}^{\varepsilon}(t, s)\right| \leq \frac{\delta}{2}
$$


holds for all $0<\varepsilon \leq \varepsilon_{0}^{*}$. This is possible for each $v$. Thus $|d(t, x)| \geq \frac{\delta}{2}$ follows from $|\rho(t, x)| \geq \frac{\delta}{\varepsilon}$ and for any point $(t, x)$ where either $|d(t, x)| \geq 3 \delta$ or $|\rho(t, x)| \geq$ $\frac{\delta}{\varepsilon},|d(t, x)| \geq \frac{\delta}{2}$ holds. Noting that each inner solution $u_{i}$ converges to its associated outer solution $u_{i}^{ \pm}$with an error $O\left(e^{-\zeta|\rho|}\right)$ as $|\rho| \rightarrow \infty$, and setting

$$
\begin{aligned}
& D_{\varepsilon}^{+}(t)=\{x \in D: d(t, x) \geq 3 \delta\} \cup\{x \in D:|d(t, x)|<3 \delta, \rho(t, x)>0\}, \\
& D_{\varepsilon}^{-}(t)=D \backslash \bar{D}_{\varepsilon}^{+}(t),
\end{aligned}
$$

we have

$$
\begin{aligned}
I+I I= & \left(u_{k, \varepsilon,+}^{\text {out }}\right)^{\prime}(t)\left|D_{\varepsilon}^{+}(t)\right|+\left(u_{k, \varepsilon,-}^{\text {out }}\right)^{\prime}(t)\left|D_{\varepsilon}^{-}(t)\right| \\
& -\left(u_{k, \varepsilon,+}^{\text {out }}\right)^{\prime}(t) \int_{|\rho|<\frac{\delta}{\varepsilon}} 1_{\{\rho>0\}} d x \\
& -\left(u_{k, \varepsilon,-}^{\text {out }}\right)^{\prime}(t) \int_{|\rho|<\frac{\delta}{\varepsilon}} 1_{\{\rho<0\}} d x+O\left(e^{-\zeta \delta / \varepsilon}\right),
\end{aligned}
$$

as $\varepsilon \downarrow 0$, where ' means the derivative in $t$ and we have used (2.39) which gives the error term $O\left(e^{-\zeta \delta / \varepsilon}\right)$. Concerning the term III, change of variables and (81) of [3] lead us to

$$
\begin{aligned}
I I I= & \int_{\mathcal{S}} \int_{|\rho|<\frac{\delta}{\varepsilon}}\left(\left(V(t, s) \varepsilon^{-1}-\partial_{t}^{\Gamma} h_{k}^{\varepsilon}\right) \partial_{\rho} u_{k}^{\varepsilon}\right) \varepsilon J^{\varepsilon}(t, \rho, s) d \rho d s \\
& +\int_{\mathcal{S}} \int_{|\rho|<\frac{\delta}{\varepsilon}} \partial_{t}^{\Gamma} u_{k}^{\varepsilon} \varepsilon J^{\varepsilon}(t, \rho, s) d \rho d s .
\end{aligned}
$$

As a result, we obtain

$$
\int_{D} \partial_{t} u_{k}^{\varepsilon}(t, x) d x=(A)+(B)+(C)+O\left(e^{-\zeta \delta / \varepsilon}\right),
$$

where

$$
\begin{aligned}
(A)= & \left(u_{k, \varepsilon,+}^{\text {out }}\right)^{\prime}(t)\left|D_{\varepsilon}^{+}(t)\right|+\left(u_{k, \varepsilon,-}^{\text {out }}\right)^{\prime}(t)\left|D_{\varepsilon}^{-}(t)\right|, \\
(B)= & -\int_{|\rho|<\frac{\delta}{\varepsilon}}\left(\left(u_{k, \varepsilon,+}^{\text {out }}\right)^{\prime}(t) 1_{\{\rho>0\}}+\left(u_{k, \varepsilon,-}^{\text {out }}\right)^{\prime}(t) 1_{\{\rho<0\}}\right) d x \\
& +\int_{\mathcal{S}} \int_{|\rho|<\frac{\delta}{\varepsilon}} \partial_{t}^{\Gamma} u_{k}^{\varepsilon}(t, \rho, s) \varepsilon J^{\varepsilon}(t, \rho, s) d \rho d s, \\
(C)= & \int_{\mathcal{S}} \int_{|\rho|<\frac{\delta}{\varepsilon}}\left(V(t, s) \varepsilon^{-1}-\partial_{t}^{\Gamma} h_{k}^{\varepsilon}(t, s)\right) \partial_{\rho} u_{k}^{\varepsilon}(t, \rho, s) \varepsilon J^{\varepsilon}(t, \rho, s) d \rho d s .
\end{aligned}
$$

In the following, we rewrite these three terms $(A),(B)$ and $(C)$. First, $(A)$ can be rewritten as follows: Let us denote by $J(t, r, s)$ the Jacobian of the map (2.10). By equation (44) and page 538 of [3], we have

$$
J(t, r, s)=J^{0}(t, s)\left(1+\Delta d\left(t, X_{0}(t, s)\right) r+\sum_{i=2}^{n-1} r^{i} j_{i}(t, s)\right),
$$


for some functions $j_{i}(t, s)$; see also (2.20). By (3.5), we get

$$
\begin{aligned}
\left|D_{\varepsilon}^{-}(t)\right| & =\left|D_{t}\right|+\int_{\mathcal{S}} \int_{0}^{\varepsilon h_{k}^{\varepsilon}(t, s)} J(t, r, s) d r d s \\
& =\left|D_{t}\right|+\int_{\mathcal{S}} \varepsilon h_{k}^{\varepsilon}(t, s) J^{0}(t, s) d s+\left(A_{1}\right), \\
\left|D_{\varepsilon}^{+}(t)\right| & =|D|-\left|D_{\varepsilon}^{-}(t)\right|=|D|-\left|D_{t}\right|-\int_{\mathcal{S}} \varepsilon h_{k}^{\varepsilon}(t, s) J^{0}(t, s) d s-\left(A_{1}\right),
\end{aligned}
$$

where $D_{t}$ denotes the inside surrounded by the hypersurface $\gamma_{t}^{v}$ and

$$
\begin{aligned}
\left(A_{1}\right)= & \frac{1}{2} \int_{\mathcal{S}} \Delta d\left(t, X_{0}(t, s)\right)\left(\varepsilon h_{k}^{\varepsilon}(t, s)\right)^{2} J^{0}(t, s) d s \\
& +\sum_{i=2}^{n-1} \frac{1}{i+1} \int_{\mathcal{S}} j_{i}(t, s)\left(\varepsilon h_{k}^{\varepsilon}(t, s)\right)^{i+1} J^{0}(t, s) d s .
\end{aligned}
$$

Recalling the expansions of $u_{k, \varepsilon, \pm}^{\text {out }}$ and $\varepsilon h_{k}^{\varepsilon}$ given in (3.1), we can decompose $(A)$ into the sum of the following two parts, namely,

$$
(A)=\sum_{i=1}^{k} \varepsilon^{i} B_{i-1}^{A}+\sum_{i=k+1}^{\infty} \varepsilon^{i} \bar{B}_{k, i}^{A},
$$

where $B_{i-1}^{A}$ is the term of order $O\left(\varepsilon^{i}\right)$ determined from $d, u_{j}^{ \pm}$and $h_{j}, 0 \leq j \leq i-1$ and $\bar{B}_{k, i}^{A}$ denotes the term of order $O\left(\varepsilon^{i}\right)$ determined from $d, u_{j}^{ \pm}, h_{j}, 0 \leq j \leq k$ for $i \geq k+1$.

For $(B)$, using (2.20) and the expansions of $u_{k}^{\varepsilon}=u_{k, \varepsilon}^{i n}$ for $|\rho|<\frac{\delta}{\varepsilon}$ and $u_{k, \varepsilon, \pm}^{\text {out }}$ given in (3.1), we obtain

$$
\begin{aligned}
(B)= & \int_{\mathcal{S}} \int_{|\rho|<\frac{\delta}{\varepsilon}}\left(\partial_{t}^{\Gamma} u_{k}^{\varepsilon}-\left(u_{k, \varepsilon,+}^{\text {out }}\right)^{\prime}(t) 1_{\{\rho>0\}}-\left(u_{k, \varepsilon,-}^{\text {out }}\right)^{\prime}(t) 1_{\{\rho<0\}}\right) \\
& \times \varepsilon J^{\varepsilon}(t, \rho, s) d \rho d s \\
= & \sum_{i=0}^{k} \int_{\mathcal{S}} \int_{|\rho|<\frac{\delta}{\varepsilon}} \varepsilon^{i+2}\left(\partial_{t}^{\Gamma} u_{i}(t, \rho, s)-\left(u_{i}^{+}\right)^{\prime}(t) 1_{\{\rho>0\}}-\left(u_{i}^{-}\right)^{\prime}(t) 1_{\{\rho<0\}}\right) \\
& \times\left(1+\varepsilon\left(\rho+\varepsilon h_{k}^{\varepsilon}(t, s)\right) \Delta d+\sum_{l=2}^{n-1} \varepsilon^{l}\left(\rho+\varepsilon h_{k}^{\varepsilon}(t, s)\right)^{l} j_{l}(t, s)\right) \\
& \times J^{0}(t, s) d \rho d s \\
= & \left(B_{1}\right)-\left(B_{2}\right),
\end{aligned}
$$

where $\left(B_{1}\right)$ and $\left(B_{2}\right)$ are defined as the middle of (3.7) with the integral region $\left\{|\rho|<\frac{\delta}{\varepsilon}\right\}$ replaced by $\mathbb{R}$ and $\left\{|\rho| \geq \frac{\delta}{\varepsilon}\right\}$, respectively. Note that both $\left(B_{1}\right)$ and $\left(B_{2}\right)$ 
are finite, since we have from compatibility condition (2.39) and (2.41):

$$
\left(\partial_{t}^{\Gamma} u_{i}(t, \rho, s)-\left(u_{i}^{+}\right)^{\prime}(t) 1_{\{\rho>0\}}-\left(u_{i}^{-}\right)^{\prime}(t) 1_{\{\rho<0\}}\right)=O\left(e^{-\zeta|\rho|}\right),
$$

as $\rho \rightarrow \pm \infty$ for $(t, s)$. Then, similar to $(A)$, we can decompose $\left(B_{1}\right)$ into the sum of two parts and we obtain

$$
(B)=\sum_{i=2}^{k} \varepsilon^{i} B_{i-2}^{B}(t)+\sum_{i=k+1}^{\infty} \varepsilon^{i} \bar{B}_{k, i}^{B}(t)-\left(B_{2}\right),
$$

where $B_{i-2}^{B}$ is the term of order $O\left(\varepsilon^{i}\right)$ determined from $d, u_{j}, u_{j}^{ \pm}$and $h_{j}, 0 \leq j \leq$ $i-2$ and $\bar{B}_{k, i}^{B}$ denotes the term of order $O\left(\varepsilon^{i}\right)$ determined from $d, u_{j}, u_{j}^{ \pm}, h_{j}$, $0 \leq j \leq k$ for $i \geq k+1$.

For $(C)$, note that $V(t, s)=\Delta d\left(t, X_{0}(t, s)\right)-\bar{\sigma} \lambda_{0}(t)$ from (2.13) and (2.30), and $\partial_{\rho} u_{k}^{\varepsilon}=\partial_{\rho} u_{k, \varepsilon}^{i n}=\partial_{\rho} m+\sum_{i=0}^{k} \varepsilon^{i+1} \partial_{\rho} u_{i}$ from (3.1). Recalling (2.20) again, we have

$$
\begin{aligned}
(C)= & \int_{\mathcal{S}} \int_{|\rho|<\frac{\delta}{\varepsilon}}\left\{\left(\Delta d\left(t, X_{0}(t, s)\right)-\bar{\sigma} \lambda_{0}(t)\right)-\partial_{t}^{\Gamma} \sum_{i=1}^{k} \varepsilon^{i} h_{i}(t, s)\right\} \\
& \times\left\{m^{\prime}(\rho)+\sum_{i=0}^{k} \varepsilon^{i+1} \partial_{\rho} u_{i}\right\} \\
& \times\left\{1+\Delta d(t, s)\left(\varepsilon\left(\rho+h_{k}^{\varepsilon}(t, s)\right)\right)+\sum_{i=2}^{n-1}\left(\varepsilon\left(\rho+h_{k}^{\varepsilon}(t, s)\right)\right)^{i} j_{i}(t, s)\right\} \\
& \times J^{0}(t, s) d \rho d s \\
= & \left(C_{1}\right)-\left(C_{2}\right),
\end{aligned}
$$

where $\left(C_{1}\right)$ and $\left(C_{2}\right)$ are defined by the above line with the integral region $\{|\rho|<$ $\left.\frac{\delta}{\varepsilon}\right\}$ replaced by $\mathbb{R}$ and $\left\{|\rho| \geq \frac{\delta}{\varepsilon}\right\}$, respectively. Then, in the term of order $O\left(\varepsilon^{i}\right)$ in the expansion of $\left(C_{1}\right)$ in $\varepsilon$, separating terms containing $h_{i}$ from those containing lower order functions $\left\{v_{j}\right\}_{j \leq i-1}$, and noting $\int_{\mathbb{R}} \partial_{\rho} m d \rho=2$, we can decompose $\left(C_{1}\right)$ into the sum of the following four parts:

$$
\begin{aligned}
\left(C_{1}\right)= & 2 \int_{\mathcal{S}}\left(\Delta d\left(t, X_{0}(t, s)\right)-\bar{\sigma} \lambda_{0}(t)\right) J^{0}(t, s) d s \\
& +2 \sum_{i=1}^{k} \varepsilon^{i} \int_{\mathcal{S}}\left(\left(\Delta d\left(t, X_{0}(t, s)\right)-\bar{\sigma} \lambda_{0}(t)\right) \Delta d\left(t, X_{0}(t, s)\right) h_{i}(t, s)\right. \\
& \left.-\partial_{t}^{\Gamma} h_{i}(t, s)\right) J^{0}(t, s) d s+\sum_{i=1}^{k} \varepsilon^{i} B_{i-1}^{C}(t)+\sum_{i=k+1}^{\infty} \varepsilon^{i} \bar{B}_{k, i}^{C}(t),
\end{aligned}
$$


where $B_{i-1}^{C}$ is the term of order $O\left(\varepsilon^{i}\right)$ determined from $d, \lambda_{0}, u_{j}, u_{j}^{ \pm}$and $h_{j}$, $0 \leq j \leq i-1$ and $\bar{B}_{k, i}^{C}$ denotes the term of order $O\left(\varepsilon^{i}\right)$ determined from $d, \lambda_{0}, u_{j}$, $u_{j}^{ \pm}, h_{j}, 0 \leq j \leq k$ for $i \geq k+1$.

Setting

$$
B_{k-1}=B_{k-1}^{A}+B_{k-2}^{B}+B_{k-1}^{C},
$$

and

$$
\bar{B}_{k, i}=\bar{B}_{k, i}^{A}+\bar{B}_{k, i}^{B}+\bar{B}_{k, i}^{C},
$$

we obtain

$$
\begin{aligned}
\int_{D}\left(\partial_{t} u_{k}^{\varepsilon}(t, x)-v(t)\right) d x & =2 \int_{\mathcal{S}}\left(\Delta d\left(t, X_{0}(t, s)\right)-\bar{\sigma} \lambda_{0}(t)-\frac{|D|}{2\left|\gamma_{t}^{v}\right|} v(t)\right) J^{0}(t, s) d s \\
& +2 \sum_{i=1}^{k} \varepsilon^{i} \int_{\mathcal{S}}\left(\left(\Delta d\left(t, X_{0}(t, s)\right)-\bar{\sigma} \lambda_{0}(t)\right) \Delta d\left(t, X_{0}(t, s)\right) h_{i}(t, s)\right. \\
& \left.-\partial_{t}^{\Gamma} h_{i}(t, s)\right) J^{0}(t, s) d s \\
& +\sum_{i=1}^{k} \varepsilon^{i} B_{i-1}+\sum_{i=k+1}^{\infty} \varepsilon^{i} \bar{B}_{k, i}-\left(B_{2}\right)-\left(C_{2}\right)+O\left(e^{-\zeta \delta / \varepsilon}\right),
\end{aligned}
$$

from (3.4), (3.6), (3.9), (3.10) and noting $\int_{\mathcal{S}} J^{0}(t, s) d s=\left|\gamma_{t}^{v}\right|$. Recalling that $\lambda_{0}(t)$ is defined as in (2.29), the first term of the right-hand side of (3.13) vanishes so that we can rewrite as

$$
\begin{aligned}
\int_{D}\left(\partial_{t} u_{k}^{\varepsilon}(t, x)-v(t)\right) d x \\
=\sum_{i=1}^{k} \varepsilon^{i}\left[2 \int _ { \mathcal { S } } \left(\left(\Delta d\left(t, X_{0}(t, s)\right)-\bar{\sigma} \lambda_{0}(t)\right) \Delta d\left(t, X_{0}(t, s)\right) h_{i}(t, s)\right.\right. \\
\quad-\bar{\sigma} \lambda_{i}(t)-b(t, s) h_{i}(t, s)+\frac{\bar{\sigma}}{2} \int_{\mathbb{R}} A^{i-1} m^{\prime}(\rho) d \rho \\
\left.\left.\quad+\bar{\sigma}\left(v(t)-\frac{b(t, s)}{2} \int_{\mathbb{R}} \rho\left(m^{\prime}(\rho)\right)^{2} d \rho\right) 1_{\{i=1\}}\right) J^{0}(t, s) d s+B_{i-1}\right] \\
\quad+\sum_{i=k+1}^{\infty} \varepsilon^{i} \bar{B}_{k, i}-\left(B_{2}\right)-\left(C_{2}\right)+O\left(e^{-\zeta \delta / \varepsilon}\right),
\end{aligned}
$$

by using (2.37) for $\partial_{t}^{\Gamma} h_{i}$ and noting $\int_{\mathcal{S}} \Delta^{\Gamma} h_{i}(t, s) J^{0}(t, s) d s=\int_{\gamma_{t}^{v}} \Delta^{\Gamma} h_{i}(t$, $\bar{s}) d \bar{s}=0$. We wrote $\Delta^{\Gamma}$ for the operator seen in the coordinate $s \in \mathcal{S}$, but we 
also write $\Delta^{\Gamma}$ for that defined in the coordinate $\bar{s}$ on $\gamma_{t}$. However, since $\lambda_{i}(t)$, $i=1, \ldots, k$ are defined as in (2.43), the first term of the right-hand side of (3.14) vanishes. This explains the reason that we determine $\lambda_{k}(t)$ as in (2.43) and we obtain

$$
\int_{D}\left(\partial_{t} u_{k}^{\varepsilon}(t, x)-v(t)\right) d x=\Phi_{k}^{\varepsilon}(t)
$$

where $\Phi_{k}^{\varepsilon}=\sum_{i=k+1}^{\infty} \varepsilon^{i} \bar{B}_{k, i}-\left(B_{2}\right)-\left(C_{2}\right)+O\left(e^{-\zeta \delta / \varepsilon}\right)$. Furthermore, note that each of $\left(B_{2}\right)$ and $\left(C_{2}\right)$ goes to 0 as $\varepsilon \downarrow 0$ exponentially fast due to the compatibility condition for $m^{\prime}, \partial_{\rho} u_{i}$ and $\partial_{t}^{\Gamma} u_{i}$.

\subsection{Estimates for $u_{k}$ and $u_{k}^{ \pm}$.}

3.3.1. Bounds for spatial derivatives of $b, X_{0}, d$ and $\mathbf{S}$. We introduce the following norms for $g \in C^{\infty}\left(\mathbb{R}_{+}\right)$.

Definition 3.1. For $k \in \mathbb{Z}_{+}$and $T>0$, we define $|g|_{k} \equiv|g|_{k, T}$ as

$$
|g|_{k, T}=\sum_{i=0}^{k} \sup _{t \in[0, T]}\left|\frac{d^{i} g}{d t^{i}}(t)\right|
$$

In the following, we take and fix a class $\mathcal{V}$ of functions $v \in C^{\infty}\left(\mathbb{R}_{+}\right)$and $T>0$ satisfying that

$$
C_{\mathcal{V}, T}=\max \left(C_{\mathcal{V}, T}^{(1)}, C_{\mathcal{V}, T}^{(2)}\right)<\infty
$$

where

$$
\begin{aligned}
C_{\mathcal{V}, T}^{(1)}:= & \sup _{\substack{v \in \mathcal{V}, s \in \mathcal{S}, r \in(-3 \delta, 3 \delta)}}\left\{\left|\partial^{\mathbf{m}} d\left(\cdot, X_{0}(\cdot, s)\right)\right|_{0, T},\left|\partial^{\mathbf{m}} S^{l}(\cdot, X(\cdot, r, s))\right|_{0, T}\right. \\
& \left.\left|\partial^{\mathbf{m}} X_{0}(\cdot, s)\right|_{0, T} ; 1 \leq l \leq n-1,|\mathbf{m}| \leq M\right\}<\infty
\end{aligned}
$$

and

$$
C_{\mathcal{V}, T}^{(2)}:=\sup _{v \in \mathcal{V}, s \in \mathcal{S}, t \in[0, T]}\left\{\left(\alpha_{-}(t, s)\right)^{-1},\left|\gamma_{t}^{v}\right|^{-1}\right\}<\infty .
$$

Here, in $C_{\mathcal{V}, T}^{(1)}, M=M(K) \in \mathbb{N}$ denotes the maximal number of the degrees of spatial derivatives taken over the terms appearing in $\widetilde{A}^{K}$ which is defined below (2.38) and $\delta>0$ is chosen as in Section 2.2. Recall Section 2.2 for $d, X_{0}, S^{l}$ and $X$ determined depending on $v$, and $\partial^{\mathbf{m}}=\partial_{x^{1}}^{m_{1}} \cdots \partial_{x^{n}}^{m_{n}},|\mathbf{m}|=\sum_{i=1}^{n} m_{i}$ for $m=$ $\left(m_{1}, \ldots, m_{n}\right) \in\left(\mathbb{Z}_{+}\right)^{n}$. Moreover, in $C_{\mathcal{V}, T}^{(2)}$,

$$
\alpha_{-}(t, s) \equiv \alpha_{-}^{v}(t, s):=\inf _{\xi \in \mathbb{R}^{n-1}:|\xi|=1}(\alpha(t, s) \xi, \xi)
$$


where $\alpha(t, s)=\left(\alpha_{i j}(t, s)\right)_{1 \leq i, j \leq n-1}$ is the matrix defined by (3.25) below, and $(\cdot, \cdot)$ and $|\cdot|$ denote the inner product and the norm of $\mathbb{R}^{n-1}$, respectively.

Later, for each $\omega \in \Omega$, we will take $\mathcal{V} \equiv \mathcal{V}(\omega):=\left\{\alpha \dot{w}^{\varepsilon} ; 0<\varepsilon \leq \varepsilon_{0}^{*}\right\}$. Then, for a.s. $\omega$, by Assumption 1.2, one can take $T=T(\omega):=\inf _{0<\varepsilon \leq \varepsilon_{0}^{*}} \sigma^{\varepsilon}>0$ such that equation (2.6) with $v=\alpha \dot{w}^{\varepsilon}$ has a solution up to the time $T(\omega)$ and $C_{\mathcal{V}}(\omega), T(\omega)<$ $\infty$ holds.

Recalling the definition (2.14) of $b(t, s)$, the constant $C_{\mathcal{V}, T}^{(1)}$ gives bounds for $b$. We need the bound $C_{\mathcal{V}, T}^{(2)}$ for deriving estimates on the fundamental solution (see (0.16) in [14] and Lemma 3.5) and on $\lambda_{0}(t)$ (see (0.22) in [14] and Proposition 3.6).

3.3.2. Bounds for time derivatives of $X_{0}, \partial^{\mathbf{m}} X_{0}$ and $\mathbf{S}$. Our goal is to give estimates for $u_{k}$ and $u_{k}^{ \pm}$; see Proposition 3.6 and Corollary 3.7 below. To do this, we will prepare several lemmas. The inward normal velocity $V$ is represented as $V(t, s)=\partial_{t} d\left(t, X_{0}(t, s)\right)$ from (2.12) and

$$
V(t, s)=\kappa(t, s)-\frac{1}{\left|\gamma_{t}^{v}\right|} \int_{\mathcal{S}} \kappa(t, s) J^{0}(t, s) d s+\frac{|D|}{2\left|\gamma_{t}^{v}\right|} v(t),
$$

holds with $\kappa(t . s)=\Delta d\left(t, X_{0}(t, s)\right)$ from (2.29) and (2.30). Let us formulate the following assumption on the time derivatives of $\partial^{\mathbf{m}} X_{0}$ and $\mathbf{S}$ by means of the norm of the forcing term $v$.

Assumption 3.1. There exist some $N=N(K) \in \mathbb{N}, T=T(\mathcal{V})>0$ and $C_{1}=C_{1}\left(C_{\mathcal{V}, T}, K, T\right)>0$ such that

$$
\sup _{1 \leq i \leq n-1} \sup _{r \in(-3 \delta, 3 \delta), s \in \mathcal{S}}\left|\partial_{t}^{k} \partial^{\mathbf{m}} S^{i}(\cdot, X(\cdot, r, s))\right|_{0, T} \leq C_{1}\left(1+|v|_{N, T}\right)^{N},
$$

for $k=0,1, \ldots, K,|\mathbf{m}| \leq M$ and $v \in \mathcal{V}$.

Under the choice $\mathcal{V} \equiv \mathcal{V}(\omega)=\left\{\alpha \dot{w}^{\varepsilon} ; 0<\varepsilon \leq \varepsilon_{0}^{*}\right\}$, Assumption 3.1 determines $\tau(\omega):=T(\mathcal{V}(\omega))$, up to which two bounds (3.21) and (3.22) hold. We will show in Section 5 that Assumption 3.1 is true for some $T=\tau(\omega)>0$ under a twodimensional setting as long as the limit curve $\gamma_{t}$ is convex.; see Lemma 5.1.

3.3.3. Schauder estimates for $h_{k}$. Recall that $h_{k}$ are the solutions of (2.44), which is rewritten as

$$
L h_{k}+\mathcal{L} h_{k}=F_{k-1},
$$


where $L=L_{t}$ is a differential operator defined by

$$
\begin{aligned}
L= & \partial_{t}^{\Gamma}-\Delta^{\Gamma}-b(t, s) \\
= & \left(\partial_{t}+\sum_{i=1}^{n-1} S_{t}^{i} \partial_{s^{i}}\right)-\left(\sum_{i=1}^{n-1} \Delta S^{i} \partial_{s^{i}}+\sum_{i, j=1}^{n-1} \nabla S^{i} \cdot \nabla S^{j} \partial_{s^{i} s^{j}}^{2}\right) \\
& -b(t, s) \\
= & \partial_{t}-\sum_{i, j=1}^{n-1} \alpha_{i j}(t, s) \partial_{s^{i} s^{j}}^{2}-\sum_{i=1}^{n-1} \beta_{i}(t, s) \partial_{s^{i}}-b(t, s),
\end{aligned}
$$

with

$$
\begin{aligned}
\alpha_{i j}(t, s) & =\nabla S^{i}(t) \cdot \nabla S^{j}(t), \beta_{i}(t, s)=-S_{t}^{i}(t)+\Delta S^{i}(t), \\
s & =\left(S^{1}(t), \ldots, S^{n-1}(t)\right) \in \mathcal{S},
\end{aligned}
$$

while $\mathcal{L}=\mathcal{L}_{t}$ is an integral operator acting on a function $u=u(s)$ defined by

$$
\begin{aligned}
\mathcal{L}_{t} u= & -\frac{1}{\left|\gamma_{t}^{v}\right|} \int_{\mathcal{S}}\left(\left(\Delta d\left(t, X_{0}\left(t, s^{\prime}\right)\right)-\bar{\sigma} \lambda_{0}(t)\right) \Delta d\left(t, X_{0}\left(t, s^{\prime}\right)\right)-b\left(t, s^{\prime}\right)\right) \\
& \times u\left(s^{\prime}\right) J^{0}\left(t, s^{\prime}\right) d s^{\prime},
\end{aligned}
$$

and $F_{k-1}$ denotes the right-hand side of (2.44). Since $v$ and the interface $\gamma_{t}^{v}$ are smooth, it follows that (3.23) with an initial condition $h_{k}(0, s)=0$ has a local unique solution for each $v$ from general argument for second-order parabolic partial differential equations, for example, by making use of Theorem 3.3.7 and Theorem 7.4.8 in [8] or Chapter V, Section 6 and Chapter VI, Section 4 in [20].

Our goal is to obtain estimates for the solutions $h_{k}$ of (2.44). To do this, we basically follow the argument given in Friedman [8] and derive the Schauder estimates. However, in our setting, the coefficients of the operators $L$ and $\mathcal{L}$ determined from $v=\alpha \dot{w}^{\varepsilon}$ are not bounded in $\varepsilon$, but controlled by the constant $C_{\mathcal{V}, T}$ and the norm $|v|_{1, T}$. We need to carefully study how the estimates for $h_{k}$ depend on these diverging factors. We first treat the contributions of the operator $L$; see Lemmas 3.2 and 3.3. The contribution of the operator $\mathcal{L}$ will be discussed starting from (3.33) below.

For $\tau \in[0, T]$ and $\xi \in \mathcal{S}$, let $Z(t, s ; \tau, \xi)$ be the fundamental solution of

$$
\left(\frac{\partial}{\partial t}-\sum_{i, j=1}^{n-1} \alpha_{i j}(\tau, \xi) \frac{\partial^{2}}{\partial s^{i} \partial s^{j}}\right) u(t, s)=0, \quad(t, s) \in(\tau, T] \times \mathcal{S},
$$

that is, $Z$ is a Gaussian kernel. Then the fundamental solution $\Gamma$ of $L_{t}$ is constructed by the usual parametrix method. Namely, by regarding $Z$ as the principal part of $\Gamma$, we can find $\Gamma$ in the form

$$
\Gamma(t, s ; \tau, \xi)=Z(t, s ; \tau, \xi)+\int_{\tau}^{t} \int_{\mathcal{S}} Z\left(t, s ; \sigma, s^{\prime}\right) \Phi\left(\sigma, s^{\prime} ; \tau, \xi\right) d \sigma d s^{\prime}
$$


where $\Phi$ is a function satisfying

$$
\Phi(t, s ; \tau, \xi)=L_{t} Z(t, s ; \tau, \xi)
$$

$$
+\int_{\tau}^{t} \int_{\mathcal{S}} L_{t} Z\left(t, s ; \sigma, s^{\prime}\right) \Phi\left(\sigma, s^{\prime} ; \tau, \xi\right) d \sigma d s^{\prime}
$$

Let $\mu \in\left(\frac{1}{2}, 1\right)$ and fix it. Then we have the following estimate on $\Gamma$.

LEMMA 3.1. The fundamental solution $\Gamma$ has the estimate:

$$
\begin{aligned}
|\Gamma(t, s ; \tau, \xi)| \leq & C_{8}\left(1+|v|_{N}\right)^{\nu_{0}+\frac{N}{1-\mu}} e^{C_{8}(T \vee 1)\left(1+|v|_{N}\right)^{\frac{N}{1-\mu}}} \\
& \times \frac{1}{(t-\tau)^{\frac{n-1}{2}}} e^{-C_{9} \frac{|s-\xi|^{2}}{(t-\tau)}}
\end{aligned}
$$

for $0 \leq \tau<t \leq T, s, \xi \in \mathcal{S}$ with some $\nu_{0} \in \mathbb{N}, C_{8}=C_{8}\left(C_{\mathcal{V}, T}, T, \mu\right)$ and $C_{9}=$ $C_{9}\left(C_{\mathcal{V}, T}, T, \mu\right)>0$.

Consider the following equation:

$$
\left\{\begin{array}{l}
L_{t} u(t, s)=f(t, s), \quad(t, s) \in(0, T] \times \mathcal{S}, \\
u(0, s)=u_{0}(s), \quad s \in \mathcal{S},
\end{array}\right.
$$

for $f \in \bar{C}^{\alpha}, \alpha \in(0,1]$ and $u_{0} \in C(\mathcal{S})$, where

$$
\begin{aligned}
& \bar{C}^{\alpha}=\left\{u(t, s)|| \overline{|u|}_{\alpha}<\infty\right\}, \\
& \overline{|u|}_{\alpha} \equiv{\overline{|u|_{\alpha, T}}}=|u|_{0}+\sup _{\substack{(t, s),(\bar{t}, \bar{s}) \\
\in[0, T] \times \mathcal{S}}} \frac{|u(t, s)-u(\bar{t}, \bar{s})|}{\sqrt{|s-\bar{s}|^{2}+|t-\bar{t}|}}, \\
&|u|_{0} \equiv|u|_{0, T}=\sup _{\substack{(t, s) \in[0, T] \times \mathcal{S} \\
\mid}}|u(t, s)|, \quad|s|=\left(\sum_{i=1}^{n-1}\left(s^{i}\right)^{2}\right)^{\frac{1}{2}} .
\end{aligned}
$$

Applying the argument of [8], Chapter 3, Section 3, Theorem 7 for our problem, we obtain the following lemma.

LEMMA 3.2. There exists a unique solution $u$ of (3.30) and $u \in \bar{C}^{2+\alpha}$, where

$$
\begin{aligned}
\bar{C}^{2+\alpha} & =\left\{u(t, s) \mid \overline{|u|}_{2+\alpha}<\infty\right\}, \\
\overline{|u|}_{2+\alpha} & =\overline{|u|}_{\alpha}+\sum_{|\mathbf{m}|=1} \overline{\left|\partial^{\mathbf{m}} u\right|_{\alpha}}+\sum_{|\mathbf{m}|=2} \overline{\left|\partial^{\mathbf{m}} u\right|_{\alpha}}+\sum{\overline{\left|\partial_{t} u\right|_{\alpha}}} .
\end{aligned}
$$

Furthermore, the following lemma is shown by using [8], Chapter 5, Section 3, Lemma 2. 
LEMMA 3.3. Let $u$ be the solution of (3.30). Then we have

$$
|u|_{0} \leq K_{1}\left(\sup _{s \in \mathcal{S}}\left|u_{0}(s)\right|+T \sup _{(t, s) \in[0, T] \times \mathcal{S}}|f(t, s)|\right),
$$

where

$$
K_{1} \equiv K_{1}(v)=C_{11} e^{2 C_{8}\left(1+|v|_{N}\right)^{p}(T \vee 1)},
$$

for some $C_{11}=C_{11}\left(C_{\mathcal{V}, T}, T, \mu\right)>0$ and $p=p\left(\mu, \nu_{0}\right) \in \mathbb{N}$.

We now study equation (3.23) for $h_{k}$ taking the contribution of the operator $\mathcal{L}_{t}$ into account. Let us consider

$$
\left\{\begin{array}{l}
L_{t} u(t, s)=g(t, s, u), \quad(t, s) \in(0, T] \times \mathcal{S}, \\
u(0, s)=0, \quad s \in \mathcal{S},
\end{array}\right.
$$

where

$$
g(t, s, u)=-\mathcal{L}_{t} u+F_{k-1}(t, s) .
$$

LEMMA 3.4. There exists a unique solution $u$ of (3.33) satisfying

$$
|u|_{0, T} \equiv \sup _{(t, s) \in[0, T] \times \mathcal{S}}|u(t, s)| \leq\left(C_{13} K_{3}\right)^{C_{13} K_{3}}\left|F_{k-1}\right|_{0, T},
$$

for some $C_{13}=C_{13}\left(C_{\mathcal{V}, T}, T, \mu\right)>0$ and

$$
K_{3} \equiv K_{3}(v)=e^{4 C_{8}\left(1+|v|_{N}\right)^{1+p}(T \vee 1)} .
$$

As for the regularity of the solution $u$ of (3.33), we have the following lemma. The proof is given based on the arguments in [8] or [21].

LEMMA 3.5. For the solution $u$ of (3.33) satisfying (3.35),

$$
|u|_{2+\alpha} \leq K_{4}\left(|u|_{0}+\left|F_{k-1}\right|_{\alpha}\right),
$$

holds for $K_{4} \equiv K_{4}(v)=C_{14}\left(1+|v|_{n_{0}(K)}\right)^{n_{0}(K)}$, where $C_{14}=C_{14}\left(C_{\mathcal{V}, T}, T, \mu\right)>0$ and $n_{0}=n_{0}(K) \in \mathbb{N}$.

3.3.4. Estimates for $u_{k}$ and $u_{k}^{ \pm}$. One can apply Lemma 3.5 for the solutions $h_{k}$ of (3.23) to obtain estimates for them. Based on this, we have the following estimates for $u_{k}$ and $u_{k}^{ \pm}$.

Proposition 3.6. For every $k=0,1, \ldots, K$,

$$
\sup _{(t, \rho, s) \in[0, T] \times \mathbb{R} \times \mathcal{S}}\left\{\left|u_{k}(t, \rho, s)\right|,\left|u_{k}^{ \pm}(t)\right|\right\} \leq\left(C_{15} K_{5}\right)^{C_{15} K_{5}},
$$

holds for some $C_{15}=C_{15}\left(C_{\mathcal{V}, T}, T, \mu\right)>0$ and

$$
K_{5} \equiv K_{5}(v)=e^{n_{1}(K)\left(1+|v|_{n_{1}(K)}\right)^{n_{1}(K)}(T \vee 1)},
$$

with some $n_{1}=n_{1}(K) \in \mathbb{N}$. 
PROOF. First, note that the estimates for $\left|\partial^{\mathbf{m}} X_{0}(\cdot, s)\right|_{0}$ and $\left|\partial^{\mathbf{m}} S^{j}(\cdot, x)\right|_{0}$ for every $|\mathbf{m}| \leq M$ are given by Assumption 3.1. Recalling that $u_{0}$ and $u_{0}^{ \pm}$are determined by (2.32) and (2.34), respectively, since we have $\left|\lambda_{0}(t)\right| \leq C \cdot C_{\mathcal{V}, T}(1+$ $|v|_{0, T}$ ) from (2.29) and $1 /\left|\gamma_{t}^{v}\right| \leq C_{\mathcal{V}, T}^{(2)}$ as we saw for (0.22) in the proof of Lemma 3.4 in [14], (3.37) is easily shown for $u_{0}$ and $u_{0}^{ \pm}$.

From this, one can derive the estimate for $F_{0}$ defined by the right-hand side of (2.44) with $k=1$, since $F_{0}$ contains $u_{0}, u_{0}^{ \pm}$and $b$. Thus, the estimate for $\left|h_{1}\right|_{0}$ is obtained by Lemma 3.4. In addition, the estimates for $\left|\partial_{t} h_{1}\right|_{0},\left|\partial_{s^{i}} h_{1}\right|_{0}$ and $\left|\partial_{s^{i} s^{j}}^{2} h_{1}\right|_{0}$ are obtained by Lemma 3.5. Similarly, the estimate for $\left|\partial^{\mathbf{m}} \partial_{t}^{l} F_{0}\right|_{0}$, $|\mathbf{m}| \leq M, l \leq K$ is obtained. Differentiating (3.23) and applying the above argument recursively, the estimates for $\left|\partial_{t}^{l} h_{1}\right|_{0}$ and $\left|\partial^{\mathbf{m}} h_{1}\right|_{0},|\mathbf{m}| \leq M, l \leq K$ are also obtained.

Recall that $u_{1}$ is represented by using $\widetilde{A}^{1}$, which contains $\lambda_{0}, u_{0}, h_{1}$. Therefore, the estimate for $\left|u_{1}\right|_{0}$ is obtained from those for $\lambda_{0}, u_{0}, h_{1}$. Once we have the estimate for $\left|u_{1}\right|_{0}$, similarly as above, the estimate for $\left|F_{1}\right|_{0}$ follows. From this, we have the estimate for $\left|h_{2}\right|_{0}$ by Assumption 3.1.

In this way, the estimates for $u_{k}, u_{k}^{ \pm}, k \leq K$ are obtained recursively by deriving the estimate for each term in $\widetilde{A}^{k}$. Since $K$ is finite and $\widetilde{A}^{k}, k \leq K$ contain only finitely many terms, it is easy to see that there exists a suitable $n_{1}(K)$ such that (3.37) holds. The proof is complete.

Finally in this section, we consider the random case taking $v(t)=\alpha \dot{w}^{\varepsilon}(t)$, where $w^{\varepsilon}(t)$ satisfies Assumption 1.1, and apply Proposition 3.6 for this case.

COROLlary 3.7. We assume Assumptions 1.1, 1.2 and 3.1, and define $G_{\varepsilon} \geq$ $e^{e}, 0<\varepsilon \leq 1$, from $H_{\varepsilon}$ appearing in Assumption 1.1 by the relation

$$
\log \log G_{\varepsilon}=H_{\varepsilon}^{2 n_{1}(K)},
$$

where $n_{1}(K) \in \mathbb{N}$ is the number determined by Proposition 3.6. Then we have

$$
\lim _{\varepsilon \downarrow 0} G_{\varepsilon}=\infty, \quad \lim _{\varepsilon \downarrow 0} \frac{G_{\varepsilon}}{|\log \varepsilon|}=0 .
$$

Furthermore, $u_{k}$ and $u_{k}^{ \pm}$determined from $v(t)=\alpha \dot{w}^{\varepsilon}(t)$ as above satisfy

$$
\sup _{(t, \rho, s) \in[0, T(\omega) \wedge \tau(\omega)] \times \mathbb{R} \times \mathcal{S}}\left\{\left|u_{k}(t, \rho, s)\right|,\left|u_{k}^{ \pm}(t)\right|\right\} \leq G_{\varepsilon}, \quad 0 \leq k \leq K,
$$

for every sufficiently small $\varepsilon>0$ and every $\omega \in \Omega$, where $T(\omega)>0$ is the minimum of that determined at the end of Section 3.3.1 and $\tau(\omega)$ given below Assumption 3.1 in Section 3.3.2.

Proof. The first one in (3.39) is clear from (3.38) and $\lim _{\varepsilon \downarrow 0} H_{\varepsilon}=\infty$. For the second one, use twice that $\lim _{\varepsilon \downarrow 0} \frac{\log a_{\varepsilon}}{\log b_{\varepsilon}}=0$ and $\lim _{\varepsilon \downarrow 0} b_{\varepsilon}=\infty$ imply 
$\lim _{\varepsilon \downarrow 0} \frac{a_{\varepsilon}}{b_{\varepsilon}}=0$. To show (3.40), we see from Assumption 1.1 that $v=\alpha \dot{w}^{\varepsilon}$ satisfies

$$
|v|_{n_{1}(K), T} \leq\left(n_{1}(K)+1\right) \alpha H_{\varepsilon} .
$$

From this, one can verify

$$
\left(C_{15} K_{5}\right)^{C_{15} K_{5}} \leq G_{\varepsilon}
$$

for every sufficiently small $\varepsilon>0$, where the left-hand side is the bound obtained in Proposition 3.6; note that $K_{5}=K_{5}(v)$ with $v=\alpha \dot{w}^{\varepsilon}$. Indeed,

$$
\log \log \left\{\left(C_{15} K_{5}\right)^{C_{15} K_{5}}\right\}=\log C_{15}+\log K_{5}+\log \log \left(C_{15} K_{5}\right),
$$

whose leading term for small $\varepsilon>0$ is $\log K_{5}$, and it has a bound

$$
\log K_{5}=n_{1}(K)\left(1+|v|_{n_{1}(K)}\right)^{n_{1}(K)}(T \vee 1) \leq C H_{\varepsilon}^{n_{1}(K)}<H_{\varepsilon}^{2 n_{1}(K)},
$$

with some $C>0$, by the definition of $K_{5}=K_{5}(v)$ and (3.41), for every $\varepsilon>0$ small enough, since $\lim _{\mathcal{L} \downarrow 0} H_{\varepsilon}=\infty$. Note that, recalling the remark $C_{\mathcal{V}}(\omega), T(\omega)<$ $\infty$ for $\mathcal{V}(\omega)=\left\{\alpha \dot{w}^{\varepsilon} ; 0<\varepsilon \leq \varepsilon_{0}^{*}\right\}$ made at the end of Section 3.3.1, $C_{15}=$ $C_{15}\left(C_{\mathcal{V}(\omega), T(\omega)}, T(\omega), \mu\right)$ is bounded in $\varepsilon$ so that the other terms in (3.43) are much smaller than $\log K_{5}$. Thus, we obtain (3.42) for every $\varepsilon>0$ small enough. Proposition 3.6 completes the proof.

\section{Proof of Theorem 1.1.}

4.1. The stochastic term $w^{\varepsilon}(t)$. This subsection gives two examples of $w^{\varepsilon}(t)$, which satisfy the condition (1.8) and Assumption 1.1. The integer $K$ is fixed throughout the paper.

The first example is a mollification of the Brownian motion with an extremely slow convergence speed. Let $w=w(t)$ be the one-dimensional standard Brownian motion and set

$$
\psi(\varepsilon)=(\log \log \log |\log \varepsilon|)^{\tilde{\beta}},
$$

with $\tilde{\beta}>0$. Let $W_{\varepsilon}(t), \varepsilon>0$ be the stopped process of $w$, that is, $W_{\varepsilon}(t)=$ $w(t \wedge \tau(\varepsilon))$, where $\tau(\varepsilon)$ is the first exit time of $w(t)$ from the interval $I_{\varepsilon}=$ $(-\psi(\varepsilon), \psi(\varepsilon))$, that is, $\tau(\varepsilon)=\inf \left\{t>0, w(t) \notin I_{\varepsilon}\right\}$. We define $w^{\varepsilon}(t)$ by

$$
w^{\varepsilon}(t)=\int_{0}^{\infty} \eta_{\psi(\varepsilon)}(t-s) W_{\varepsilon}(s) d s,
$$

where

$$
\eta_{\psi(\varepsilon)}(s)=\psi(\varepsilon) \eta(\psi(\varepsilon) s)
$$

and $\eta$ is a nonnegative $C^{\infty}$-function on $\mathbb{R}$, whose support is contained in $(0,1)$, satisfying $\int_{\mathbb{R}} \eta(u) d u=1$. 
LEMMA 4.1. For $w^{\varepsilon}(t)$ defined by (4.2), we have

$$
\left|\dot{w}^{\varepsilon}\right|_{k, T} \leq k|\eta|_{k+2} \psi(\varepsilon)^{k+2}, \quad k \in \mathbb{Z}_{+} .
$$

In particular, Assumption 1.1 holds for this $w^{\varepsilon}(t)$ with the choice of $H_{\varepsilon}=$ $n_{1}(K)|\eta|_{n_{1}(K)+2} \psi(\varepsilon)^{n_{1}(K)+2}$. The condition (1.8) holds obviously.

Indeed, a simple computation shows (4.3). Once (4.3) is shown, the rest of the lemma is obvious.

REMARK 4.1. If we choose $\tilde{\beta}>0$ as $2 \tilde{\beta} n_{1}(K)\left(n_{1}(K)+2\right)=1$, we have $H_{\varepsilon}^{2 n_{1}(K)}=\left(n_{1}(K)|\eta|_{n_{1}(K)+2}\right)^{2 n_{1}(K)} \log \log \log |\log \varepsilon|$. Since the condition (3.38) can be relaxed as $\log \log G_{\varepsilon}=C H_{\varepsilon}^{2 n_{1}(K)}$ with any constant $C>0$, one can choose $G_{\varepsilon}=\log |\log \varepsilon|$ for every small enough $\varepsilon>0$.

Let us give another example of $w^{\varepsilon}(t)$. Let $\xi=\xi(t), t \geq 0$ be a stochastic process satisfying the following conditions (see [11]):

1. $\xi$ is a stationary and strongly mixing stochastic process defined on a probability space $(\Omega, \mathcal{F}, P)$, that is, $\int_{0}^{\infty} \rho(t)^{\frac{1}{p}} d t<\infty$ for some $p>\frac{3}{2}$, where $\rho$ is given by

$$
\rho(t)=\sup _{s \geq 0} \sup _{A \in \mathcal{F}_{s+t, \infty}, B \in \mathcal{F}_{0, s}} \frac{|P(A \cap B)-P(A) P(B)|}{P(B)}, \quad t \geq 0,
$$

where $\mathcal{F}_{s, t}=\sigma(\xi(u), u \in[s, t])$.

2. $\xi(\cdot) \in C^{\infty}\left(\mathbb{R}_{+}\right)$, a.s. and $\left|\xi^{(k)}(t)\right| \leq M$, a.s., $k=0,1,2, \ldots, K$ for some nonrandom $M>0$.

3. $E[\xi(t)]=0$.

Let us define $\dot{w}^{\varepsilon}(t)$ as

$$
\dot{w}^{\varepsilon}(t)=A^{-1} \psi(\varepsilon) \xi\left(\psi(\varepsilon)^{2} t\right),
$$

where $\psi(\varepsilon)$ is taken as in (4.1) and $A=\left\{2 \int_{0}^{\infty} E[\xi(0) \xi(t)] d t\right\}^{1 / 2}$. Then, by a simple computation, we obtain the following lemma.

LEMMA 4.2. The process $\dot{w}^{\varepsilon}(t)$ defined by (4.4) satisfies

$$
\left|\dot{w}^{\varepsilon}\right|_{k, T} \leq \frac{M(k+1)}{A} \psi(\varepsilon)^{2 k+1}, \quad k \in \mathbb{Z}_{+} .
$$

In particular, Assumption 1.1 holds with the choice of $H_{\varepsilon}=M\left(n_{1}(K)+\right.$ 1) $\psi(\varepsilon)^{2 n_{1}(K)+1} / A$.

REMARK 4.2. The process $w^{\varepsilon}(t)$ converges to the Brownian motion $w(t)$ as $\varepsilon \downarrow 0$ in law; see [11]. Therefore, for the condition (1.8) in a.s.-sense, we need to apply Skorohod's representation theorem. 
4.2. Error estimate. In this subsection, coming back to the situation we discussed in Corollary 3.7, we estimate the error term $\delta_{k}^{\varepsilon}$ defined by

$$
\delta_{k}^{\varepsilon}(t, x):=\frac{\partial u_{k}^{\varepsilon}}{\partial t}-\Delta u_{k}^{\varepsilon}-\frac{1}{\varepsilon^{2}}\left(f\left(u_{k}^{\varepsilon}\right)-\varepsilon \lambda_{k}^{\varepsilon}\right)-v(t),
$$

with $v(t)=\alpha \dot{w}^{\varepsilon}(t)$. The corresponding quantity is introduced in [3], (9), but in our case, its bound involves an extra slowly diverging factor $G_{\varepsilon}$.

More precisely, in Sections 4.2-4.4, we assume a (nonrandom) $v(t)$ is given, and $u_{k}$ and $u_{k}^{ \pm}$determined from this $v(t)$ satisfy the bound (3.40) in Corollary 3.7 with $T(\omega)$ replaced by $T$ and for simplicity for every $\varepsilon>0$, that is,

$$
\sup _{(t, \rho, s) \in[0, T] \times \mathbb{R} \times \mathcal{S}}\left\{\left|u_{k}(t, \rho, s)\right|,\left|u_{k}^{ \pm}(t)\right|\right\} \leq G_{\varepsilon}, \quad 0 \leq k \leq K,
$$

holds for every $\varepsilon>0$. Later, we will apply the results obtained in these three sections for $v=\alpha \dot{w}^{\varepsilon}$ and $T=T(\omega)$.

Lemma 4.3. There exists $C_{\mathrm{err}}=C_{\mathrm{err}}(K, T)>0$ such that

$$
\sup _{(t, x) \in[0, T] \times D}\left|\delta_{k}^{\varepsilon}(t, x)\right| \leq \varepsilon^{k} C_{\mathrm{err}} G_{\varepsilon},
$$

holds for every $k=1,2, \ldots, K$ and $\varepsilon>0$.

We prepare another lemma; recall that $\Phi_{k}^{\varepsilon}(t)$ is defined just below (3.15).

Lemma 4.4. There exists $C_{\mathrm{err}}^{0}=C_{\mathrm{err}}^{0}(K, T)>0$ such that

$$
\left|\Phi_{k}^{\varepsilon}(t)\right|_{0, T} \leq \varepsilon^{k} C_{\mathrm{err}}^{0} G_{\varepsilon},
$$

for $k=0,1, \ldots, K$.

Indeed, recalling that $\bar{B}_{k, i}$ has a prefactor $\varepsilon^{k+1}$ and $\left(B_{2}\right),\left(C_{2}\right)$ decrease to 0 exponentially fast as $\varepsilon \downarrow 0$, the estimate (4.8) is obtained by the condition (4.6).

We may assume $C_{\mathrm{err}} \geq C_{\mathrm{err}}^{0}$ by taking $C_{\mathrm{err}} \vee C_{\mathrm{err}}^{0}$ for $C_{\mathrm{err}}$.

4.3. The Allen-Cahn operator. The goal of this subsection is to show Lemma 4.6, that is, the lower bound of the spectrum of the Allen-Cahn operator $-\varepsilon \Delta-$ $\varepsilon^{-1} f^{\prime}\left(v_{k}^{\varepsilon}\right)$, which is a linearization of the nonlinear Allen-Cahn equation around $v_{k}^{\varepsilon}$ defined by

$$
v_{k}^{\varepsilon}(t, x)=u_{k}^{\varepsilon}(t, x)-\frac{1}{|D|} \int_{0}^{t} \Phi_{k}^{\varepsilon}(s) d s, \quad 0 \leq k \leq K,
$$

for $x \in V_{3 \delta}^{t}$, where $\Phi_{k}^{\varepsilon}$ is the function defined as in (3.15).

An estimate similar to that in Lemma 4.6 is stated in [3], (10) (the condition $\int_{D} \phi d x=0$ is unnecessary), in which they consider the linearization around $u_{k}^{\varepsilon}$. 
The idea goes back to [4]. In our case, we need to take $v_{k}^{\varepsilon}$ instead of $u_{k}^{\varepsilon}$, since the vanishing condition $\int_{D}\left(u^{\varepsilon}(t, x)-v_{k}^{\varepsilon}(t, x)\right) d x=0$ holds under this choice because of the effect of $v(t)$, as we will see in Lemma 4.7 below. This vanishing condition is needed for Lemma 4.8, which is shown by applying Poincaré's inequality.

The argument to show Lemma 4.6 relies on [2], Section 2. Note that the correction term of $v_{k}^{\varepsilon}$ from $u_{k}^{\varepsilon}$ [i.e., the second term of (4.9)] is small, but it involves a slowly diverging factor $G_{\varepsilon}$ as is seen in Lemma 4.4. This gives the factor $G_{\varepsilon}$ in Lemma 4.6, differently from [2], Theorem 2.3.

We denote by $v_{k}^{\varepsilon}(t, \rho, s)$ the inner solution of $v_{k}^{\varepsilon}$ viewed under the coordinate $(t, \rho, s)$ defined by (2.19). By Lemma 4.4 and the condition (4.6), it follows that $v_{k}^{\varepsilon}, 2 \leq k \leq K$ is rewritten into

$$
v_{k}^{\varepsilon}(t, \rho, s)=m(\rho)-\varepsilon \lambda_{0}(t) \theta_{1}(\rho)+\varepsilon^{2} q^{\varepsilon}(t, \rho, s),
$$

where $\theta_{1}(\rho)$ is the function given below $(2.31)$ and $q^{\varepsilon}(t, \rho, s)$ is a function satisfying

$$
\sup _{\rho \in \mathbb{R}} \sup _{s \in \mathcal{S}} \sup _{\varepsilon \in(0,1]}\left|q^{\varepsilon}(\cdot, \rho, s)\right|_{0} \leq C_{\mathrm{err}} G_{\varepsilon} .
$$

For measurable and integrable functions $\Psi(t, z, s), \Phi(t, z, s), \psi(t, r, s)$ and $\phi(t, r, s), z \in I_{\varepsilon} \subset \mathbb{R}, r \in I_{1}, s \in \mathcal{S}, t \in[0, T]$, by following Section 2 in [2], we define

$$
\begin{aligned}
L^{s}\langle\Psi, \Phi\rangle & =\int_{I_{\varepsilon}}\left(\Psi_{z} \Phi_{z}-f^{\prime}\left(v_{k}^{\varepsilon}(t, \varepsilon z, s)\right) \Psi \Phi\right) J(t, \varepsilon z, s) d z, \\
L^{s}(\psi, \phi) & =\int_{I_{1}}\left(\varepsilon \psi_{r} \phi_{r}-\varepsilon^{-1} f^{\prime}\left(v_{k}^{\varepsilon}(t, r, s)\right) \psi \phi\right) J(t, r, s) d r, \\
L^{0}\langle\Psi, \Phi\rangle & =\int_{I_{\varepsilon}}\left(\Psi_{z} \Phi_{z}-f^{\prime}(m(z)) \Psi \Phi\right) d z, \\
\langle\Psi, \Phi\rangle & =\int_{I_{\varepsilon}} \Psi \Phi d z, \quad\langle\Psi, \Phi\rangle_{s}=\int_{I_{\varepsilon}} \Psi \Phi J(t, \varepsilon z, s) d z, \\
\|\Psi\| & =\langle\Psi, \Psi\rangle^{\frac{1}{2}}, \quad\|\Psi\|_{s}=\langle\Psi, \Psi\rangle_{s}^{\frac{1}{2}}, \\
(\psi, \phi)_{s} & =\int_{I_{1}} \psi \phi J(t, r, s) d r, \quad|\psi|_{s}=(\psi, \psi)_{s}^{\frac{1}{2}},
\end{aligned}
$$

where $\Psi_{z}(t, z, s), \psi_{r}(t, r, s)$ represents $\frac{\partial \Psi}{\partial z}(t, z, s), \frac{\partial \psi}{\partial r}(t, r, s)$, respectively, $z=$ $\frac{r}{\varepsilon}, J(t, r, s)$ is given by (3.5) and $I_{\varepsilon}=\left(-\frac{1}{\varepsilon}, \frac{1}{\varepsilon}\right)$. Note that (4.12) stands for the quadratic form corresponding to the operator $-\Delta-f^{\prime}\left(v_{k}^{\varepsilon}\right)$ in the microscopic view point, while (4.13) represents that in the macroscopic view point. Under these settings, the following lemma holds. 
LEMMA 4.5. Let us set $\bar{\lambda}_{1}(t, s)=\inf _{\|\Psi\|_{s}=1} L^{s}\langle\Psi, \Psi\rangle, s \in \mathcal{S}, t \in[0, T]$. Then there exist constants $c_{1}, c_{2}>0$ depending on $K, T$ and $\varepsilon_{0,1}=\varepsilon_{0,1}(K, T)>0$ such that

$$
-c_{1} \varepsilon^{2} C_{\mathrm{err}} G_{\varepsilon} \leq \bar{\lambda}_{1}(t, s) \leq c_{2} \varepsilon^{2} C_{\mathrm{err}} G_{\varepsilon}
$$

holds for every $\varepsilon \in\left(0, \varepsilon_{0,1}\right]$ and $t \in[0, T]$.

From Lemma 4.5 and [2], Theorem 2.3, in which the lower bound of the quadratic form (4.13) is studied, we obtain the following lemma.

LEMMA 4.6. There exists a constant $C_{A}>0$ independent of $\varepsilon$ and $t$ such that for every $\varepsilon \in\left(0, \varepsilon_{0,1}\right]$ and $\psi=\psi(t, \cdot) \in H^{1}(D), t \in[0, T]$,

$$
\begin{aligned}
& \int_{D}\left(\varepsilon|\nabla \psi(t, x)|^{2}-\varepsilon^{-1} f^{\prime}\left(v_{k}^{\varepsilon}(t, x)\right) \psi(t, x)^{2}\right) d x \\
& \geq-C_{A} \varepsilon C_{\mathrm{err}} G_{\varepsilon} \int_{D} \psi(t, x)^{2} d x, \quad 0 \leq t \leq T,
\end{aligned}
$$

holds.

4.4. Estimate for the difference between $u_{K}^{\varepsilon}$ and $u^{\varepsilon}$. Similar to [3], (12), we take initial data $g^{\varepsilon}=g^{\varepsilon}(x)$ of (1.1) or (2.5) satisfying the following three conditions:

$$
\begin{aligned}
g^{\varepsilon}(x) & =u_{K}^{\varepsilon}(0, x)+\phi^{\varepsilon}(x), \\
\left\|\phi^{\varepsilon}\right\|_{L^{2}(D)} & \leq C_{1}^{-\frac{1}{p}} \varepsilon^{K}, \\
\int_{D} \phi^{\varepsilon}(x) d x & =0,
\end{aligned}
$$

for sufficiently small $\varepsilon>0$, where $C_{1}>0$ is the constant appearing in (4.25) in the proof of Theorem 4.9 below and $p=\min \left\{\frac{4}{n}, 1\right\}$ is the number which will be given in Lemma 4.8. Recall that $u_{K}^{\varepsilon}(0, x)$ is defined by (3.2) with $t=0$ and $K>$ $\max (n+2,6)$ is fixed throughout the paper. Then the following lemma is shown by an elementary computation.

LEMMA 4.7. Let $u^{\varepsilon}$ be the solution of (2.5) with initial data $g^{\varepsilon}$ satisfying the conditions (4.19) and (4.21). Then, $\int_{D} R(t, x) d x=0$ holds, where $R(t, x)=$ $u^{\varepsilon}(t, x)-v_{K}^{\varepsilon}(t, x)$.

The next lemma is taken from [3], page 530; see page 547 and middle of page 530 for the proof. 
Lemma 4.8. Let $D \subset \mathbb{R}^{n}$ be a bounded domain. Let $p=\min \left\{\frac{4}{n}, 1\right\}$. Then there exists $C_{n}(D)>0$ such that for every $R \in H^{1}(D)$ with $\int_{D} R(x) d x=0$,

$$
\|R\|_{L^{2+p}(D)}^{2+p} \leq C_{n}(D)\|R\|_{L^{2}(D)}^{p}\|\nabla R\|_{L^{2}(D)}^{2},
$$

holds. Furthermore, there exists a constant $C^{\prime}>0$ such that

$$
R\left(f(u+R)-f(u)-f^{\prime}(u) R\right) \leq C^{\prime}|R|^{2+p},
$$

for every $|u| \leq 2$ and $R \in \mathbb{R}$.

The following theorem extends [3], Lemma 2, in our setting. Note that the difference between $v_{K}^{\varepsilon}$ and $u_{K}^{\varepsilon}$ is small; recall (4.9) and Lemma 4.4.

THEOREM 4.9. Let $u^{\varepsilon}$ be the solution considered in Lemma 4.7 and assume (4.19)-(4.21) for the initial data $g^{\varepsilon}$. Then, for sufficiently small $\varepsilon \in\left(0, \varepsilon_{1}\right]$, where $\varepsilon_{1}=\min \left(\varepsilon_{0,1}, e^{-1}\right)$,

$$
\sup _{t \in[0, T]}\left\|v_{K}^{\varepsilon}(t)-u^{\varepsilon}(t)\right\|_{L^{2}(D)} \leq C_{2} \varepsilon^{K-1}|\log \varepsilon|,
$$

holds for some constant $C_{2}=C_{2}\left(D, C_{A}\right)>0$.

ProOF. From (3.39), we can assume

$$
C_{1}^{-\frac{1}{p}} \leq T|D|^{\frac{1}{2}} C_{\mathrm{err}} G_{\varepsilon} \quad \text { and } \quad \frac{2 C_{A} C_{\mathrm{err}} G_{\varepsilon} T}{|\log \varepsilon|} \leq 1,
$$

for sufficiently small $\varepsilon>0$, where the constant $C_{1}=C_{n}(D) C^{\prime}>0$ is determined from the two constants given in Lemma 4.8. We can also assume

$$
\varepsilon|\log \varepsilon||D|^{\frac{1}{2}} C_{A}^{-1} C_{1}^{\frac{1}{p}} \leq 1
$$

for sufficiently small $\varepsilon>0$.

Similar to the proof of Lemma 2 in [3], using (4.23) with $R=u^{\varepsilon}-v_{K}^{\varepsilon}$ and $u=v_{K}^{\varepsilon}$, we obtain

$$
\begin{aligned}
\frac{1}{2} \frac{d}{d t} \| & R(t) \|_{L^{2}(D)}^{2}+\int_{D}\left(|\nabla R(t, x)|^{2}-\varepsilon^{-2} f^{\prime}\left(v_{K}^{\varepsilon}\right) R^{2}(t, x)\right) d x \\
\leq & \int_{D}\left(C^{\prime} \varepsilon^{-2}|R(t, x)|^{2+p}+\left|R(t, x) \delta_{K}^{\varepsilon}(t, x)\right|\right) d x \\
\leq & \int_{D} C^{\prime} \varepsilon^{-2}|R(t, x)|^{2+p} d x \\
& +\|R(t)\|_{L^{2}(D)}\left\|\delta_{K}^{\varepsilon}(t)\right\|_{L^{2}(D)}, \quad t \in[0, T] .
\end{aligned}
$$

Note that $\left|v_{K}^{\varepsilon}\right| \leq 2$ holds for sufficiently small $\varepsilon>0$ because of the definition (4.9) of $v_{K}^{\varepsilon}$, the construction of $u_{K}^{\varepsilon}$, the condition (4.6) and Lemma 4.4 for $\Phi_{K}^{\varepsilon}(t)$. 
However, the second term in the left-hand side of (4.27) can be decomposed and be bounded from below as follows for all $\varepsilon \in\left(0, \varepsilon_{1}\right]$ :

$$
\begin{aligned}
& \varepsilon^{2} \int_{D}\left(|\nabla R(t, x)|^{2}-\varepsilon^{-2} f^{\prime}\left(v_{K}^{\varepsilon}\right) R^{2}(t, x)\right) d x \\
& +\left(1-\varepsilon^{2}\right) \int_{D}\left(|\nabla R(t, x)|^{2}-\varepsilon^{-2} f^{\prime}\left(v_{K}^{\varepsilon}\right) R^{2}(t, x)\right) d x \\
& \quad \geq \varepsilon^{2}\|\nabla R(t)\|_{L^{2}(D)}^{2}-\bar{c}_{1}\|R(t)\|_{L^{2}(D)}^{2}-C_{A} C_{\mathrm{err}} G_{\varepsilon}\|R(t)\|_{L^{2}(D)}^{2}
\end{aligned}
$$

Here, for the first term, we have used the assumption (iii) for $f: f^{\prime}\left(v_{K}^{\varepsilon}(t, x)\right) \leq \bar{c}_{1}$, while for the second term, we have applied Lemma 4.6 with $\psi(t, x)=R(t, x)$ and omitted the factor $\left(1-\varepsilon^{2}\right)$. On the other hand, for the first term in the right-hand side of (4.27), we can apply the interpolation inequality (4.22) and finally obtain

$$
\begin{aligned}
\frac{1}{2} \frac{d}{d t}\|R(t)\|_{L^{2}(D)}^{2} \leq & \left\|\delta_{K}^{\varepsilon}(t)\right\|_{L^{2}(D)}\|R(t)\|_{L^{2}(D)} \\
& +\left(\bar{c}_{1}+C_{A} C_{\mathrm{err}} G_{\varepsilon}\right)\|R(t)\|_{L^{2}(D)}^{2} \\
& -\varepsilon^{2}\|\nabla R(t)\|_{L^{2}(D)}^{2}\left(1-C_{1} \varepsilon^{-4}\|R(t)\|_{L^{2}(D)}^{p}\right)
\end{aligned}
$$

for $t \in[0, T]$, where $C_{1}>0$ is defined below (4.25).

Now, consider the time $T_{\varepsilon} \geq 0$ defined by

$$
T_{\varepsilon}:=\inf \left\{t \geq 0 ;\|R(t)\|_{L^{2}(D)} \geq C_{1}^{-\frac{1}{p}} \varepsilon^{\frac{4}{p}}\right\} \wedge T .
$$

If the above set $\{\cdot\}$ is empty, we define $T_{\varepsilon}=T$. The goal is to show $T_{\varepsilon}=T$ and the conclusion (4.24) based on this. From (4.19) and (4.20), we have $R(0)=\phi^{\varepsilon}$ and

$$
\|R(0)\|_{L^{2}(D)} \leq C_{1}^{-\frac{1}{p}} \varepsilon^{K},
$$

for all $0<\varepsilon \leq \varepsilon_{1}$, which implies that $T_{\varepsilon}>0$ since $K>\frac{4}{p}+2$. Note that $K>\frac{4}{p}+2$ follows from $K>\max (n+2,6)$ and the choice of $p: p=\min \left\{\frac{4}{n}, 1\right\}$. On the other hand, from (4.28) and the definition of $T_{\varepsilon}$, which guarantees the nonpositivity of the last term in (4.28) for $t \leq T_{\varepsilon}$, we obtain that for every $t \in\left[0, T_{\varepsilon}\right]$,

$$
\frac{d}{d t}\|R(t)\|_{L^{2}(D)} \leq 2 C_{A} C_{\mathrm{err}} G_{\varepsilon}\|R(t)\|_{L^{2}(D)}+\left\|\delta_{K}^{\varepsilon}(t)\right\|_{L^{2}(D)}
$$

where we have estimated $\bar{c}_{1}$ as $\bar{c}_{1} \leq C_{A} C_{\mathrm{err}} G_{\varepsilon}$ for sufficiently small $\varepsilon>0$ from (3.39). Then Gronwall's lemma shows that

$$
\sup _{t \in\left[0, T_{\varepsilon}\right]}\|R(t)\|_{L^{2}(D)} \leq e^{2 C_{A} C_{\mathrm{err}} G_{\varepsilon} T_{\varepsilon}}\left(\|R(0)\|_{L^{2}(D)}+\int_{0}^{T_{\varepsilon}}\left\|\delta_{K}^{\varepsilon}(t)\right\|_{L^{2}(D)} d t\right) .
$$

However, Lemma 4.3 implies

$$
\int_{0}^{T_{\varepsilon}}\left\|\delta_{K}^{\varepsilon}(t)\right\|_{L^{2}(D)} d t \leq T_{\varepsilon} \varepsilon^{K}|D|^{\frac{1}{2}} C_{\mathrm{err}} G_{\varepsilon}
$$


and, therefore, noting $e^{2 C_{A} C_{\mathrm{err}} G_{\varepsilon} T_{\varepsilon}} \leq e^{|\log \varepsilon|}=\varepsilon^{-1}$ from (4.25), we obtain

$$
\begin{aligned}
\sup _{t \in\left[0, T_{\varepsilon}\right]}\|R(t)\|_{L^{2}(D)} & \leq 2 \varepsilon^{K-1} T|D|^{\frac{1}{2}} C_{\mathrm{err}} G_{\varepsilon} \\
& \leq \varepsilon^{K-1}|\log \varepsilon||D|^{\frac{1}{2}} C_{A}^{-1}<\varepsilon^{\frac{4}{p}} C_{1}^{-\frac{1}{p}},
\end{aligned}
$$

where we have used (4.29), (4.30), (4.25) and $T_{\varepsilon} \leq T$ for the first inequality, (3.39) for the second for sufficiently small $\varepsilon>0$ and then $K>\frac{4}{p}+2$ and (4.26) for the last one. Thus, $T_{\varepsilon}=T$ holds, which implies that we obtain (4.24) by setting $C_{2}=|D|^{\frac{1}{2}} C_{A}^{-1}$. The proof is complete.

4.5. Proof of Theorem 1.1. Let $\gamma_{0}$ be a smooth hypersurface in $D$ satisfying the conditions in Theorem 1.1.

DEFINITION 4.1. The solution of (1.4) with an initial hypersurface $\gamma_{0}$ means the hypersurface $\Gamma=\bigcup_{0 \leq t<\sigma}\left(\gamma_{t} \times\{t\}\right)$ and $(d(t \wedge \sigma), w(t \wedge \sigma)) \in C^{2}(\mathcal{O}) \times \mathbb{R}$, $t \geq 0$, with a stopping time $\sigma$ defined on a probability space $(\Omega, \mathcal{F}, P)$ equipped with the filtration $\left(\mathcal{F}_{t}\right)_{t \geq 0}$ and $\mathcal{O}$ being an open neighborhood of $\gamma_{0}$ (cf. [26]) such that:

(i) $d(t, x)$ is an $\left(\mathcal{F}_{t}\right)$-adapted signed distance of $x \in \mathcal{O}$ to $\gamma_{t}$ for $t \in[0, \sigma)$ and satisfies $|\nabla d|=1$.

(ii) $w(t)$ is an $\left(\mathcal{F}_{t}\right)$-Brownian motion.

(iii) The following stochastic integral equation holds in Stratonovich sense:

$$
\begin{aligned}
d(t \wedge \sigma, x)= & d(0, x)+\int_{0}^{t \wedge \sigma} g\left(D^{2} d(u, x), d(u, x)\right) d u \\
& -\int_{0}^{t \wedge \sigma} \frac{d u}{\left|\gamma_{u}\right|} \int_{\gamma_{u}} \Delta d(u, \bar{s}) d \bar{s} \\
& +\int_{0}^{t \wedge \sigma} \frac{\alpha|D|}{2\left|\gamma_{u}\right|} \circ d w(u), \quad x \in \mathcal{O} .
\end{aligned}
$$

Here, $D^{2} d$ denotes the Hessian of $d$ and $g(A, q)=\operatorname{tr}\left(A(I-q A)^{-1}\right)$ for a symmetric matrix $A$ and $q \in \mathbb{R}$.

It follows from Assumption 1.2 that the solution $\left(\gamma_{t}^{\varepsilon}, d^{\varepsilon}\right) \equiv\left(\gamma_{t}^{\alpha \dot{w}^{\varepsilon}}, d^{\alpha \dot{w}^{\varepsilon}}\right)$ of (2.6) with $v=\alpha \dot{w}^{\varepsilon}$ exists uniquely for each $0<\varepsilon \leq 1$ and, furthermore, a unique solution $\Gamma=\bigcup_{0 \leq t<\sigma}\left(\gamma_{t} \times\{t\}\right)$ and $(d(t \wedge \sigma), w(t \wedge \sigma))$ of (1.4) with an initial hypersurface $\gamma_{0}$ also exists.

We are now in the position to give the proof of the main theorem of this paper.

ProOF of TheOREM 1.1. We assume Assumptions 1.1, 1.2 and 3.1, take initial data $g^{\varepsilon}$ of (1.1) satisfying the three conditions (4.19)-(4.21) and fix $T>0$ as 
in Assumption 3.1. Note that the solution of (1.1) exists uniquely for $t \in[0, T]$ a.s., since $\dot{w}^{\varepsilon}$ is $C^{\infty}$ in $t$. By Corollary 3.7, the condition (4.6) holds for $v=\alpha \dot{w}^{\varepsilon}$ and $T=T(\omega) \wedge \tau(\omega)$; recall that $T(\omega)$ determined from $\sigma^{\varepsilon}$ in Section 3.3.1 satisfies $T(\omega) \equiv T^{\varepsilon_{0}^{*}}(\omega) \uparrow \sigma(\omega)$ as $\varepsilon_{0}^{*} \downarrow 0$ a.s. and $\tau(\omega)$ is given below Assumption 3.1. Therefore, by (4.9), Lemma 4.4 and recalling the construction of $u_{K}^{\varepsilon}$, we see that

$$
\lim _{\varepsilon \downarrow 0} \sup _{t \in[0, T(\omega) \wedge \tau(\omega) \wedge T]}\left\|v_{K}^{\varepsilon}(t, \cdot)-m\left(d^{\varepsilon}(t, \cdot) / \varepsilon\right)\right\|_{L^{\infty}(D)}=0 \quad \text { a.s. }
$$

Furthermore, it follows from Assumption 1.2 that

$$
\lim _{\varepsilon \downarrow 0} \sup _{t \in[0, T]}\left\|m\left(d^{\varepsilon}\left(t \wedge \sigma^{\varepsilon}, \cdot\right) / \varepsilon\right)-\chi_{\gamma_{t \wedge \sigma}}(\cdot)\right\|_{L^{2}(D)}=0 \quad \text { a.s. }
$$

Moreover, noting Corollary 3.7 again, Theorem 4.9 implies

$$
\sup _{t \in[0, T(\omega) \wedge \tau(\omega) \wedge T]}\left\|u^{\varepsilon}(t, \cdot)-v_{K}^{\varepsilon}(t, \cdot)\right\|_{L^{2}(D)} \leq C_{2} \varepsilon^{K-1}|\log \varepsilon| \quad \text { a.s. }
$$

From (4.32)-(4.34), we obtain

$$
\lim _{\varepsilon \downarrow 0} \sup _{t \in \mathbb{R}_{+}}\left\|u^{\varepsilon}\left(t \wedge \sigma^{\varepsilon} \wedge \tau \wedge T, \cdot\right)-\chi_{\gamma_{t \wedge \sigma \wedge \tau \wedge T}}(\cdot)\right\|_{L^{2}(D)}=0 \quad \text { a.s. }
$$

The proof is completed, since $T=T^{\varepsilon_{0}^{*}}(\omega) \uparrow \sigma(\omega)$ as $\varepsilon_{0}^{*} \downarrow 0$.

5. Local existence and uniqueness for the limit dynamics (1.4). In this section, we consider the stochastically perturbed volume preserving mean curvature flow (1.4), which appears in the limit. We write $c=|D| / 2$ for simplicity. As explained in Section 1, the stochastic term destroys the volume conservation law. Here, we restrict ourselves in two-dimension and discuss under the situation that the closed curve $\gamma_{t}$ stays strictly convex. We prove the local existence and uniqueness of the stochastic evolution governed by (1.4) by extending the method employed in [11], and show that Assumption 3.1 holds up to some stopping time $T=\tau(\omega)>0$ assuming Assumption 1.2 in a.s.-sense and then prove Assumption 1.2 in the sense of law for $\dot{w}^{\varepsilon}$ given by (4.4). The difference between a.s.sense and law-sense can be filled by applying Skorohod's theorem and changing the probability space as we pointed out in Section 1.

A strictly convex closed plane curve $\gamma$ can be parameterized by $\theta \in S:=[0,2 \pi)$ in terms of the Gauss map, that is, the position $X_{0}$ on $\gamma$ is denoted by $X_{0}(\theta)$ if the angle between one fixed direction $\mathbf{e}:=(1,0)$ in the plane $\mathbb{R}^{2}$ and the outward normal $\vec{n}\left(X_{0}\right)$ at $X_{0}$ to $\gamma$ is $\theta$. The set $S$ or a unit circle in $\mathbb{R}^{2}$ plays a role of the reference manifold $\mathcal{S}$. We further denote by $\kappa=\kappa(\theta)>0$ the curvature of $\gamma$ at $X_{0}=X_{0}(\theta)$. Under these notation, the dynamics (1.4) is rewritten into the stochastic integro-differential equation for $\kappa=\kappa(t, \theta)$ :

$$
\frac{\partial \kappa}{\partial t}=\kappa^{2} \frac{\partial^{2} \kappa}{\partial \theta^{2}}+\kappa^{3}-\kappa^{2} \cdot \bar{\kappa}+\frac{c \alpha \kappa^{2}}{|\gamma|} \circ \dot{w}(t),
$$


where $\bar{\kappa}$ denotes the average of $\kappa$ over the curve $\gamma=\gamma_{t}$ and $|\gamma|$ stands for the length of $\gamma$; heuristically (5.1) is derived by applying [16], page 17, (2.20) with $V=\kappa-\bar{\kappa}+\frac{c \alpha}{|\gamma|} \dot{w}(t)$ and see also [11]. The volume (length) element $d \bar{\theta}$ on $\gamma$ is given by $d \bar{\theta}=\left|\partial_{\theta} X_{0}(\theta)\right| d \theta$. Since $X_{0}(\theta) \in \mathbb{R}^{2} \cong \mathbb{C}$ is written as

$$
X_{0}(\theta)=X_{0}(0)-\sqrt{-1} \int_{0}^{\theta} \frac{e^{\sqrt{-1} \theta^{\prime}}}{\kappa\left(\theta^{\prime}\right)} d \theta^{\prime},
$$

we see that $\left|\partial_{\theta} X_{0}(\theta)\right|=1 / \kappa(\theta)$. Therefore, $\bar{\kappa}$ and $|\gamma|$ are given by

$$
\begin{aligned}
|\gamma| & :=\int_{S}\left|\partial_{\theta} X_{0}(\theta)\right| d \theta=\int_{S} \frac{d \theta}{\kappa(\theta)}, \\
\bar{\kappa} & :=\frac{1}{|\gamma|} \int_{S} \kappa(\theta)\left|\partial_{\theta} X_{0}(\theta)\right| d \theta=\frac{2 \pi}{|\gamma|}=2 \pi\left(\int_{S} \frac{d \theta}{\kappa(\theta)}\right)^{-1},
\end{aligned}
$$

respectively, which are functionals of $\kappa=\{\kappa(\theta) ; \theta \in S\}$.

As in [11], we introduce a cut-off because of the singularity in (5.1). For $L \in \mathbb{N}$, we define a cut-off function $\chi_{L} \in C_{b}^{\infty}(\mathbb{R})$ (in particular, $\chi_{L}, \chi_{L}^{\prime}, \ldots$ are all bounded) such that $\chi_{L}(x)=x$ for $x \in[1 / L, L]$ and $1 / 2 L \leq \chi_{L}(x) \leq 2 L$ for all $x \in \mathbb{R}$, and set

$$
\begin{aligned}
|\gamma|_{L} & \equiv|\gamma|_{L}(\kappa(\cdot))=\int_{S} \frac{d \theta}{\chi_{L}(\kappa(\theta))}, \\
a_{L}(\kappa) & =\chi_{L}^{2}(\kappa), \\
b_{L}(\theta, \kappa(\cdot)) & =\chi_{L}^{3}(\kappa(\theta))-2 \pi \chi_{L}^{2}(\kappa(\theta))|\gamma|_{L}^{-1}, \\
h_{L}(\theta, \kappa(\cdot)) & =c \alpha \chi_{L}^{2}(\kappa(\theta))|\gamma|_{L}^{-1} .
\end{aligned}
$$

Fixing $L$ for a while and denoting $a_{L}, b_{L}, h_{L}$ by $a, b, h$ for simplicity, we consider the stochastic integro-differential equation with cut-off for $\kappa=\kappa(t, \theta)$ :

$$
\frac{\partial \kappa}{\partial t}=a(\kappa) \frac{\partial^{2} \kappa}{\partial \theta^{2}}+b(\cdot, \kappa)+h(\cdot, \kappa) \circ \dot{w}(t), \quad t>0, \theta \in S .
$$

We also consider the dynamics (2.6) with $v(t)=\alpha \dot{w}^{\varepsilon}(t)$, which is described by the integro-differential equation:

$$
\frac{\partial \kappa}{\partial t}=a(\kappa) \frac{\partial^{2} \kappa}{\partial \theta^{2}}+b(\cdot, \kappa)+h(\cdot, \kappa) \dot{w}^{\varepsilon}(t), \quad t>0, \theta \in S,
$$

by replacing $\dot{w}(t)$ in (5.5) with $\dot{w}^{\varepsilon}(t)$. We gave two examples of smooth noises $\dot{w}^{\varepsilon}(t)$ in Section 4.1. Since $\dot{w}^{\varepsilon}(t)$ is smooth in $t$, (5.6) has a unique solution for every $\omega$.

We now show that Assumption 3.1 holds with the choice $\mathcal{V}=\left\{\alpha \dot{w}^{\varepsilon} ; 0<\varepsilon \leq\right.$ $\left.\varepsilon_{0}^{*}\right\}$ in the setting of this section and assuming Assumption 1.2 in a.s.-sense. The 
dynamics (2.6) is rewritten into the integro-differential equation for $\kappa=\kappa(t, \theta)$ :

$$
\frac{\partial \kappa}{\partial t}=\kappa^{2} \frac{\partial^{2} \kappa}{\partial \theta^{2}}+\kappa^{3}-\kappa^{2} \cdot \bar{\kappa}+\frac{c \kappa^{2}}{\left|\gamma^{v}\right|} v,
$$

where the averaged curvature $\bar{\kappa}$ and the length $\left|\gamma^{v}\right|$ are determined from $\kappa=\kappa(t)$ by (5.3) and (5.2), respectively. Note that the curve $\gamma_{t}^{v}=\left\{X_{0}(t, \theta) \in \mathbb{R}^{2} ; \theta \in S\right\}$ is recovered from $\{\kappa(s) ; s \leq t\}$ and $X_{0}(0,0)$ as

$$
\begin{aligned}
X_{0}(t, \theta)= & X_{0}(0,0)+\left(\int_{0}^{\theta} \frac{\sin \theta^{\prime}}{\kappa\left(t, \theta^{\prime}\right)} d \theta^{\prime},-\int_{0}^{\theta} \frac{\cos \theta^{\prime}}{\kappa\left(t, \theta^{\prime}\right)} d \theta^{\prime}\right) \\
& +\left(\int_{0}^{t}\left(\kappa(s, 0)-\bar{\kappa}(s)+\frac{c}{\left|\gamma_{s}\right|} v(s)\right) d s, \int_{0}^{t} \frac{\partial}{\partial \theta} \kappa(s, 0) d s\right) .
\end{aligned}
$$

Once $\gamma_{t}^{v}$ is determined, one can define the signed distance function $d^{v}(t, x)$.

We consider the time

$$
\begin{aligned}
\tau_{L}^{v}= & \inf \left\{t>0 ; m\left(\kappa_{t}\right)>L \text { or } \operatorname{dist}\left(\gamma_{t}, \partial D\right)<\frac{1}{L}\right. \text { or } \\
& \left.\inf _{x \in V_{3 \delta}^{t}}\left(I_{2}+d(t, x) \nabla^{2} d(t, x)\right)<\frac{1}{L}\right\},
\end{aligned}
$$

where $I_{2}$ is the unit matrix, $m(\kappa)=\max _{\theta \in S}\left\{\kappa(\theta), \kappa(\theta)^{-1},\left|\kappa^{(1)}(\theta)\right|\right\}, \kappa^{(n)}=$ $\partial^{n} \kappa / \partial \theta^{n}, \kappa_{t}=\kappa(t) \equiv \kappa^{v}(t)$ is the solution of (5.7) and $\gamma_{t}=\gamma_{t}^{v}$ and $d(t, x)=$ $d^{v}(t, x)$ are determined as above. We define the stopping time $\tau_{L}^{\varepsilon}:=\tau_{L}^{v}$ with $v=\alpha \dot{w}^{\varepsilon}$; in other words, $\tau_{L}^{\varepsilon}$ is the stopping time for the solution of (5.6). Note that the uniqueness of solutions implies that the solution of (5.6) coincides with that of (5.7) with $v=\alpha \dot{w}^{\varepsilon}$ for $0 \leq t \leq \tau_{L}^{\varepsilon}$. The reason that we care $I_{2}+d \nabla^{2} d$ will be clear in (0.48) in the proof of Lemma 5.1 in [14].

We are now in the position to show Assumption 3.1 assuming Assumption 1.2 in a.s.-sense.

LEMMA 5.1. For every $L \in \mathbb{N}$, there exist $N=N(K) \in \mathbb{N}$, a stopping time $T=\tau(\omega)>0$ and $C=C\left(C_{\mathcal{V}, T}, K, T, L\right)>0$ such that

$$
\begin{array}{r}
\sup _{1 \leq i \leq 2} \sup _{\theta \in S}\left|\partial_{t}^{k} \partial_{\theta}^{m} X_{0}^{i}(\cdot, \theta)\right|_{0, T} \leq C\left(1+|v|_{N, T}\right)^{N}, \\
\sup _{x \in V_{3 \delta}^{t}}\left|\partial_{t}^{k} \partial^{\mathbf{m}} \theta(\cdot, x)\right|_{0, T} \leq C\left(1+|v|_{N, T}\right)^{N},
\end{array}
$$

for every $v \in \mathcal{V}=\left\{\alpha \dot{w}^{\varepsilon} ; 0<\varepsilon \leq \varepsilon_{0}^{*}\right\}, k=0,1, \ldots, K$ and $m,|\mathbf{m}| \leq M$, where $\theta(t, x)=\mathbf{S}(t, x) \in S$ is the inverse function of $x=X_{0}(t, \theta)+r \vec{n}\left(X_{0}(t, \theta)\right)$ defined below (2.10). 
The next task is to show that Assumption 1.2 holds in the sense of law in our setting. We prepare the following theorem which gives the construction of the solution of (5.5) and, therefore, the local solution of (5.1) in the sense of law, and shows the convergence in law of the solution of (5.7) with $v(t)=\alpha \dot{w}^{\varepsilon}(t)$ to that of (5.1) locally in time. We use the usual martingale method. Since this is similar to [11], Section 5, the details are omitted; see Proposition 5.15 and Theorem 5.16 stated below. The noise $\dot{w}^{\varepsilon}(t)$ is taken the same as the second example given in Section 4.1, that is, as in (4.4).

THEOREM 5.2. For each $m \in \mathbb{N}, L>0$ and $T>0$, let $P^{\varepsilon}$ be the distribution of the solution $\kappa^{\varepsilon}(t, \cdot)$ of $(5.6)$ on $C\left([0, T], C^{m}(S)\right)$. Then $\left\{P^{\varepsilon}\right\}_{0<\varepsilon<1}$ is tight.

To prove this theorem, we need to show the following two propositions; see Theorems 6.1 and 4.2 in [11] for the first proposition and Proposition 4.1 in [11] for the second. Note that the assertion of Proposition 5.3 is a little weaker than [11], because an additional term appears in the bound given in Lemma 5.11.

PROPOSITION 5.3. There exist stopping times $\tilde{\sigma}^{\varepsilon} \equiv \tilde{\sigma}_{L}^{\varepsilon}, 0<\varepsilon<1$, such that $\tilde{\sigma}^{\varepsilon}>0$ a.s.,

$$
\lim _{\varepsilon \downarrow 0} P\left(\tilde{\sigma}^{\varepsilon}>T\right)=1
$$

for every $T>0$ and

$$
\sup _{0<\varepsilon<1} E\left[\sup _{0 \leq t \leq T}\left\|\kappa^{(n)}\left(t \wedge \tilde{\sigma}^{\varepsilon}\right)\right\|_{L^{p}(S)}^{p}\right]<\infty
$$

for every $n \in \mathbb{Z}_{+}=\{0,1,2, \ldots\}$ and $p \geq 1$, where $\kappa(t)=\kappa^{\varepsilon}(t)$ is the solution of (5.6).

Proposition 5.4. For every $\varphi \in C^{\infty}(S)$ and $p \geq 0$,

$$
E\left[\left|\left\langle\kappa^{\varepsilon}\left(t_{2}\right)-\kappa^{\varepsilon}\left(t_{1}\right), \varphi\right\rangle\right|^{p}\right] \leq C\left(t_{2}-t_{1}\right)^{p / 2}, \quad 0 \leq t_{1}<t_{2} \leq T,
$$

where $\langle\kappa, \varphi\rangle=\int_{S} \kappa(\theta) \varphi(\theta) d \theta$.

Once we have the uniform moment estimates (5.12), relying on the criterion due to Holley and Stroock (see [9]), the conclusion of Theorem 5.2 follows from the weak tightness of $\left\{\kappa^{\varepsilon}(t)\right\}_{0<\varepsilon<1}$ shown in Proposition 5.4. Indeed, (5.12) implies the tightness of $\left\{\kappa^{\varepsilon}\left(t \wedge \tilde{\sigma}^{\varepsilon}\right)\right\}_{0<\varepsilon<1}$ and this shows the conclusion from (5.11).

ProOF OF Proposition 5.4. We follow the proof of Proposition 4.1 in [11] and only sketch the proof of the proposition. The difference from [11] is that we 
need to replace $h^{\prime}(\kappa(t, \theta))$ by $D h(\theta, \kappa(t))$, the Fréchet derivative in $\kappa$. Indeed, the integral form of our equation (5.6) is

$$
\begin{aligned}
\kappa\left(t_{2}, \cdot\right)-\kappa\left(t_{1}, \cdot\right)= & \int_{t_{1}}^{t_{2}} a(\kappa(s, \cdot)) \frac{\partial^{2} \kappa(s, \cdot)}{\partial \theta^{2}} d s+\int_{t_{1}}^{t_{2}} b(\cdot, \kappa(s)) d s \\
& +\int_{t_{1}}^{t_{2}} h(\cdot, \kappa(s)) \dot{w}^{\varepsilon}(s) d s .
\end{aligned}
$$

To complete the proof, it suffices to show

$$
E\left[|X(t)|^{p}\right] \leq C\left(t-t_{1}\right)^{\frac{p}{2}}, \quad t \geq t_{1}, p \in 2 \mathbb{N},
$$

for $X(t)=\int_{t_{1}}^{t} \Phi(\kappa(s)) \dot{w}^{\varepsilon}(s) d s, t \geq t_{1}$, where $\Phi(\kappa)=\int_{S} h(\theta, \kappa) \varphi(\theta) d \theta$. Since the Fréchet derivatives toward $\psi \in L^{2}(S)$ of $h(\theta, \kappa)$ and $|\gamma|_{L}(\kappa)$ are computed as

$$
\begin{aligned}
D h(\theta, \kappa)(\psi)= & \frac{2 c \alpha \chi_{L}^{\prime}(\kappa(\theta)) \chi_{L}(\kappa(\theta))}{|\gamma|_{L}(\kappa)} \psi(\theta) \\
& +\frac{c \alpha \chi_{L}(\kappa(\theta))^{2}}{|\gamma|_{L}(\kappa)^{2}}\left\langle\frac{\chi_{L}^{\prime}(\kappa)}{\chi_{L}(\kappa)^{2}}, \psi\right\rangle, \\
D|\gamma|_{L}(\kappa)(\psi)= & -\left\langle\frac{\chi_{L}^{\prime}(\kappa)}{\chi_{L}^{2}(\kappa)}, \psi\right\rangle,
\end{aligned}
$$

respectively, we have

$$
X(t)^{p}=p \int_{t_{1}}^{t} \dot{w}^{\varepsilon}(s) d s\left[\int_{t_{1}}^{s} \Psi_{1}(r) d r+\int_{t_{1}}^{s} \Psi_{2}(r) \dot{w}^{\varepsilon}(r) d r\right],
$$

where

$$
\begin{aligned}
\Psi_{1}(r)= & X(r)^{p-1} \int_{S} \operatorname{Dh}(\theta, \kappa(r))\left(a(\kappa(r, \cdot)) \kappa^{(2)}(r, \cdot)+b(\cdot, \kappa(r))\right) \varphi(\theta) d \theta \\
\Psi_{2}(r)= & (p-1) X(r)^{p-2} \Phi(\kappa(r))^{2} \\
& +X(r)^{p-1} \int_{S} \operatorname{Dh}(\theta, \kappa(r))(h(\cdot, \kappa(r))) \varphi(\theta) d \theta .
\end{aligned}
$$

Noting that $a, b, h, \chi_{L}$ and its derivative $\chi_{L}^{\prime}$ are bounded, similar to the proof of Proposition 4.1 in [11], the conclusion is shown.

The proof of Proposition 5.3 can be completed similar to those of Theorem 6.1 and Lemmas 6.1 to 6.16 in [11]. The difference between [11] and ours is that the coefficients $b=b(\theta, \kappa)$ and $h=h(\theta, \kappa)$ of the SPDE (5.6) are functionals of $\kappa=\{\kappa(\theta) ; \theta \in S\}$ in our case. This requires some more careful computations, although most of the proof is similar to that in [11]. Another difference is that, in [11], the noise is taken as $\dot{w}^{\varepsilon}(t)=\varepsilon^{-\gamma} \xi\left(\varepsilon^{-2 \gamma} t\right), \gamma>0$ with $\xi$ introduced in Section 4.1, and hence $\left|\dot{w}^{\varepsilon}(t)\right| \leq M \varepsilon^{-\gamma}$ holds. In our case, $\dot{w}^{\varepsilon}$ is given by (4.4) 
and satisfies $\left|\dot{w}^{\varepsilon}(t)\right| \leq \frac{M}{A} \psi(\varepsilon)$, where $\psi(\varepsilon)$ is the function of $\varepsilon$ defined as (4.1). Therefore, we need to replace $\varepsilon^{-\gamma}$ appearing in lemmas of [11] by $\psi(\varepsilon)$, but we can obtain similar results to that of [11]. Here, we indicate only the different points in the proof from that in [11].

Before giving the proof of Proposition 5.3, we prepare several lemmas parallel to [11]. In the rest of this section, we assume $p \in 2 \mathbb{N}$. By directly computing from (5.6), we have

$$
\frac{d}{d t}\left\|\kappa_{t}^{(n)}\right\|_{L^{p}}^{p}=p\left(\Phi_{1}^{(n)}\left(\kappa_{t}\right)+\Phi_{2}^{(n)}\left(\kappa_{t}\right)+\Phi_{3}^{(n)}\left(\kappa_{t}\right) \dot{w}^{\varepsilon}(t)\right),
$$

where $L^{p}=L^{p}(S)$ and

$$
\begin{aligned}
& \Phi_{1}^{(n)}(\kappa)=\int_{S}\left\{\kappa^{(n)}(\theta)\right\}^{p-1}\left\{a(\kappa(\theta)) \kappa^{(2)}(\theta)\right\}^{(n)} d \theta, \\
& \Phi_{2}^{(n)}(\kappa)=\int_{S}\left\{\kappa^{(n)}(\theta)\right\}^{p-1}\{b(\theta, \kappa)\}^{(n)} d \theta, \\
& \Phi_{3}^{(n)}(\kappa)=\int_{S}\left\{\kappa^{(n)}(\theta)\right\}^{p-1}\{h(\theta, \kappa)\}^{(n)} d \theta .
\end{aligned}
$$

Then, $\frac{d}{d t} \Phi_{3}^{(n)}\left(\kappa_{t}\right)$ can be decomposed into

$$
\frac{d}{d t} \Phi_{3}^{(n)}\left(\kappa_{t}\right)=\Psi_{1}^{(n)}\left(\kappa_{t}\right)+\Psi_{2}^{(n)}\left(\kappa_{t}\right) \dot{w}^{\varepsilon}(t)
$$

where

$$
\begin{aligned}
\Psi_{1}^{(n)}(\kappa)= & (p-1) \int_{S}\left\{\kappa^{(n)}(\theta)\right\}^{p-2}\{h(\theta, \kappa)\}^{(n)}\left\{a(\kappa(\theta)) \kappa^{(2)}(\theta)+b(\theta, \kappa)\right\}^{(n)} d \theta \\
& +\int_{S}\left\{\kappa^{(n)}(\theta)\right\}^{p-1}\left[D h(\theta, \kappa)\left(a(\kappa(\cdot)) \kappa^{(2)}+b(\cdot, \kappa)\right)\right]^{(n)} d \theta, \\
\Psi_{2}^{(n)}(\kappa)= & (p-1) \int_{S}\left\{\kappa^{(n)}(\theta)\right\}^{p-2}\left[\{h(\theta, \kappa)\}^{(n)}\right]^{2} d \theta \\
& +\int_{S}\left\{\kappa^{(n)}(\theta)\right\}^{p-1}\{D h(\theta, \kappa)(h(\cdot, \kappa))\}^{(n)} d \theta .
\end{aligned}
$$

Set

$$
\psi_{p}^{(n)}(\kappa)=\int_{S}\left\{\kappa^{(n)}(\theta)\right\}^{p-2}\left\{\kappa^{(n+1)}(\theta)\right\}^{2} d \theta, \quad p \geq 2, n \geq 1 .
$$

We denote by $\mathcal{P}_{n}$ the family of polynomials of the forms $P\left(y_{1}, \ldots, y_{n-1}, z ; \kappa\right)=$ $\sum_{\alpha} g_{1, \alpha}(\kappa) g_{2, \alpha}(z) y^{\alpha}, y_{i} \in \mathbb{R}, 1 \leq i \leq n-1, \kappa \in \mathbb{R}, z \in L^{2}(S)$ with $g_{1, \alpha} \in C_{b}^{\infty}(\mathbb{R})$, $g_{2, \alpha} \in C_{b}^{\infty}\left(L^{2}(S)\right), \alpha=\left(\alpha_{1}, \ldots, \alpha_{n-1}\right) \in \mathbb{Z}_{+}^{n-1}, y^{\alpha}=y_{1}^{\alpha_{1}} \cdots y_{n-1}^{\alpha_{n-1}}$ and the sum $\sum_{\alpha}$ finite, where $C_{b}^{\infty}\left(L^{2}(S)\right)$ stands for the family of infinitely Fréchet differentiable functions on $L^{2}(S)$ having bounded derivatives.

The term $\Phi_{1}^{(n)}$ coincides with that appearing in [11], Lemma 6.1, so that we have the following lemma. 
LEMmA 5.5 (Estimate for $\Phi_{1}^{(n)}$ ). For $n \geq 1$, there exist constants $c=$ $c(n, p, L)$ and $C=C(n, p, L)>0$ such that

$$
\Phi_{1}^{(n)}(\kappa) \leq-c \psi_{p}^{(n)}(\kappa)+C\left\{\left\|\kappa^{(n)}\right\|_{L^{p}}^{p}+\sum_{i=1}^{3}\left\|P_{i}\right\|_{L^{p}}^{p}\right\},
$$

for some $P_{i}=P_{i}\left(\kappa^{(1)}(\theta), \ldots, \kappa^{(n-1)}(\theta), \kappa ; \kappa(\theta)\right) \in \mathcal{P}_{n}$ with $g_{2, \alpha, i} \equiv 1, i=1,2,3$, that is, $P_{i}$ of the forms $P_{i}\left(y_{1}, \ldots, y_{n-1}, z ; \kappa\right)=\sum_{\alpha} g_{1, \alpha, i}(\kappa) y^{\alpha}$ with $g_{1, \alpha, i} \in$ $C_{b}^{\infty}(\mathbb{R}), i=1,2,3$. In particular, in the case $n=1,(5.18)$ holds with $C=0$ and $P_{1}=P_{2}=P_{3}=0$.

As for the terms $\Phi_{2}^{(n)}$ and $\Phi_{3}^{(n)}$, we have the upper bounds for them as in the next lemma.

LEMMA 5.6 (Estimates for $\Phi_{2}^{(n)}$ and $\Phi_{3}^{(n)}$ ). For $n \geq 1$, there exists a constant $C=C(n, p, L)>0$ such that

$$
\begin{aligned}
& \left|\Phi_{2}^{(n)}(\kappa)\right| \leq C\left\{\left\|\kappa^{(n)}\right\|_{L^{p}}^{p}+\left\|P_{4}\right\|_{L^{p}}^{p}\right\}, \\
& \left|\Phi_{3}^{(n)}(\kappa)\right| \leq C\left\{\left\|\kappa^{(n)}\right\|_{L^{p}}^{p}+\left\|P_{5}\right\|_{L^{p}}^{p}\right\},
\end{aligned}
$$

for some $P_{i}=P_{i}\left(\kappa^{(1)}(\theta), \ldots, \kappa^{(n-1)}(\theta), \kappa ; \kappa(\theta)\right) \in \mathcal{P}_{n}, i=4$, 5. In particular, in the case $n=1$, these estimates hold with $P_{4}=P_{5}=0$.

We have another estimate for $\Phi_{3}^{(n)}(\kappa)$. In what follows, $\psi(\varepsilon)$ denotes the function given in (4.1).

LEMMA 5.7. For every $n \geq 1$ and $\delta>0$,

$$
\left|\Phi_{3}^{(n)}(\kappa)\right| \leq \psi(\varepsilon)^{-1}(p-1)\left[\delta \psi_{p}^{(n)}(\kappa)+\delta^{-1}\left\|\kappa^{(n)}\right\|_{L^{p}}^{p}+\delta^{-1} \psi(\varepsilon)^{p}\left\|P_{6}\right\|_{L^{p}}^{p}\right],
$$

for some $P_{6}=P_{6}\left(\kappa^{(1)}(\theta), \ldots, \kappa^{(n-1)}(\theta), \kappa ; \kappa(\theta)\right) \in \mathcal{P}_{n}$.

Next, we give a bound for $\Psi_{2}^{(n)}$ for $n \geq 1$.

LEMmA 5.8 (Estimate for $\Psi_{2}^{(n)}$ ). For $n \geq 1$, there exists a constant $C=$ $C(n, p, L)>0$ such that

$$
\left|\Psi_{2}^{(n)}(\kappa)\right| \leq C\left[\left\|\kappa^{(n)}\right\|_{L^{p}}^{p}+\sum_{i=7}^{8}\left\|P_{i}\right\|_{L^{p}}^{p}\right],
$$

for some $P_{i}=P_{i}\left(\kappa^{(1)}(\theta), \ldots, \kappa^{(n-1)}(\theta), \kappa ; \kappa(\theta)\right) \in \mathcal{P}_{n}, i=7,8$.

Let us give an estimate for $\Psi_{1}^{(n)}$. We start with the case $n=1$. 
LEMMA 5.9. For every $\delta>0$, we have

$$
\left|\Psi_{1}^{(1)}(\kappa)\right| \leq C\left[1+\left\|\kappa^{(1)}\right\|_{L^{p}}^{p}+\psi(\varepsilon)^{-1} \delta^{-1}\left\|\kappa^{(1)}\right\|_{L^{p+2}}^{p+2}+(1+\psi(\varepsilon) \delta) \psi_{p}^{(1)}(\kappa)\right],
$$

for some $C=C(1, p, L)>0$.

Next, we consider the the case $n \geq 2$. For $n \geq 2$, by the integration by parts formula for $\Psi_{1}^{(n)}$, it can be decomposed into

$$
\Psi_{1}^{(n)}(\kappa)=-(p-1)(p-2) \Psi_{a}^{(n)}(\kappa)-(p-1) \Psi_{b}^{(n)}(\kappa)-(p-1) \Psi_{c}^{(n)}(\kappa),
$$

where

$$
\begin{aligned}
& \Psi_{a}^{(n)}(\kappa)=\int_{S}\left\{\kappa^{(n)}(\theta)\right\}^{p-3} \kappa^{(n+1)}(\theta)\{h(\theta, \kappa)\}^{(n)}\{A(\theta, \kappa)\}^{(n-1)} d \theta, \\
& \Psi_{b}^{(n)}(\kappa)=\int_{S}\left\{\kappa^{(n)}(\theta)\right\}^{p-2}\{h(\theta, \kappa)\}^{(n+1)}\{A(\theta, \kappa)\}^{(n-1)} d \theta, \\
& \Psi_{c}^{(n)}(\kappa)=\int_{S}\left\{\kappa^{(n)}(\theta)\right\}^{p-2} \kappa^{(n+1)}(\theta)[D h(\theta, \kappa)(A(\cdot, \kappa))]^{(n-1)} d \theta .
\end{aligned}
$$

Then we have the following three lemmas for $\Psi_{b}^{(n)}, \Psi_{c}^{(n)}$ and $\Psi_{a}^{(n)}$.

LEMMA 5.10 (Estimate on $\Psi_{b}^{(n)}$ ). For $n \geq 2$, there exists a constant $C=$ $C(n, p, L)>0$ such that

$$
\left|\Psi_{b}^{(n)}(\kappa)\right| \leq C\left[\psi_{p}^{(n)}(\kappa)+\left\|\kappa^{(n)}\right\|_{L^{p}}^{p}+\sum_{i=9}^{12}\left\|P_{i}\right\|_{L^{p}}^{p}\right],
$$

for some $P_{i}=P_{i}\left(\kappa^{(1)}(\theta), \ldots, \kappa^{(n-1)}(\theta), \kappa ; \kappa(\theta)\right) \in \mathcal{P}_{n}, i=9, \ldots, 12$.

The following estimate is similar to [11], Lemma 6.7, but we need the second term additionally for this estimate.

LEMMA 5.11 (Estimate on $\left.\Psi_{c}^{(n)}\right)$. For $n \geq 2$, there exists a constant $C=$ $C(n, p, L)>0$ such that

$$
\begin{aligned}
\left|\Psi_{c}^{(n)}(\kappa)\right| \leq & C\left[\psi_{p}^{(n)}(\kappa)+\left\|\kappa^{(n)}\right\|_{L^{p}}^{p}+\sum_{i=13}^{15}\left\|P_{i}\right\|_{L^{p}}^{p}\right] \\
& +C\left\|\kappa^{(1)}\right\|_{L^{2}}^{4}\left(\left\|\kappa^{(n)}\right\|_{L^{p}}^{p}+\|Q\|_{L^{p}}^{p}\right),
\end{aligned}
$$

for some $P_{i}=P_{i}\left(\kappa^{(1)}(\theta), \ldots, \kappa^{(n-1)}(\theta), \kappa ; \kappa(\theta)\right) \in \mathcal{P}_{n}, i=13,14,15$ and $Q=$ $Q\left(\kappa^{(1)}(\theta), \ldots, \kappa^{(n-1)}(\theta), \kappa ; \kappa(\theta)\right) \in \mathcal{P}_{n}$ with $g_{2, \alpha} \equiv 1$, namely, $Q$ does not contain any functional of $\kappa$. 
Recalling again that $\partial_{\kappa(\theta)}^{l} h(\theta, \kappa)$ and $\partial_{\kappa(\theta)}^{l} b(\theta, \kappa), l \in \mathbb{Z}_{+}$, are all bounded and following the proof of [11], Lemma 6.9, we have the next lemma.

LEMMA 5.12 (Estimate on $\left.\Psi_{a}^{(n)}\right)$. For every $n \geq 2$ and $\beta^{\prime} \in(0,1)$, there exist constants $C, N, q>0$ such that

$$
\begin{aligned}
\left|\Psi_{a}^{(n)}(\kappa)\right| \leq & C\left[\psi_{p}^{(n)}(\kappa)+\left\|\kappa^{(n)}\right\|_{L^{p}}^{p}+\sum_{i=16}^{19}\left\|P_{i}\right\|_{L^{p}}^{p}\right] \\
& +C\left[1+\sum_{i=1}^{n-1}\left\|\kappa^{(i)}\right\|_{L^{p}}^{N}+\left\|\kappa^{(n)}\right\|_{L^{q}}^{\beta^{\prime}}\right] \psi_{2}^{(n)}(\kappa),
\end{aligned}
$$

for some $P_{i}=P_{i}\left(\kappa^{(1)}(\theta), \ldots, \kappa^{(n-1)}(\theta), \kappa ; \kappa(\theta)\right) \in \mathcal{P}_{n}, i=16, \ldots, 19$.

Now we estimate the conditional expectation of the integral of the third term on the right-hand side of (5.15). Let us recall $\mathcal{F}_{s, t}=\sigma(\xi(u), u \in[s, t])$, where $\xi$ is the stochastic process given in Section 4.1. Set $\mathcal{F}_{s, t}^{\varepsilon}=\mathcal{F}_{\psi(\varepsilon)^{2} s, \psi(\varepsilon)^{2} t}$. Recall also that in our case $\dot{w}^{\varepsilon}$ is given by (4.4) and $\left|\dot{w}^{\varepsilon}(t)\right| \leq \frac{M}{A} \psi(\varepsilon)$ holds. By replacing the divergent factor $\varepsilon^{-\gamma}$ in [11], Lemmas 6.10 and 6.11 by $\psi(\varepsilon)$, we obtain the following two lemmas. The proofs are the same and, therefore, omitted.

LEMMA 5.13. For $0 \leq s \leq t \leq T$, we have

$$
\begin{aligned}
& \left|E\left[\int_{s}^{t} \Phi_{3}^{(n)}\left(\kappa_{r}\right) \dot{w}^{\varepsilon}(r) d r \mid \mathcal{F}_{0, s}^{\varepsilon}\right]\right| \\
& \quad \leq C \psi(\varepsilon)^{-1}\left|\Phi_{3}^{(n)}\left(\kappa_{s}\right)\right|+C \int_{s}^{t} E\left[\psi(\varepsilon)^{-1}\left|\Psi_{1}^{(n)}\left(\kappa_{r}\right)\right|+\left|\Psi_{2}^{(n)}\left(\kappa_{r}\right)\right| \mid \mathcal{F}_{0, s}^{\varepsilon}\right] d r,
\end{aligned}
$$

for some $C=C(M, A)>0$.

LEMMA 5.14 (Rough estimates uniform in $\omega$ ). Assume $\kappa_{0}=\kappa(0) \in C^{\infty}(S)$ is independent of $\varepsilon$. Then there exist constants $C=C(T, n, p)$ and $N=N(n, p)>0$ such that

$$
\begin{aligned}
\left\|\kappa_{t}^{(n)}\right\|_{L^{p}} & \leq C \psi(\varepsilon)^{N}, 0 \leq t \leq T \\
\int_{0}^{T} \psi_{p}^{(n)}\left(\kappa_{t}\right) d t & \leq C \psi(\varepsilon)^{N} \\
\left\|\kappa_{t}^{(n)}\right\|_{L^{p}}^{p} & \leq\left[\left\|\kappa_{s}^{(n)}\right\|_{L^{p}}^{p}+C \psi(\varepsilon) \int_{s}^{t} \sum_{i=1}^{5}\left\|P_{i}\left(\kappa_{r}\right)\right\|_{L^{p}}^{p} d r\right] e^{C \psi(\varepsilon)(t-s)},
\end{aligned}
$$


for $0 \leq s \leq t \leq T$. In particular, if $n=1$, we can take $N=1$ in (5.19) and $N=p$ in (5.20), that is,

$$
\begin{aligned}
\left\|\kappa_{t}^{(1)}\right\|_{L^{p}}^{p} \leq C \psi(\varepsilon), \quad 0 \leq t \leq T, \\
\int_{0}^{T} \psi_{p}^{(1)}\left(\kappa_{t}\right) d t \leq C \psi(\varepsilon)^{p} .
\end{aligned}
$$

Under the preparation of Lemmas 5.5 to 5.14 , one can complete the proof of Proposition 5.3.

PROOF OF PROPOSITION 5.3. The proof of (5.12) is completed by induction in the following three steps as in [11], Section 6.7. Step 1: we prove (5.12) for $n=1$. Step 2: we prove (5.12) for $n$ assuming that it holds for $i=1, \ldots, n-1$. Step 3: we prove (5.12) for $n=0$.

As for Step 1, using the estimates on $\Phi_{1}^{(1)}, \Phi_{2}^{(1)}, \Phi_{3}^{(1)}, \Psi_{1}^{(1)}$ and $\Psi_{2}^{(1)}$, a similar method to [11] shows

$$
\sup _{0<\varepsilon<1} E\left[\sup _{t \in[0, T]}\left\|\kappa_{t}^{(1)}\right\|_{L^{p}}^{p}\right]<\infty .
$$

In Step 2, integrating the both sides of (5.15) from $s$ to $t$ and taking the conditional expectation, and then, using Lemmas 5.5 to 5.14 and noting that $\left|\dot{w}^{\varepsilon}(t)\right|$ satisfies $\left|\dot{w}^{\varepsilon}(t)\right| \leq \frac{M}{A} \psi(\varepsilon)$, we have

$$
\begin{aligned}
& E\left[\left\|\kappa_{t}^{(n)}\right\|_{L^{p}}^{p} \mid \mathcal{F}_{0, s}^{\varepsilon}\right]+c^{\prime \prime} \int_{s}^{t} E\left[\psi_{p}^{(n)}\left(\kappa_{r}\right) \mid \mathcal{F}_{0, s}^{\varepsilon}\right] d r \\
& \leq\left(1+C \psi(\varepsilon)^{-1}\right)\left\|\kappa_{s}^{(n)}\right\|_{L^{p}}^{p}+C \psi(\varepsilon)^{-1}\left\|P_{5}\left(\kappa_{s}\right)\right\|_{L^{p}}^{p} \\
&+C \int_{s}^{t} E\left[\left\|\kappa_{r}^{(n)}\right\|_{L^{p}}^{p} \mid \mathcal{F}_{0, s}^{\varepsilon}\right] d r+C \int_{s}^{t} E\left[\mathcal{P}\left(\kappa_{r}\right) \mid \mathcal{F}_{0, s}^{\varepsilon}\right] d r \\
&+C \psi(\varepsilon)^{-1} \int_{s}^{t} E\left[\left\|\kappa_{r}^{(1)}\right\|_{L^{2}}^{4}\left\|\kappa_{r}^{(n)}\right\|_{L^{p}}^{p} \mid \mathcal{F}_{0, s}^{\varepsilon}\right] d r \\
&+C \psi(\varepsilon)^{-1} \int_{s}^{t} E\left[\left\|\kappa_{r}^{(1)}\right\|_{L^{2}}^{4}\left\|Q\left(\kappa_{r}\right)\right\|_{L^{p}}^{p} \mid \mathcal{F}_{0, s}^{\varepsilon}\right] d r \\
&+C \psi(\varepsilon)^{-1}(p-2) \\
& \times \int_{s}^{t} E\left[\left\{1+\psi(\varepsilon)^{\delta}+\sum_{i=1}^{n-1}\left\|\kappa_{r}^{(i)}\right\|_{L^{q}}^{N}\right\} \psi_{2}^{(n)}\left(\kappa_{r}\right) \mid \mathcal{F}_{0, s}^{\varepsilon}\right] d r
\end{aligned}
$$

for every $\varepsilon \in\left(0, \varepsilon_{0}\right)$ with a sufficiently small $\varepsilon_{0}$ in such a way that $\frac{C}{c} \psi\left(\varepsilon_{0}\right)^{-1} \ll$ 1 (hence, there exists some $c^{\prime \prime}>0$ ), for every $\delta>0$ and some $N=N(\delta), q=$ $q(\delta) \geq 1$, where $\mathcal{P}\left(\kappa_{t}\right)=\sum_{1 \leq i \leq 19, i \neq 5,6}\left\|P_{i}\left(\kappa_{t}\right)\right\|_{L^{p}}^{p}$. Let us define $\tilde{\sigma}_{\varepsilon}=\inf \{t>$ $\left.s ;\left\|\kappa_{t}^{(1)}\right\|_{L^{2}}>\psi(\varepsilon)^{\frac{1}{4}}\right\}$ and $\tilde{\sigma}_{\varepsilon}=\infty$ if the set $\{\cdot\}$ is empty. Then the estimate (5.21) 
implies (5.11). Note that (5.22) is also true with $t$ replaced by $t \wedge \tilde{\sigma}_{\varepsilon}$. Thus, by similar manner to [11], Section 6.7 with $\varepsilon^{-\gamma}$ replaced by $\psi(\varepsilon)$, we have

$$
\sup _{0<\varepsilon<1} E\left[\sup _{t \in[0, T]}\left\|\kappa_{t \wedge \tilde{\sigma}_{\varepsilon}}^{(n)}\right\|_{L^{p}}^{p}\right]<\infty ;
$$

see [11], Lemmas 6.12 to 6.16 . Finally, Step 3 can be completed by using again [11], Section 6.7 with $\varepsilon^{-\gamma}$ replaced by $\psi(\varepsilon)$ for the estimates on $\Phi_{1}^{(0)}, \Phi_{2}^{(0)}, \Phi_{3}^{(0)}$, $\Psi_{1}^{(0)}$ and $\Psi_{2}^{(0)}$. Indeed, we obtain

$$
\sup _{0<\varepsilon<1} E\left[\sup _{t \in[0, T]}\left\|\kappa_{t}\right\|_{L^{p}}^{p}\right]<\infty .
$$

As a result, we get (5.12) for every $n \in \mathbb{Z}_{+}$and $p \in 2 \mathbb{N}$, so that for every $p \geq 1$.

We now show the pathwise uniqueness for (5.5). For this, we apply the energy inequality; cf. Lemma 5.2 in [11]. To do this, we need to rewrite (5.5) into Itô's form. The Fréchet derivatives toward $\psi \in L^{2}(S)$ of $h(\theta, \kappa)$ and $|\gamma|_{L}(\kappa)$ are computed as in (5.13) and (5.14), respectively. Thus, we have that

$$
\begin{aligned}
h(\theta, \kappa(t)) \circ d w_{t} & =h(\theta, \kappa(t)) d w_{t}+\frac{1}{2} d\{h(\theta, \kappa(t))\} d w_{t} \\
& =h(\theta, \kappa(t)) d w_{t}+\frac{1}{2} \operatorname{Dh}(\theta, \kappa(t))\left(h\left(\theta_{1}, \kappa(t)\right)\right) d t \\
& =h(\theta, \kappa(t)) d w_{t}+g(\theta, \kappa(t)) d t,
\end{aligned}
$$

where

$$
\begin{aligned}
g(\theta, \kappa)= & \frac{c \alpha}{|\gamma|_{L}(\kappa)} \chi_{L}(\kappa(\theta)) \chi_{L}^{\prime}(\kappa(\theta)) h(\theta, \kappa) \\
& +\frac{c \alpha}{2|\gamma|_{L}(\kappa)^{2}} \chi_{L}^{2}(\kappa(\theta)) \int_{S} \frac{\chi_{L}^{\prime}\left(\kappa\left(\theta_{1}\right)\right) h\left(\theta_{1}, \kappa\right)}{\chi_{L}^{2}\left(\kappa\left(\theta_{1}\right)\right)} d \theta_{1} .
\end{aligned}
$$

Therefore, (5.5) can be rewritten into Itô's form:

$$
\frac{\partial \kappa}{\partial t}=a(\kappa) \frac{\partial^{2} \kappa}{\partial \theta^{2}}+\tilde{b}(\cdot, \kappa)+h(\cdot, \kappa) \dot{w}_{t}, \quad t>0, \theta \in S,
$$

where

$$
\tilde{b}(\cdot, \kappa)=b(\cdot, \kappa)+g(\cdot, \kappa)
$$

Proposition 5.15 (cf. Lemma 5.2 in [11]). Let $\kappa_{i}(t), i=1,2$, be two solutions of (5.5) or equivalently (5.23) satisfying $\kappa_{i}(t) \in C\left([0, T], C^{2}(S)\right)$ a.s. and having the same initial data: $\kappa_{1}(0)=\kappa_{2}(0) \in C^{2}(S)$. Then we have $P\left(\kappa_{1}(t)=\right.$ $\kappa_{2}(t)$ for all $\left.t \in[0, T]\right)=1$. 
PROOF. By applying Itô's formula,

$$
\left\|\kappa_{1}(t)-\kappa_{2}(t)\right\|_{L^{2}}^{2}=\int_{0}^{t}\left\{I_{1}+I_{2}\right\} d s+\int_{0}^{t} I_{3} d w_{s},
$$

where

$$
\begin{aligned}
& I_{1}=2 \int\left(\kappa_{1}-\kappa_{2}\right)\left\{a\left(\kappa_{1}\right) \kappa_{1}^{(2)}-a\left(\kappa_{2}\right) \kappa_{2}^{(2)}\right\}, \\
& I_{2}=2 \int\left(\kappa_{1}-\kappa_{2}\right)\left\{\tilde{b}\left(\cdot, \kappa_{1}\right)-\tilde{b}\left(\cdot, \kappa_{2}\right)\right\}+\left\|h\left(\cdot, \kappa_{1}\right)-h\left(\cdot, \kappa_{2}\right)\right\|_{L^{2}}^{2}, \\
& I_{3}=2 \int\left(\kappa_{1}-\kappa_{2}\right)\left\{h\left(\cdot, \kappa_{1}\right)-h\left(\cdot, \kappa_{2}\right)\right\},
\end{aligned}
$$

and the norm $\|\cdot\|_{L^{p}(S)}$ will be simply denoted by $\|\cdot\|_{L^{p}}$ for $p \in[1, \infty]$. Noting that $a(\kappa) \geq a_{0}=1 / 2 N>0$, the estimate on $I_{1}$ is exactly the same as in [11]:

$$
\begin{aligned}
I_{1} \leq & -2 a_{0}\left\|\kappa_{1}^{\prime}-\kappa_{2}^{\prime}\right\|_{L^{2}}^{2}+2\left\|a^{\prime}\right\|_{L^{\infty}}\left\|\kappa_{1}^{\prime}\right\|_{L^{\infty}}\left\{\delta^{-1}\left\|\kappa_{1}-\kappa_{2}\right\|_{L^{2}}^{2}+\delta\left\|\kappa_{1}^{\prime}-\kappa_{2}^{\prime}\right\|_{L^{2}}^{2}\right\} \\
& +2\left\|a^{\prime}\right\|_{L^{\infty}}\left\|\kappa_{2}^{(2)}\right\|_{L^{\infty}}\left\|\kappa_{1}-\kappa_{2}\right\|_{L^{2}}^{2},
\end{aligned}
$$

for every $\delta>0$. Since $D\left\{1 /|\gamma|_{L}\right\}\left(\theta_{1}, \kappa\right)=-\frac{1}{|\gamma|_{L}^{2}} D|\gamma|_{L}\left(\theta_{1}, \kappa\right),(5.14)$ shows that $\left\|D\left\{1 /|\gamma|_{L}\right\}(\cdot, \kappa)\right\|_{L^{2}}$ is bounded in $\kappa$, and thus the map $\kappa \in L^{2} \mapsto 1 /|\gamma|_{L}(\kappa) \in \mathbb{R}$ is Lipschitz continuous. This implies that

$$
I_{2} \leq C\left\|\kappa_{1}-\kappa_{2}\right\|_{L^{2}}^{2} .
$$

Now introduce a stopping time

$$
\tau_{M}=\inf \left\{t>0 ; \max \left\{\left\|\kappa_{1}\right\|_{L^{\infty}},\left\|\kappa_{2}\right\|_{L^{\infty}},\left\|\kappa_{1}^{(1)}\right\|_{L^{\infty}},\left\|\kappa_{2}^{(2)}\right\|_{L^{\infty}}\right\}>M\right\}, \quad M>0 .
$$

Then, by choosing $\delta>0$ sufficiently small, we have

$$
\begin{aligned}
\left\|\kappa_{1}\left(t \wedge \tau_{M}\right)-\kappa_{2}\left(t \wedge \tau_{M}\right)\right\|_{L^{2}}^{2} \leq & C_{M} \int_{0}^{t}\left\|\kappa_{1}\left(s \wedge \tau_{M}\right)-\kappa_{2}\left(s \wedge \tau_{M}\right)\right\|_{L^{2}}^{2} d s \\
& +\int_{0}^{t \wedge \tau_{M}} I_{3} d w_{s},
\end{aligned}
$$

for $t>0$, for some $C_{M}>0$. Therefore, taking the expectation of both sides and applying the Gronwall's lemma, we obtain

$$
E\left[\left\|\kappa_{1}\left(t \wedge \tau_{M}\right)-\kappa_{2}\left(t \wedge \tau_{M}\right)\right\|_{L^{2}}^{2}\right]=0
$$

for all $M>0$. This completes the proof of the proposition.

The pathwise uniqueness combined with the existence of the solution in the sense of law implies the existence of a strong solution. This is the well-known Yamada-Watanabe's theorem which is extended into an infinite-dimensional setting by [23]; see also [25]. As a result, we obtain the following theorem. 
THEOREM 5.16. Let $D$ be a two-dimensional bounded domain and $\gamma_{0}$ be a closed convex curve given such that $\gamma \Subset D$. Let $\kappa_{0}(\theta), \theta \in S$ be the curvature of $\gamma_{0}$. Then there exists a unique local solution $\kappa=\kappa(t, \theta)$ defined for $0 \leq t<\sigma$ and $\theta \in S$ of the SPDE (5.1), where $\sigma>0$ (a.s.) is a stopping time. In particular, the dynamics (1.4) has a unique solution for $0 \leq t<\sigma$.

Theorem 5.2 together with the remarks made before it combined with Theorem 5.16, which implies the uniqueness in law, shows that Assumption 1.2 holds in the sense of law in the setting of this section.

Acknowledgments. The authors thank Danielle Hilhorst for leading them to the problem discussed in this article and stimulating discussions. They thank two anonymous referees for their useful comments which helped to improve the paper.

\section{SUPPLEMENTARY MATERIAL}

Supplement to "Sharp interface limit for stochastically perturbed mass conserving Allen-Cahn equation"'(DOI: 10.1214/18-AOP1268SUPP; .pdf). The supplementary file provides proofs of all the lemmas, with some exceptions, stated in Sections 3-5 of the present paper.

\section{REFERENCES}

[1] Bellettini, G. (2013). Lecture Notes on Mean Curvature Flow, Barriers and Singular Perturbations. Appunti. Scuola Normale Superiore di Pisa (Nuova Serie) [Lecture Notes. Scuola Normale Superiore di Pisa (New Series)] 12. Edizioni della Normale, Pisa. MR3155251

[2] Chen, X. (1994). Spectrum for the Allen-Cahn, Cahn-Hilliard, and phase-field equations for generic interfaces. Comm. Partial Differential Equations 19 1371-1395. MR1284813

[3] Chen, X., Hilhorst, D. and LogaK, E. (2010). Mass conserving Allen-Cahn equation and volume preserving mean curvature flow. Interfaces Free Bound. 12 527-549. MR2754215

[4] De Mottoni, P. and Schatzman, M. (1995). Geometrical evolution of developed interfaces. Trans. Amer. Math. Soc. 347 1533-1589. MR1672406

[5] Elliott, C. M. and GARCKe, H. (1997). Existence results for diffusive surface motion laws. Adv. Math. Sci. Appl. 7 467-490. MR1454678

[6] Escher, J. and Simonett, G. (1998). The volume preserving mean curvature flow near spheres. Proc. Amer. Math. Soc. 126 2789-2796. MR1485470

[7] FifE, P. C. and HSIAO, L. (1988). The generation and propagation of internal layers. Nonlinear Anal. 12 19-41. MR0924750

[8] Friedman, A. (1964). Partial Differential Equations of Parabolic Type. Prentice-Hall, Inc., Englewood Cliffs, NJ. MR0181836

[9] FUnAKI, T. (1992). A stochastic partial differential equation with values in a manifold. J. Funct. Anal. 109 257-288. MR1186323

[10] FUnAKI, T. (1995). The scaling limit for a stochastic PDE and the separation of phases. Probab. Theory Related Fields 102 221-288. MR1337253

[11] FUNAKI, T. (1999). Singular limit for stochastic reaction-diffusion equation and generation of random interfaces. Acta Math. Sin. (Engl. Ser.) 15 407-438. MR1736690 
[12] FunAKI, T. (2003). Stochastic models for phase separation and evolution equations of interfaces [translation of Sūgaku 50 (1998), no. 1, 68-85]. Sugaku Expositions 16 97-116. MR1980020

[13] FUnAKI, T. (2016). Lectures on Random Interfaces. Springer, Singapore. MR3587372

[14] Funaki, T. and Yokoyama, S. (2019). Supplement to "Sharp interface limit for stochastically perturbed mass conserving Allen-Cahn equation.” DOI:10.1214/18AOP1268SUPP.

[15] GÄRTNER, J. (1983). Bistable reaction-diffusion equations and excitable media. Math. Nachr. 112 125-152. MR0726855

[16] GurTin, M. E. (1993). Thermomechanics of Evolving Phase Boundaries in the Plane. The Clarendon Press, New York. MR1402243

[17] Henry, D. (1981). Geometric Theory of Semilinear Parabolic Equations. Lecture Notes in Math. 840. Springer, Berlin. MR0610244

[18] Hilhorst, D., Matano, H., Nguyen, T. N. and Weber, H. (2016). On the large time behavior of the solutions of a nonlocal ordinary differential equation with mass conservation. J. Dynam. Differential Equations 28 707-731. MR3537353

[19] Huisken, G. (1987). The volume preserving mean curvature flow. J. Reine Angew. Math. 382 35-48. MR0921165

[20] LadyŽenskaja, O. A., Solonnikov, V. A. and Ural'Ceva, N. N. (1968). Linear and Quasilinear Equations of Parabolic Type. Translations of Mathematical Monographs 23. Amer. Math. Soc., Providence, RI. Translated from the Russian by S. Smith. MR0241822

[21] Lieberman, G. M. (1996). Second Order Parabolic Differential Equations. World Scientific Co., Inc., River Edge, NJ. MR1465184

[22] Lions, P.-L. and Souganidis, P. E. (1998). Fully nonlinear stochastic partial differential equations: Non-smooth equations and applications. C. R. Acad. Sci. Paris Sér. I Math. 327 735-741. MR1659958

[23] OndREJÁt, M. (2004). Uniqueness for stochastic evolution equations in Banach spaces. Dissertationes Math. (Rozprawy Mat.) 426 63. MR2067962

[24] Rubinstein, J. and Sternberg, P. (1992). Nonlocal reaction-diffusion equations and nucleation. IMA J. Appl. Math. 48 249-264. MR1167735

[25] TAPPE, S. (2013). The Yamada-Watanabe theorem for mild solutions to stochastic partial differential equations. Electron. Commun. Probab. 18 no. 24, 13. MR3044472

[26] Weber, H. (2010). On the short time asymptotic of the stochastic Allen-Cahn equation. Ann. Inst. Henri Poincaré Probab. Stat. 46 965-975. MR2744880

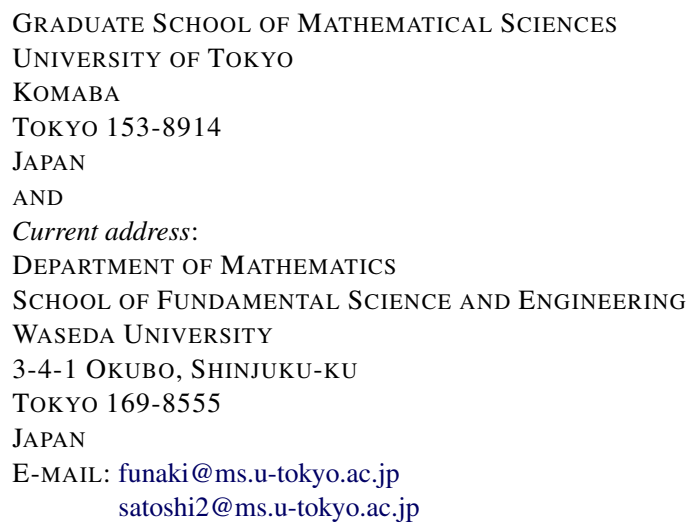

